\title{
Shifting attention to accuracy can reduce misinformation online
}

\author{
Gordon Pennycook ${ }^{1 * \dagger}$, Ziv Epstein ${ }^{2,3 *}$, Mohsen Mosleh ${ }^{3,4 *}$, \\ Antonio A. Arechar ${ }^{3,5}$, Dean Eckles ${ }^{3,6}$ \& David G. Rand ${ }^{3,6,7 \dagger}$ \\ ${ }^{1}$ Hill/Levene Schools of Business, University of Regina, ${ }^{2}$ Media Lab, Massachusetts Institute of Technology, \\ ${ }^{3}$ Sloan School of Management, Massachusetts Institute of Technology, ${ }^{4}$ SITE (Science, Innovation, \\ Technology, and Entrepreneurship) Department, University of Exeter Business School, ${ }^{5}$ Center for Research \\ and Teaching in Economics ${ }^{6}$ Institute for Data, Systems, and Society, Massachusetts Institute of Technology, \\ ${ }^{7}$ Department of Brain and Cognitive Sciences, Massachusetts Institute of Technology \\ ${ }^{\dagger}$ Corresponding authors: gordon.pennycook@uregina.ca, drand@mit.edu \\ *These authors contributed equally.
}

In recent years, there has been a great deal of concern about the proliferation of false and misleading news on social media ${ }^{1-4}$. Academics and practitioners alike have asked why people share such misinformation, and sought solutions to reduce misinformation sharing ${ }^{5-}$ 7. Here, we shed light on both of these questions. First, we find that headline veracity has little impact on sharing intentions, despite having a large impact on accuracy judgments. This dissociation suggests that sharing does not necessarily imply belief. Nonetheless, most participants say it is important to only share accurate news. To shed light on this apparent contradiction, four survey experiments and a field experiment on Twitter show that subtly shifting attention to accuracy increases the quality of news that people subsequently share. Together with additional computational analyses, these findings indicate that people often share misinformation because their attention is focused on factors other than accuracy - and thus they fail to implement a strongly-held preference for accurate sharing. Our results challenge the popular claim that people value partisanship over accuracy $^{8,9}$, and provide evidence for scalable attention-based interventions that social media platforms could easily implement to fight misinformation online. 
The sharing of misinformation on social media - including, but not limited to, blatantly false political "fake news" and misleading hyperpartisan content - has become a major focus of public debate and academic study in recent years ${ }^{1,4}$. Although misinformation is nothing new, the topic gained prominence in 2016 following the U.S. Presidential Election and the U.K.'s Brexit referendum during which entirely fabricated stories (presented as legitimate news) received wide distribution via social media - a problem that continued during the COVID-19 pandemic ${ }^{2,7}$.

Misinformation is problematic because it leads to inaccurate beliefs and can exacerbate partisan disagreement over even basic facts. Merely reading false news posts - including political posts that are extremely implausible and inconsistent with one's political ideology - makes them subsequently seem more true ${ }^{10}$. In addition to being concerning, the widespread sharing of misinformation on social media is also surprising, given the outlandishness of much of this content.

Here we test three competing theories of why people share misinformation, based respectively on confusion about what is (in)accurate, preferences for factors such as partisanship over accuracy, and inattention to accuracy.

\section{Disconnect between sharing and accuracy}

We begin with the confusion-based account, whereby people share misinformation because they mistakenly believe that it is accurate (e.g., due to media or digital illiteracy,11-14 or politically motivated reasoning ${ }^{8,9,15,16}$ ). To gain initial insight into whether mistaken beliefs are sufficient to explain the sharing of misinformation, Study 1 tests for a dissociation between what people deem to be accurate and what they would share on social media. We recruited $N=1,015$ Americans using Amazon Mechanical Turk (MTurk) ${ }^{17}$ and presented them with the headline, lede sentence, and image for 36 actual news stories taken from social media. Half of the headlines were entirely false and half were true; half of the headlines were chosen (via pretest ${ }^{18,19}$ ) to be favorable to Democrats and the other half to be favorable to Republicans. Participants were randomly assigned to either judge each headline's veracity (Accuracy condition) or indicate if they would consider sharing each headline online (Sharing condition); for details, see Methods. Unless otherwise noted, all $p$ values are generated by linear regression with robust standard errors clustered on participant and 65 headline.

In the Accuracy condition (Fig 1a), true headlines were rated as accurate significantly more often than false headlines (55.9 percentage point difference, $F(1,36172)=375.05$, $\mathrm{p}<0.0001$ ). Although politically concordant headlines were also rated as accurate significantly more often than politically discordant headlines (10.1 percentage point difference, $F(1,36172)=26.45, \mathrm{p}<0.0001$ ), this difference based on partisan alignment was significantly smaller than the 55.9 percentage point difference between true and false headlines $(F(1,36172)=137.26, \mathrm{p}<0.0001)$. Turning to the 
Sharing condition (Fig 1b), we see the opposite pattern: Whether the headline was politically concordant or discordant had a significantly larger effect on sharing intentions (19.3 percentage points) than whether the headline was true or false (5.9 percentage points; $F(1,36172)=19.73$, $\mathrm{p}<0.0001)$. Accordingly, the effect of headline veracity was significantly larger in the accuracy condition than the sharing condition, $F(1,36172)=260.68, p<.0001$, while the effect of concordance was significantly larger in the sharing condition than the accuracy condition, $F(1,36172)=17.24, p<.0001$; for full regression table and robustness checks, see SI Section 2. Notably, the pattern of sharing intentions we observe here matches the pattern of actual sharing observed in a large-scale analysis of Twitter users, where partisanship was found to be a much stronger predictor of sharing than veracity ${ }^{20}$.

To illustrate the disconnect between accuracy judgments and sharing intentions, consider, for example, the following headline: "Over 500 'Migrant Caravaners' Arrested With Suicide Vests". This was rated as accurate by $15.7 \%$ of Republicans in our study, but $51.1 \%$ of Republicans said they would consider sharing it. Thus, the results from Study 1 suggest that the confusion-based account cannot fully explain the sharing of misinformation: our participants were more than twice as likely to consider sharing false but politically concordant headlines $(37.4 \%)$ as they were to rate such headlines as accurate $(18.2 \%) ; F(1,36172)=19.73, \mathrm{p}<0.0001$.

One possible explanation for this dissociation between accuracy judgments and sharing intentions is offered by the preference-based account of misinformation sharing. By this account, people care about accuracy much less than other factors (e.g., partisanship), and therefore knowingly share misinformation. The fact that participants in Study 1 were willing to share ideologically consistent but false headlines could thus be reasonably construed as revealing their preference for weighing non-accuracy dimensions (such as ideology) over accuracy. Yet when asked at the end of the study whether it is important to only share content that is accurate on social media, the modal response was "extremely important" (see Extended Data Figure 1). A similar pattern was observed in a more nationally representative sample of $N=401$ Americans from Lucid ${ }^{21}$ in Study 2, who rated accuracy as substantially more important for social media sharing than any of the other dimensions that we asked about (paired $t$-tests, $p<.001$ for all comparisons; Fig 1c); for design details, see Methods.

Why, then, were participants in Study 1 - and millions of other Americans in recent years - so willing to share misinformation? In answer, we advance the inattention-based account, whereby (i) people do care more about accuracy than other content dimensions, but accuracy nonetheless often has little impact on sharing because (ii) the social media context focuses their attention on other factors such as the desire to attract and please followers/friends ${ }^{22}$, or to signal one's group membership ${ }^{23}$. In the language of utility theory, we argue that an "attentional spotlight" is shone upon certain terms in the decider's utility function, such that only those terms are weighed when making a decision (for a mathematical model, see SI Section 3). 


\section{Priming accuracy improves sharing}

We differentiate between these theories by subtly inducing people to think about accuracy, which the preference-based account predicts should have no effect whereas the inattention-based account predicts should increase the accuracy of content that is shared (see SI Section 3.2). We first test these competing predictions in a series of survey experiments. In the Control condition of each experiment, participants were shown 24 news headlines (balanced on veracity and partisanship, as in Study 1) and asked how likely they would be to share each headline on Facebook. In the Treatment, participants were asked to rate the accuracy of a single non-partisan news headline at the outset of the study (ostensibly as part of a pretest for stimuli for another study). They then went on to complete the same sharing intentions task as in the Control condition - but with the concept of accuracy more likely to be salient in their minds. For details of the experimental design, see Methods.

In two experiments using Americans recruited from MTurk (Study 3, $N=727$; Study 4, $N=780$ ), we find that the Treatment significantly increased sharing discernment (Fig 2a,b; interaction between headline veracity and treatment: $\mathrm{S} 3, b=0.053[0.032,0.074], F(1,17413)=24.21, p<.0001$; S4, $b=0.065[0.036,0.094], F(1,18673)=19.53, p<.0001)$. Specifically, participants in the Treatment were significantly less likely to consider sharing false headlines compared to the Control (S3, $b=-.055[-.083,-.026], F(1,17413)=14.08, p=.0002$; S4, $b=-0.058[-0.091,-0.025]$, $F(1,18673)=11.99, p=.0005)$, but equally likely to consider sharing true headlines $(\mathrm{S} 3, \mathrm{~b}=-0.002$ $[-.031, .028], F(1,17413)=.01, p=.92 ; \mathrm{S} 4, b=0.007$ [-0.020, 0.033], $F(1,18673)=.23, p=.63)$. As a result, sharing discernment (the difference in sharing intentions for true versus false headlines) was 2.0 times larger in the Treatment relative to the Control in Study 3, and 2.4 times larger in Study 4. Furthermore, the treatment effect was significantly larger for politically concordant headlines compared to politically discordant headlines $(b=0.022$ [0.012, 0.033], $F(1,36078)=$ $18.09, \mathrm{p}<0.0001)$, and significantly increased discernment for both Democrats $(b=0.069[0.048$, $0.091], F(1,24636)=40.38, p<.0001)$ and Republicans $(b=0.035$ [0.007, 0.063], $F(1,11394)=5.93$, $p=0.015$ ). See SI Section 2 for full regression table.

Importantly, there was no significant difference between conditions in responses to a postexperimental question regarding the importance of only sharing accurate content ( $t$-test: $t(1498)=.42, p=.68,95 \% \mathrm{CI}[-0.075,0.115]$ points on a $1-5$ scale; Bayesian independent samples $t$ test with Cauchy prior distribution with interquartile range of 0.707 : $\mathrm{BF}_{10}=0.063$, providing strong evidence for the null), or regarding participants' perceptions of the importance their friends place on only sharing accurate content $(t$-test: $t(768)=-.57, p=.57,95 \%$ CI $[-0.205,0.113]$ points on a 1 5 scale; Bayesian independent samples $t$-test with Cauchy prior distribution with interquartile range of $0.707: \mathrm{BF}_{10}=0.095$, providing strong evidence for the null).

Our next survey experiment (Study $5, N=1,268$ ) tested whether the previous results generalize to a more representative sample by recruiting participants from Lucid $^{21}$ that were quota-sampled to 
match the distribution of American residents on age, gender, ethnicity, and geographic region. Study 5 also included an Active Control condition in which participants were asked to rate the humorousness (rather than accuracy) of a single non-partisan news headline at the outset of the study, and an Importance Treatment that tested another approach for making accuracy salient by having participants begin the study by indicating the importance they place on only sharing accurate content (instead of rating the accuracy of a neutral headline). The results (Figure 2c) successfully replicated Studies 3 and 4 . As expected, there were no significant differences in sharing intentions between the Control and the Active Control conditions (interaction between veracity and condition, $b=.015[-.043, .059], F(1,6772)=0.04, p=.84)$; and both treatments significantly increased sharing discernment relative to the controls (interaction between veracity and condition: Treatment, $b=0.054$ [0.023, 0.085], $F=11.98, p=.0005$; Importance Treatment, $b=0.038[0.014,0.061], F=9.76, p=.0018)$. See SI Section 2 for full regression table.

\section{Attending to accuracy as the mechanism}

Next, we provide evidence that shifting attention to accuracy is the mechanism behind this effect by showing that the Treatment leads to the largest reduction in the sharing of headlines that participants are likely to deem to be the most inaccurate (and vice versa for the most plainly accurate headlines). A headline-level analysis finds a positive correlation between the Treatment's effect on sharing and the headline's perceived accuracy (as measured in pre-tests, see SI Section 1 for details): Study 3, $r(22)=.71, p=.0001$; Study 4, $r(22)=.67, p=.0003$; Study $5, r(18)=.61, p=.005$; see Figure 3a-c. That is, the most obviously inaccurate headlines are the ones that the accuracy salience treatment most effectively discourages people from sharing.

Furthermore, fitting our formal limited-attention utility model to the experimental data provides quantitative evidence against the preference-based account (participants value accuracy as much as or more than partisanship) and for the inattention-based account (participants often fail to consider accuracy); see Extended Data Table 1 and SI Sections 3.5 and 3.6.

In Study 6, we present a final survey experiment ( $N=710$ Americans from MTurk) that quantifies the relative contribution of the confusion-based, preference-based, and inattention-based accounts to the willingness to share false headlines on social media. To do so, we compare the Control condition to a Full Attention Treatment, in which participants are asked to assess the accuracy of each headline immediately before deciding whether they would share it; for details, see Methods. As illustrated in Figure 3d, the results show that, of the sharing intentions for false headlines, the inattention-based account explains $51.2 \%(95 \%$ CI [38.4\%, 62.0\%]) of sharing, the confusionbased account explains $33.1 \%$ (95\% CI [25.1\%, 42.4\%]) of sharing, and the preference-based account explains only $15.8 \%$ (95\% CI [11.1\%, 21.5\%]) of sharing. Thus, inattention does not merely operate on the margin, but rather plays a central role in the sharing of misinformation in our experimental paradigm. Furthermore, the preference-based account's low level of explanatory power relative to the inattention-based account in Study 6 is consistent with the model fitting 
results in Extended Data Table 1 and SI Section 3.6 described above - thus providing convergent evidence against the preference-based account being a central driver of misinformation sharing.

\section{Deploying the intervention on Twitter}

Finally, to test whether our findings generalize to natural social media use settings (rather than laboratory experiments), actual (rather than hypothetical) sharing decisions, and misinformation more broadly (rather than just blatant "fake news"), in Study 7 we conducted a digital field experiment on social media ${ }^{24}$. To do so, we selected $N=5,379$ Twitter users who had previously shared links to two particularly well-known right-leaning sites that professional fact-checkers have rated as highly untrustworthy ${ }^{25}$ : Breitbart.com and Infowars.com. We then sent these users private messages asking them to rate the accuracy of a single non-political headline (Fig. 4a), and used a stepped-wedge (i.e., randomized roll-out) design to observe the message's causal impact on the quality of the news content the users subsequently shared (based on domain-level ratings of professional fact-checkers ${ }^{25}$ ); for details of the experimental design, see Methods.

Examining baseline (pre-treatment) sharing behavior shows that we were successful in identifying users with relatively low-quality news-sharing habits: The average quality score of news sources from pre-treatment posts was 0.34 . (For comparison, the fact-checker-based quality score was 0.02 for Infowars; 0.16 for Breitbart; 0.39 for Fox News, and 0.93 for the New York Times.) Moreover, $46.6 \%$ of shared news sites were sites that publish false or misleading content $(0.9 \%$ fake news sites, $45.7 \%$ hyperpartisan sites).

Consistent with our survey experiments, we find clear evidence that the single accuracy message made users more discerning in their subsequent sharing decisions (exact $p$-values, $p_{F R I}$, determined using Fisherian Randomization Inference ${ }^{26}$ ). Relative to baseline, the accuracy message increased the average quality of the news sources shared $\left(b=0.007, t(5375)=2.91, C I_{\text {Null }}=[-0.44,2.59]\right.$, $\left.p_{F R}=.009\right)$ and the total quality of shared sources summed over all posts $(b=0.014, t(5375)=3.12$, $\left.C I_{\text {Null }}=[-0.08,2.90], p_{F R F}=.011\right)$. This translates into increases of $4.8 \%$ and $9.0 \%$ respectively when estimating the treatment effect for user-days on which tweets would occur in treatment (that is, excluding user-days in the "never-taker" principal stratum ${ }^{27,28}$, because the treatment cannot have an effect when no tweets would occur in either treatment or control); including user-days with no tweets yields an increase of $2.1 \%$ and $4.0 \%$ in average and total quality, respectively. Furthermore, the level of sharing discernment (i.e., difference in number of mainstream versus fake/hyperpartisan links shared per user-day; interaction between post-treatment dummy and link type) was 2.8 times higher after receiving the accuracy message $\left(b=0.059, t(5371)=3.27, C I_{\text {Null }}=[-\right.$ $\left.0.31,2.67], p_{F R F}=.003\right)$.

To provide further support for the inattention-based account, we contrast low-engagement sharing (where the user simply re-shares content posted by another user: i.e., retweets without comment) with high-engagement sharing (where the poster invests some time and effort to craft their own 
post or add a comment to another post). Low-engagement sharing, which accounts for $72.4 \%$ of our dataset, presumably involves less attention than high-engagement sharing - therefore the inattention-based account of misinformation sharing predicts that our manipulation should primarily affect low-engagement sharing. Consistent with this prediction, we observe a significant positive interaction $\left(b=0.008, t(5371)=2.78, C I_{\text {Null }}=[-0.80,2.24], p_{F R F}=.004\right)$, such that the treatment increases average quality of low-engagement sharing but not high-engagement sharing. Furthermore, we found no significant treatment effect on the number of posts without links to any of the 60 rated news sites $\left(b=0.266, t(5375)=0.50, C I_{\text {Null }}=[-1.11,1.64], p_{F R I}=.505\right)$.

Importantly, the significant effects we observed are not unique to one particular set of analytic choices. Figure $4 \mathrm{~b}$ shows the distribution of $p$-values observed in 192 different analyses assessing the overall treatment effect on average quality, summed quality, or discernment under a variety of analytic choices. Of these analyses, $82.3 \%$ indicate a significant positive treatment effect (and none of 32 analyses of posts without links to a rated site - in which we would not expect a treatment effect - find a significant difference). For details, see Extended Data Table 4 and SI Section 5.

Finally, we examine the data at the level of the domain (Fig. 4c). We see that the treatment effect is driven by increasing the fraction of rated-site posts with links to mainstream new sites with strong editorial standards such as the New York Times, and decreasing the fraction of rated-site posts that linked to relatively untrustworthy hyperpartisan sites such as Breitbart. Indeed, a domain-level pairwise correlation between fact-checker rating and change in sharing due to the intervention shows a very strong positive relationship (domains weighted by number of pretreatment posts; $r(44)=0.74, p<.0001)$, replicating the increase in sharing discernment observed in the survey experiments (Figure 3A-C). In sum, our accuracy message successfully induced Twitter users who regularly shared misinformation to increase the quality of the news they shared.

In SI Section 6, we use computational modeling to connect our empirical observations about individual-level sharing decisions in Study 7 to the network-level dynamics of misinformation spread. Across a variety of network structures, we observe that network dynamics can substantially amplify the magnitude of treatment effects on sharing (see Extended Data Figure 6). Improving the quality of the content shared by one user improves the content that their followers see, and therefore improves the content their followers share. This in turn improves what the followers' followers see and share, and so on. Thus, the cumulative effects of such an intervention on how misinformation spreads across networks may be substantially larger than what is observed when only examining the treated individuals - particularly given that, in Study 7, we find that the treatment is as effective, if not more so, for users with larger numbers of followers (see SI Section $5)$.

\section{Conclusion}


273 Together, these studies suggest that people are often distracted from considering the content's 274 accuracy by other factors when deciding what to share on social media. Therefore, shifting 275 attention to the concept of accuracy can cause people to improve the quality of the news they share. 276 Furthermore, we found a dissociation between accuracy judgments and sharing intentions which 277 suggests that people may share news that they do not necessarily have a firm belief in. As a 278 consequence, people's beliefs may not be as partisan as their social media feeds seem to indicate. 279 Future work is needed to more precisely identify people's state of belief when not reflecting on 280 accuracy: Is it that people hold no particular belief one way or the other, or that they tend to assume 281 content is true by default ${ }^{29}$ ?

283 A substantial limitation of our studies is that they are focused on political news sharing among 284 Americans. In a recent set of follow-up survey experiments, our findings of a disconnect between accuracy and sharing judgments in Study 1 and our treatment increasing sharing discernment in Studies 3, 4 and 5 were successfully replicated using headlines about COVID-19 with an American sample $^{7}$. Future work should examine applications to other content domains, including misinformation from political elites (e.g., about fraud in the 2020 U.S. Presidential Election ${ }^{30}$, and explore cross-cultural generalizability. Extending the Twitter field experiment design used in Study 7 is also a promising direction for future work, including using a more continuous shockbased model of how (and when) the treatment affects individual rather than the conservative intentto-treat approach used here, generalizing beyond users who follow-back experimenter accounts, testing an active control, and using article-level quality rather than domain-level quality scores.

Our results suggest that the current design of social media platforms - in which users scroll quickly through a mix of serious news and emotionally engaging content, and receive instantaneous quantified social feedback on their sharing - may discourage people from reflecting on accuracy. But this need not be the case. Our treatment translates easily into interventions that social media platforms could employ to increase users' focus on accuracy. For example, platforms could periodically ask users to rate the accuracy of randomly selected headlines, thus reminding them about accuracy in a subtle way that should avoid reactance ${ }^{31}$ (and simultaneously generating useful crowd ratings that can help identify misinformation ${ }^{25,32}$ ). Such an approach could potentially increase the quality of news circulating online without relying on a centralized institution to certify

304 truth and censor falsehood. 
1. Lazer, D. et al. The science of fake news. Science (80-. ). 9, 1094-1096 (2018).

2. Lederer, E. UN chief says misinformation about COVID-19 is new enemy. ABC News (2020). Available at: https://abcnews.go.com/US/wireStory/chief-misinformation-covid-19-enemy69850124. (Accessed: 4th April 2020)

3. Pasquetto, I. et al. Tackling misinformation: What researchers could do with social media data. Harvard Kennedy Sch. Misinformation Rev. 1, (2020).

4. Pennycook, G. \& Rand, D. G. The Cognitive Science of Fake News. PsyArXiv 1-29 (2020). doi:10.31234/OSF.IO/AR96C

5. Guess, A. M. et al. A digital media literacy intervention increases discernment between mainstream and false news in the United States and India. Proc. Natl. Acad. Sci. 201920498 (2020). doi:10.1073/pnas.1920498117

6. Kozyreva, A., Lewandowsky, S. \& Hertwig, R. Citizens versus the internet: confronting digital challenges with cognitive tools. Psychol. Sci. Public Interest 21, 103-156 (2020).

7. Pennycook, G., McPhetres, J., Zhang, Y., Lu, J. G. \& Rand, D. G. Fighting COVID-19 misinformation on social media: Experimental evidence for a scalable accuracy nudge intervention. Psychol. Sci. (2020). doi:10.31234/OSF.IO/UHBK9

8. Van Bavel, J. J. \& Pereira, A. The partisan brain: An Identity-based model of political belief. Trends Cogn. Sci. (2018).

9. Kahan, D. M. Misconceptions, Misinformation, and the Logic of Identity-Protective Cognition. SSRN Electron. J. (2017). doi:10.2139/ssrn.2973067

10. Pennycook, G., Cannon, T. D. \& Rand, D. G. Prior Exposure Increases Perceived Accuracy of Fake News. J. Exp. Psychol. Gen. (2018). doi:10.1037/xge0000465

11. McGrew, S., Ortega, T., Breakstone, J. \& Wineburg, S. he Challenge That's Bigger than Fake News: Civic Reasoning in a Social Media Environment. Am. Educ. 41, 4-9 (2017).

12. Lee, N. M. Fake news, phishing, and fraud: a call for research on digital media literacy education beyond the classroom. Commun. Educ. 67, 460-466 (2018).

13. McDougall, J., Brites, M. J., Couto, M. J. \& Lucas, C. Digital literacy, fake news and education. Cult. Educ. 31, 203-212 (2019).

14. Jones-Jang, S. M., Mortensen, T. \& Liu, J. Does Media Literacy Help Identification of Fake News? Information Literacy Helps, but Other Literacies Don't. Am. Behav. Sci. 000276421986940 (2019). doi: $10.1177 / 0002764219869406$

15. Redlawsk, D. Hot cognition or cool consideration? Testing the effects of motivated reasoning on political decision making. J. Polit. 64, 1021-1044 (2002).

16. Strickland, A. A., Taber, C. S. \& Lodge, M. Motivated Reasoning and Public Opinion. J. Health Polit. Policy Law 36, 89-122 (2011).

17. Horton, J., Rand, D. \& Zeckhauser, R. The online laboratory: Conducting experiments in a real labor market. Exp. Econ. 14, 399-425 (2011).

18. Pennycook, G. \& Rand, D. G. Lazy, not biased: Susceptibility to partisan fake news is better explained by lack of reasoning than by motivated reasoning. Cognition 188, 39-50 (2019).

19. Pennycook, G., Bear, A., Collins, E. \& Rand, D. G. The Implied Truth Effect: Attaching Warnings to a Subset of Fake News Stories Increases Perceived Accuracy of Stories Without Warnings. Manage. Sci. (2020). doi:10.1287/mnsc.2019.3478

20. Grinberg, N., Joseph, K., Friedland, L., Swire-Thompson, B. \& Lazer, D. Fake news on twitter during the 2016 U.S. Presidential election. Science (80-. ). 363, 374-378 (2019).

21. Coppock, A. \& Mcclellan, O. A. Validating the Demographic, Political, Psychological, and Experimental Results Obtained from a New Source of Online Survey Respondents. Res. Polit. (2019).

22. Marwick, A. E. \& Boyd, D. I tweet honestly, I tweet passionately: Twitter users, context collapse, 
and the imagined audience. New Media Soc. 13, 114-133 (2011).

23. Donath, J. \& Boyd, D. Public displays of connection. BT Technol. J. 22, 71-82 (2004).

24. Munger, K. Tweetment Effects on the Tweeted: Experimentally Reducing Racist Harassment. Polit. Behav. 39, 629-649 (2017).

25. Pennycook, G. \& Rand, D. G. Fighting misinformation on social media using crowdsourced judgments of news source quality. Proc. Natl. Acad. Sci. (2019). doi:10.1073/pnas.1806781116

26. Fisher, R. A. The design of experiments. (Oliver and Boyd, 1937).

27. Angrist, J. D., Imbens, G. W. \& Rubin, D. B. Identification of Causal Effects Using Instrumental Variables. J. Am. Stat. Assoc. 91, 444 (1996).

28. Frangakis, C. E. \& Rubin, D. B. Principal stratification in causal inference. Biometrics 58, 21-9 (2002).

29. Gilbert, D. T. How mental systems believe. Am. Psychol. 46, 107-119 (1991).

30. Pennycook, G. \& Rand, D. G. Examining false beliefs about voter fraud in the wake of the 2020 Presidential Election. Harvard Kennedy Sch. Misinformation Rev. 1-22 (2021). doi:10.37016/mr2020-51

31. Mosleh, M., Martel, C., Eckles, D. \& Rand, D. G. Perverse Consequences of Debunking in a Twitter Field Experiment: Being Corrected for Posting False News Increases Subsequent Sharing of Low Quality, Partisan, and Toxic Content. Proc. 2021 CHI Conf. Hum. Factors Comput. Syst.

32. Allen, J., Arechar, A. A., Pennycook, G. \& Rand, D. G. Scaling up fact-checking using the wisdom of crowds. PsyArXiv Work. Pap. (2020). 

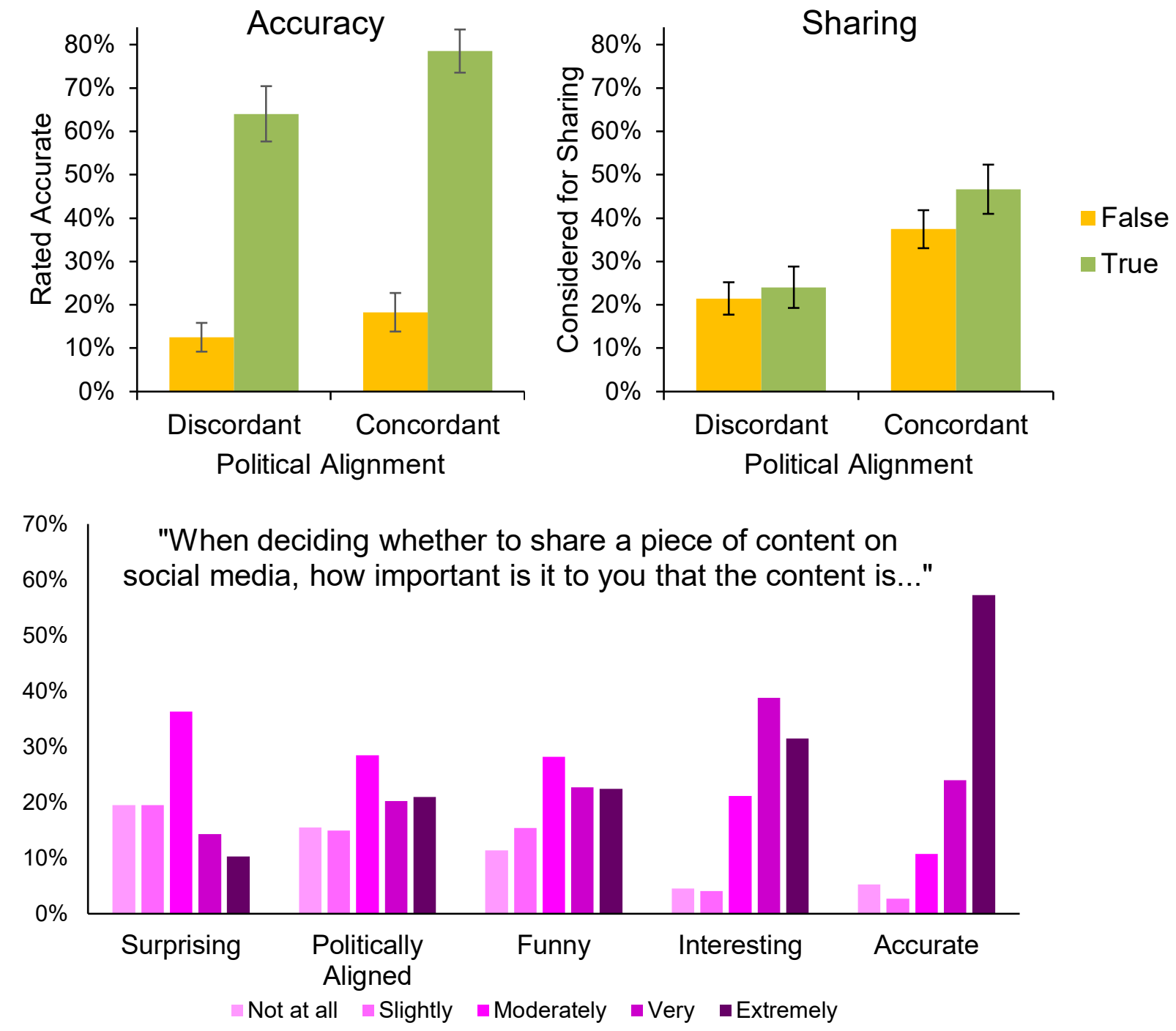

Figure 1. Participants can easily identify false headlines when asked to judge accuracy; however, veracity has little impact on sharing intentions, despite an overall desire to only share accurate content. In Study 1, $\mathrm{N}=1,002$ Americans from Amazon Mechanical Turk were presented with a set of 36 headlines and either asked to indicate if they thought the headlines were accurate or if they would consider sharing them on social media. (A) Shown is the fraction of headlines rated as accurate in the Accuracy condition, by the veracity of the headline and political alignment between the headline and the participant. Participants were significantly more likely to rate true headlines as accurate compared to false headlines (55.9 percentage point difference, $F(1,36172)=375.05, p<0.0001$ ), whereas the partisan alignment of the headlines had a significantly smaller impact (10.1 percentage point difference, $F(1,36172)=26.45, p<0.0001$; interaction, $F(1,36172)=137.26, p<0.0001)$. (B) Shown is the fraction of headlines participants said they would consider sharing in the Sharing condition, by the veracity of the headline and political alignment between the headline and the participant. In contrast to the Accuracy condition, the effect of headline veracity was significantly smaller in the sharing condition, $F(1,36172)=260.68, \mathrm{p}<.0001$, whereas the effect of political concordance was significantly larger, $F(1,36172)=17.24, \mathrm{p}<.0001$. Error bars indicate $95 \%$ confidence intervals based on standard errors clustered on participant and headline. (C) Participants nonetheless overwhelmingly said they thought that accuracy was more important on average than partisanship (and all other content dimensions we asked about) when making social media sharing decisions. 

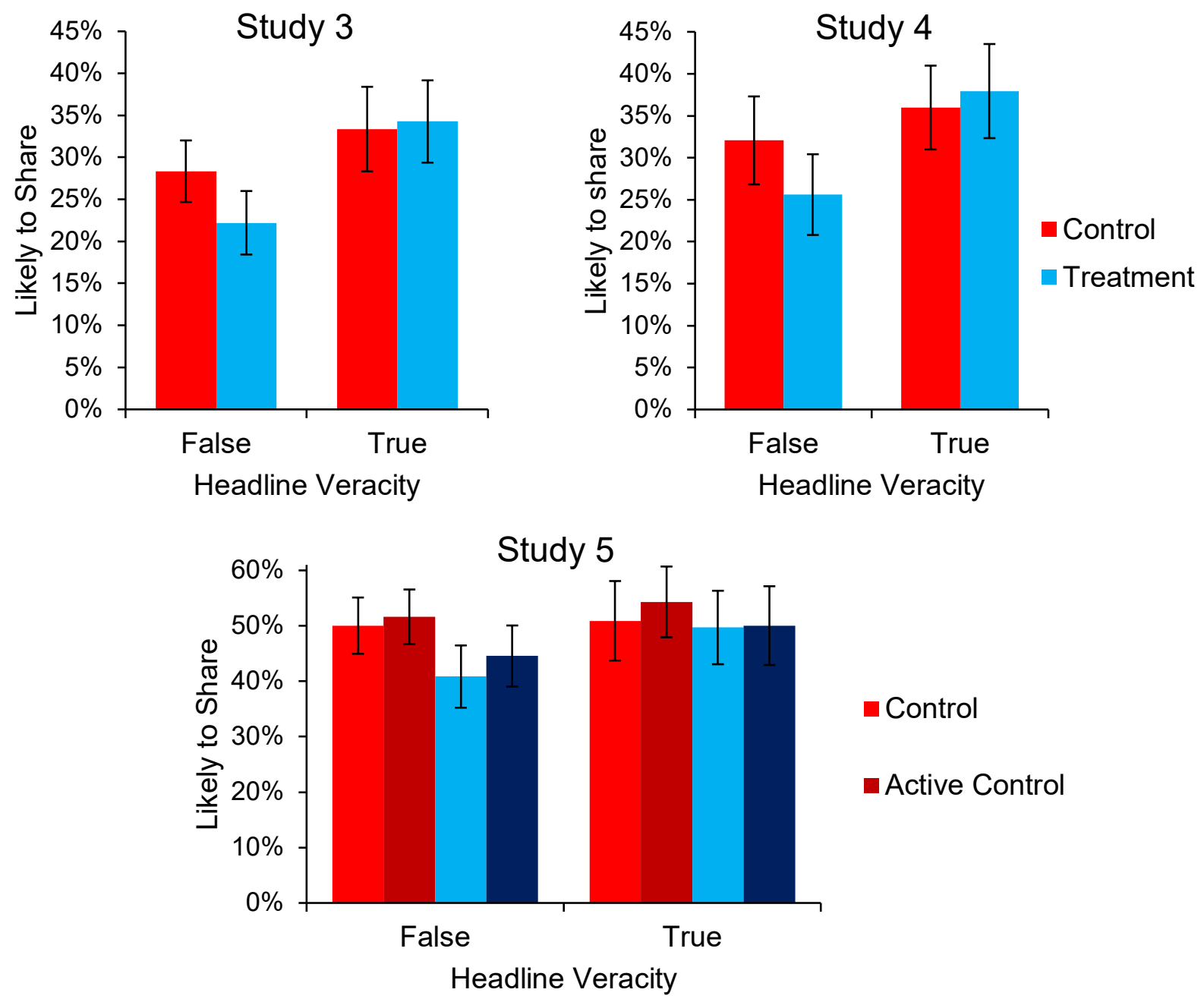

Figure 2. Inducing survey respondents to think about accuracy increases the veracity of headlines they are willing to share. Participants in Studies 3 (A; N=727 Americans from MTurk), Study 4 (B; N=780 Americans from MTurk), and Study 5 (C; N=1,268 Americans from Lucid, nationally representative on age, gender, ethnicity, and geographic region) indicated how likely they would be to consider sharing a series of actual headlines from social media. Participants in the Treatment rated the accuracy of a single non-political headline at the outset of the study, thus increasing the likelihood that they would think about accuracy when indicating sharing intentions relative to the Control. In Study 5, we added an Active Control (in which participants rated the humorousness of a single headline at the outset of the study) and an Importance Treatment (in which participants were asked at the study outset how important they thought it was to only share accurate content). For interpretability, shown here is the fraction of "likely" responses (responses above the midpoint of the 6-point Likert scale) by condition and headline veracity; the full distribution of responses are shown in Extended Data Figures 2 and 3. As per our preregistered analysis plans, these analyses focus only on participants who indicated that they sometimes consider sharing political content on social media; for analysis including all participants, see SI Section 2. Error bars indicate $95 \%$ confidence intervals 

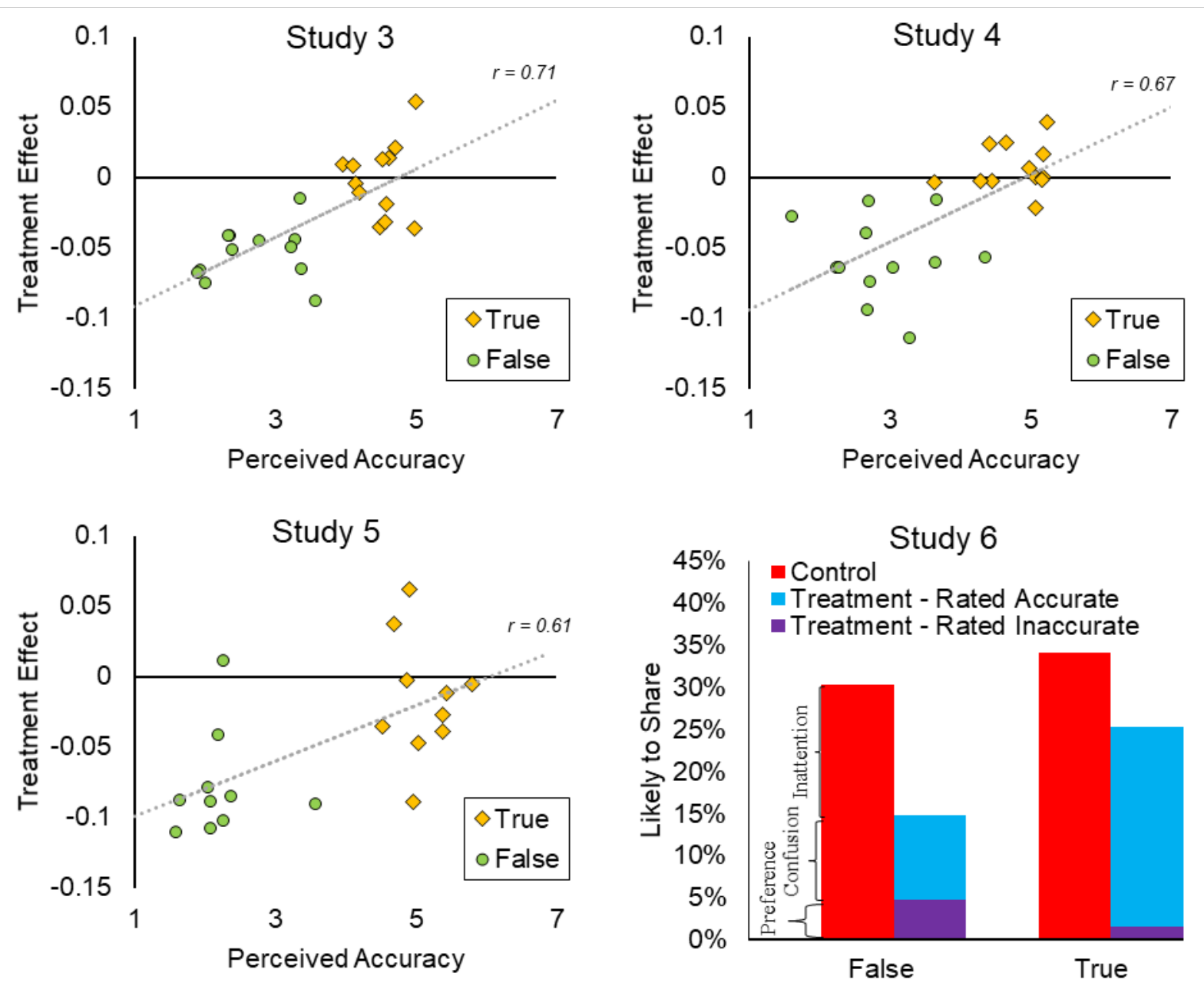

Figure 3. Inattention plays an important role in the sharing of misinformation. There is a significant positive correlation across headlines between the average out-of-sample accuracy rating and the effect of the treatment in Study 3 (A, $r(22)=.71, p=.0001)$, Study 4 (B, $r(22)=.67, p=.0003$ ), and Study $5(\mathrm{C}, r(18)=.61, p=.005)$ : The accuracy reminder caused a larger decrease in sharing intentions for items that were deemed to be more unlikely. This observation supports our argument that the Treatment intervention operated via focusing attention on accuracy, and that many people do not want to share content they think is inaccurate. As shown in Extended Data Figure 4, in Study 5 a similar pattern was found for the Important Treatment, and no such effect existed for the Active Control. (D) In Study 6, participants rated the accuracy of each headline (a Full Attention Treatment) before making a judgment about sharing. This allows us to distinguish between false items that: a) participants share despite believing to be inaccurate (i.e., a preference-based rejection of truth), b) participants share and also believe to be accurate (i.e., confusion-based), and c) participants no longer shared once they considered accuracy (i.e., inattentionbased). Results indicate that, among the false headlines that are shared in the Control, most are shared due to inattention $(51.2 \%)$, fewer are shared because of confusion (33.1\%), and a small minority are shared because of a preference to share false content (15.8\%). Bootstrapping simulations (10,000 repetitions) find that inattention explains marginally significantly more misinformation sharing than confusion $(b=.181[-0.036,0.365], p=0.098)$ and significantly more than purposeful sharing $(b=.354[0.178,0.502], p=0.0004)$; and that confusion explains 

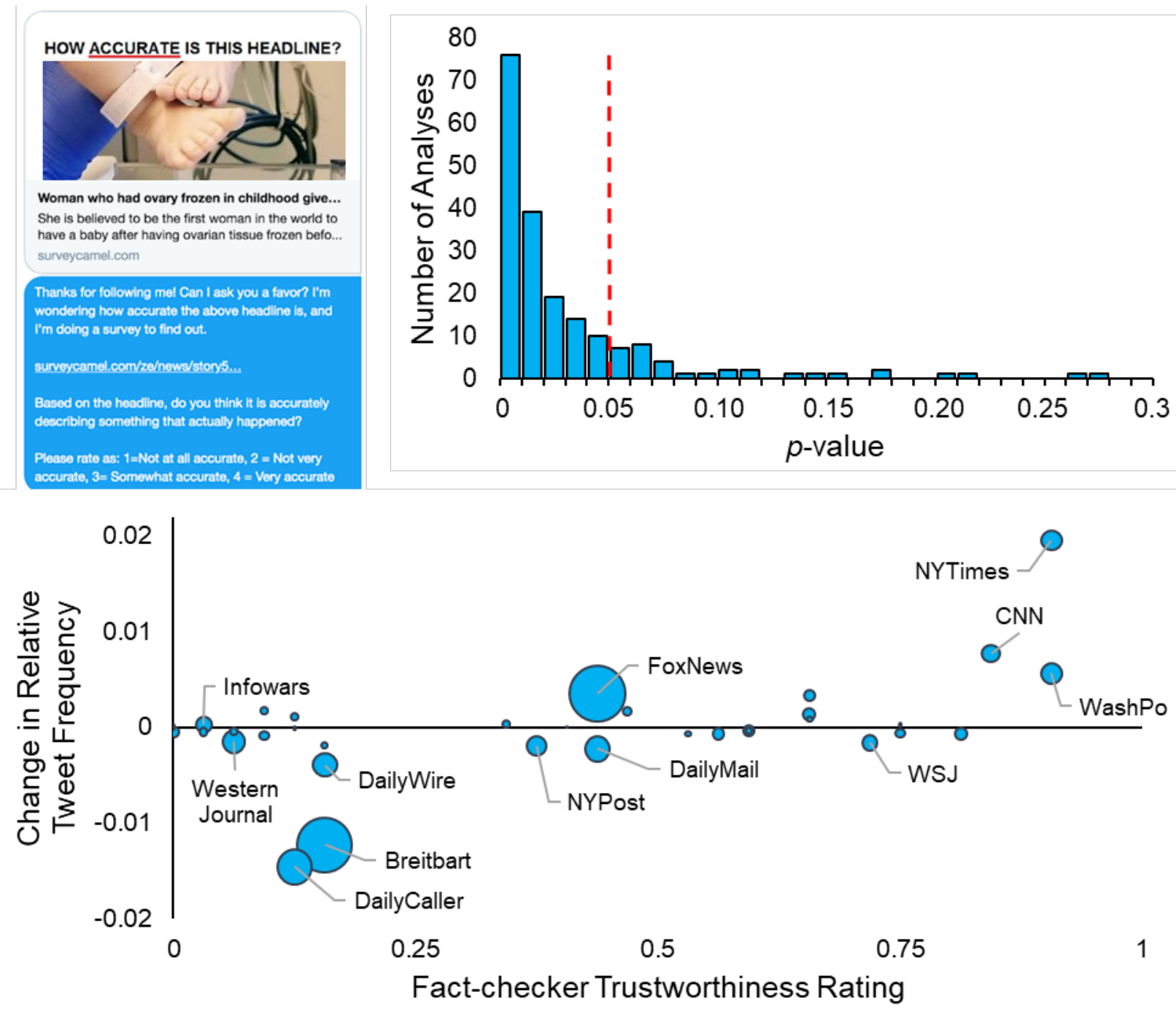

Figure 4. Sending Twitter users a message asking for their opinion about the accuracy of a single nonpolitical headline increases the quality of the news they subsequently share. In Study 7, we conducted an experiment on the Twitter platform involving $\mathrm{N}=5,379$ users who had recently shared links to websites that regularly produce misleading and hyperpartisan content. We randomized the date on which users were sent an unsolicited message asking them to rate the accuracy of a single non-political headline. We then compared the quality of the news sites shared in the 24 hours after receiving the message to the sites shared by participants who had not yet received the message. (A) The private message sent to the users is shown here. We did not expect most users to respond to the message, or even read it in its entirety. Thus we designed it such that reading only the top line should be sufficient to shift attention to the concept of accuracy. (B) To test the robustness of our results, we conducted 192 analyses that differed in their dependent variable, inclusion criteria and model specifications. Shown here is the distribution of $p$-values resulting from each of these analyses. Over $80 \%$ of approaches yield $p<0.05$. (C) A domain-level analysis provides a more detailed picture of the effect of the intervention. The $\mathrm{x}$-axis indicates the trust score given to each outlet by professional fact-checkers. The y-axis indicates the fraction of rated links to each 
Preregistrations for all studies are available at https://osf.io/p6u8k/. In all survey experiments, we do not exclude participants for inattentiveness or straightlining to avoid selection effects that can undermine causal inference. The researchers were not blind to the hypotheses when carrying out

\section{Study 1}

In Study 1, participants were presented with a pretested set of false and true headlines (in "Facebook format") and were either asked to indicate whether they thought they were accurate or not, or whether they would consider sharing them on social media or not. Our prediction was that the difference in 'yes' responses between false and true news (i.e., discernment) will be greater when individuals are asked about accuracy than when they are asked about sharing, whereas the difference between ideological discordant and concordant news (i.e., bias) will be greater when they are asked about sharing than when they are asked about accuracy.

\section{Participants}

We preregistered a target sample of 1,000 complete responses, using participants recruited from Amazon's Mechanical Turk (MTurk) but noted that we would retain individuals who completed the study above the 1,000-participant quota. In total, 1,825 participants began the survey. However, an initial (pre-treatment) screener only allowed American participants who indicated having a Facebook or Twitter account (when shown a list of different social media platforms) and indicated that they would consider sharing political content (when shown a list of different content types) to continue and complete the survey. The purpose of these screening criteria was to focus our investigation on the relevant subpopulation - those who share political news. The accuracy judgments of people who never share political news on social media are not relevant here, given our interest in the sharing of political misinformation. Of the participants who entered the survey, 153 indicated that they had neither a Facebook nor Twitter account, and 651 indicated that they did have either a Facebook or Twitter account but would not consider sharing political content. A further 16 participants passed the screener but did not finish the survey and thus were removed from the data set. The full sample (Mean age $=36.7$ ) included 475 males, 516 females, and 14 participants who selected another gender option. This study was run on August $13^{\text {th }}, 2019$.

\section{Materials}

We presented participants with 18 false ("fake") and 18 true ("real") news headlines in a random order for each participant. The false news headlines were originally selected from a third-party fact-checking website, Snopes.com, and were therefore verified as being fabricated and untrue. The true news headlines were all accurate and selected from mainstream news outlets to be roughly contemporary with the false news headlines. Moreover, the headlines were selected to be either Pro-Democratic or Pro-Republican (and equally so). This was done using a pretest, which confirmed that the headlines were equally partisan across the categories (for a similar approach, see ${ }^{10,18,19}$. See SI Section 1 for details about the pretest. 
Participants in Study 1 were also asked: "How important is it to you that you only share news articles on social media (such as Facebook and Twitter) if they are accurate", to which they responded on a 5-point scale from 'not at all important' to 'extremely important'. We also asked participants about their frequency of social media use, along with several exploratory questions about media trust. At the end of the survey, participants were asked if they responded randomly at any point during the survey or searched for any of the headlines online (e.g., via Google). As noted in our preregistration, we did not intend to exclude these individuals. Participants also completed several additional measures as part of separate investigations (this was also noted in the preregistration); namely, the 7 -item Cognitive Reflection Test ${ }^{18}$, a political knowledge questionnaire, and the positive and negative affective schedule ${ }^{33}$. In addition, participants were asked several demographic questions (age, gender, education, income, and a variety of political and religious questions). The most central political partisanship question was "Which of the following best describes your political preference" followed by the following response options: Strongly Democratic, Democratic, Lean Democratic, Lean Republican, Republican, Strongly Republican. For purposes of data analysis, this was converted to a Democratic/Republican binary. The survey was completed on August $13^{\text {th }}-14^{\text {th }}, 2019$. The full survey is available online in both text format and as a Qualtrics file, along with all data (https://osf.io/p6u $8 \mathrm{k} /$ ).

\section{Procedure}

Participants in the accuracy condition were given the following instructions: "You will be presented with a series of news headlines from 2017 to 2019 (36 in total). We are interested in whether you think these headlines describe an event that actually happened in an accurate and unbiased way. Note: The images may take a moment to load." In the sharing condition, the middle sentence was replaced with "We are interested in whether you would consider sharing these stories on social media (such as Facebook or Twitter)." We then presented participants with the full set of headlines in a random order. In the accuracy condition, participants were asked "To the best of your knowledge, is this claim in the above headline accurate?" In the sharing condition, participants were asked "Would you consider sharing this story online (for example, through Facebook or Twitter)?" Although these sharing decisions are hypothetical, headline-level analyses suggest that self-report sharing decisions of news articles like those used in our study correlate strongly with actual sharing on social media ${ }^{34}$.

In both conditions, the response options were simply "No" and "Yes." Moreover, participants either saw the response options listed as Yes/No or No/Yes (randomized across participants i.e., an individual participant only ever saw 'yes' first or 'no' first).

This study was approved by the University of Regina Research Ethics Board (Protocol \#2018116).

\section{Analysis plan}

Our preregistration specified that all analyses would be performed at the level of the individual item (i.e., one data point per item per participant; $0=$ No, $1=$ Yes) using linear regression with robust standard errors clustered on participant. However, we subsequently realized that we 
should also be clustering standard errors on headline (as multiple ratings of the same headline are non-independent in a similar way to multiple ratings from the same participant), and thus deviated from the preregistrations in this minor way (all key results are qualitatively equivalent if only clustering standard errors on participant). The linear regression was preregistered to have the following independent variables: a condition dummy $(-0.5=$ accuracy, $0.5=$ sharing $)$, a news type dummy $(-0.5=$ false, $0.5=$ true $)$, a political concordance dummy $(-0.5=$ discordant, $0.5=$ concordant), and all 2-way and 3-way interactions. [Political concordance is defined based on the match between content and ideology. Specifically, political concordant = Pro-Democratic [Pro-Republican] news (based on a pretest) for American individuals who prefer the Democratic [Republican] party over the Republican [Democratic]. Politically discordant is the opposite.] Our key prediction was that there would be a negative interaction between condition and news type, such that the difference between false and true is smaller in the sharing condition than the accuracy condition. A secondary prediction was that there would be a positive interaction between condition and concordance, such that the difference between concordant and discordant is larger in the sharing condition than the accuracy condition. We also said we would check for a 3-way interaction, and use a Wald test of the relevant net coefficients to test how sharing likelihood of false concordant headlines compares to true discordant headlines. Finally, as robustness checks, we said we would repeat the main analysis using logistic regression instead of linear regression, and using ratings that are z-scored within condition.

\section{Study 2}

Study 2 extended Study 1's observation that most people self-report that it is important to not share accuracy information on social media. First, Study 2 assesses the relative importance placed on accuracy by also asking about the importance of various other factors. Second, Study 2 tested whether Study 1's results would generalize beyond MTurk by recruiting participants from Lucid for Academics, delivering a sample that matches the distribution of American residents on age, gender, ethnicity, and geographic region. Third, Study 2 avoided the potential spillover effects demonstrated in Extended Data Figure 1 by not having participants complete a task related to social media beforehand.

In total, 401 participants (Mean age $=43.7$ ) completed the survey on January $9^{\text {th }}-12^{\text {th }}, 2020$, including 209 males and 184 females, and 8 indicating other gender identities. Participants were asked "When deciding whether to share a piece of content on social media, how important is it to you that the content is..." and then were given a response grid where the columns were labeled "Not at all", "Slightly", "Moderately", "Very", and "Extremely", and the rows were labeled "Accurate", "Surprising", "Interesting", "Aligned with your politics", and "Funny".

This study was approved by the MIT COUHES (Protocol \#1806400195).

\section{Studies 3, 4, and 5}

In Studies 3, 4, and 5 we investigate whether shifting attention to accuracy increases the veracity of the news people are willing to share. In particular, participants were asked to judge the accuracy of a single (politically neutral) news headline at the beginning of the study, ostensibly as part of a pretest for another study. We then tested whether this subtle accuracy-cue impacts 
individuals' ability to discern between false and true news when making judgments about social media sharing. The principal advantage of this design is that the manipulation is subtle and not explicitly linked to the main task. Thus, although social desirability bias may lead people to underreport their likelihood of sharing misinformation overall, it is unlikely that any betweencondition difference is driven by participants believing that the accuracy question at the beginning of the treatment condition was designed to make them take accuracy into account when making sharing decisions during the main experiment. It is therefore relatively unlikely that any treatment effect on sharing would be due to demand characteristics or social desirability.

The only difference between Studies 3 and 4 was the set of headlines used, to demonstrate the generalizability of these findings. Study 5 used a more representative sample and included an active control condition and a second treatment condition that primed accuracy concerns in a different way. Studies 3 and 4 were approved by the Yale University Committee for the Use of Human Subjects (IRB protocol \#1307012383). Study 5 was approved by the University of Regina Research Ethics Board (Protocol \#2018-116).

\section{Participants}

In Study 3, we preregistered a target sample of 1,200 participants from MTurk. In total, 1,254 participants began the survey between October $4^{\text {th }}-6^{\text {th }}, 2017$. However, 21 participants reporting not having a Facebook profile at the outset of the study and, as per our preregistration, were not allowed to proceed; and 71 participants did not complete the survey. The full sample (Mean age $=33.7$ ) included 453 males, 703 females, and 2 who did not answer the question. Following the main task, participants were asked if they "would ever consider sharing something political on Facebook" and were given the following response options: 'Yes', 'No', and 'I don't use social media'. As per our preregistration, only participants who selected 'Yes' to this question were included in our main analysis. This excluded 431 people and the sample of participants who would consider sharing political content (Mean age $=34.5$ ) included 274 males, 451 females, and 2 who did not answer the gender question. Unlike in Study 1, because this question was asked after the experimental manipulation (rather than at the outset of the study), there is the possibility that this exclusion may introduce selection effects and undermine causal inference ${ }^{35}$. While there was no significant difference in responses to this political sharing question between conditions in any of the three accuracy priming experiments $\left(\chi^{2}\right.$ test; S3: $\chi^{2}(1, N=1,158)=.156$, $\left.p=.69 ; \mathrm{S} 4: \chi^{2}(1, N=1,248)=.988, p=.32 ; \mathrm{S} 5, \chi^{2}(3, N=1,287)=2.320, p=.51\right)$, for completeness we show that all of our results are robust to including all participants.

In Study 4, we preregistered a target sample of 1,200 participants from MTurk. In total, 1,328 participants began the survey between November $28^{\text {th }}-30^{\text {th }}$, 2017. However, 8 participants did not report having a Facebook profile and 72 participants did not finish the survey. The full sample (Mean age $=33.3$ ) included 490 males, 757 females, and 1 who did not answer the question. Restricting to participants were responded "Yes" when asked if they "would ever consider sharing something political on Facebook" excluded 468 people, such that the sample of participants who would consider sharing political content (Mean age $=33.6$ ) included 282 males, 497 females, and 1 who did not answer the gender question. 
634 In Study 5, we preregistered a target sample of 1,200 participants from Lucid. In total, 1,628 participants began the survey between April $30^{\text {th }}$ - May $1^{\text {st }}, 2019$. However, 236 participants reported not having a Facebook profile (and thus were not allowed to complete the survey) and 105 participants did not finish the survey. The full sample (Mean age $=45.5$ ) included 626 males and 661 females. Restricting to participants were responded "Yes" when asked if they "would ever consider sharing something political on Facebook" excluded 616 people, such that the sample of participants who would consider sharing political content (Mean age $=44.3$ ) included 333 males and 338 females.

\section{Materials}

In Study 3, we presented participants with 24 news headlines from ${ }^{19}$; in Study 4, we presented participants with a different set of 24 news headlines selected via pretest; and in Study 5, we presented participants with yet another set of 20 news headlines selected via pretest. In all studies, half of the headlines were false (selected from a third-party fact-checking website, Snopes.com, and therefore verified as being fabricated and untrue) and the other half were true (accurate and selected from mainstream news outlets to be roughly contemporary with the false news headlines). Moreover, half of the headlines were Pro-Democratic/Anti-Republican and the other half were Pro-Republican/Anti-Democrat (as determined by the pretests). See SI Section 1 for further details on the pretests.

As in Study 1, following the main task participants in Studies 3-5 were asked about the importance of only sharing accurate news articles on social media (Study 4 also asked about the important participants' friends placed on only sharing accurate news on social media). Participants then completed various exploratory measures and demographics. The demographics included the question "If you absolutely had to choose between only the Democratic and Republican party, which would do you prefer?" followed by the following response options: Democratic Party, Republican Party. We use this question to classify participants as Democrats versus Republicans.

\section{Procedure}

In all three studies, participants were first asked if they have a Facebook account and those who did not were not permitted to complete the study. Participants were then randomly assigned to one of two conditions in Studies 3 and 4, and one of four conditions in Study 5.

In the Treatment condition of all three studies, participants were instead given the following instructions: "First, we would like to pretest an actual news headline for future studies. We are interested in whether people think it is accurate or not. We only need you to give your opinion about the accuracy of a single headline. We will then continue on to the primary task. Note: The image may take a moment to load." Participants were then shown a politically neutral headline and were asked: "To the best of your knowledge, how accurate is the claim in the above headline?" and were given the following response scale: "Not at all accurate, Not very accurate, Somewhat accurate, Very accurate." One of two politically neutral headlines ( 1 true, 1 false) was randomly selected in Studies 3 and 4; one of four politically neutral headlines (2 true, 2 false) was randomly selected in Study 5 . 
In the Active Control condition of Study 5, participants were told: "First, we would like to pretest an actual news headline for future studies. We are interested in whether people think it is funny or not. We only need you to give your opinion about the funniness of a single headline. We will then continue on to the primary task. Note: The image may take a moment to load." They were then presented with one of the same four neutral news headlines used in the Treatment and asked: "In your opinion, is the above headline funny, amusing, or entertaining?" (response options: Extremely unfunny, moderately unfunny, slightly unfunny, slightly funny, moderately funny, extremely funny).

In the Importance Treatment condition of Study 5, participants were asked the following question at the outset of the study: "Do you agree or disagree that 'it is important to only share news content on social media that is accurate and unbiased'?" (Response options: strongly agree to strongly disagree).

Participants in all conditions were then told: "You will be presented with a series of news headlines from 2016 and 2017 (24 in total) [2017 and 2018 (20 in total) for Study 5]. We are interested in whether you would be willing to share the story on Facebook. Note: The images may take a moment to load." They then proceeded to the main task in which they were presented with the true and false headlines and for each were asked "If you were to see the above article on Facebook, how likely would you be to share it" and given the following response scale:

"Extremely unlikely, Moderately unlikely, Slightly unlikely, Slightly likely, Moderately likely, Extremely likely". We used a continuous scale, instead of the binary scale used in Study 1, to increase the sensitivity of the measure.

\section{Analysis plan}

Our preregistrations specified that all analyses would be performed at the level of the individual item (i.e., one data point per item per participant, with the 6-point sharing Likert scale rescaled to the interval $[0,1]$ ) using linear regression with robust standard errors clustered on participant.

However, we subsequently realized that we should also be clustering standard errors on headline (as multiple ratings of the same headline are non-independent in a similar way to multiple ratings from the same participant), and thus deviated from the preregistrations in this minor way (all key results are qualitatively equivalent if only clustering standard errors on participant).

In Studies 3 and 4, the key preregistered test was an interaction between a condition dummy $(0=$ Control, $1=$ Treatment $)$ and a news veracity dummy $(0=$ False, $1=$ True $)$. This was to be followed-up by tests for simple effects of news veracity in each of the two conditions; and, specifically, the effect was predicted to be larger in the Treatment condition. We also planned to test for simple effects of condition for each of the two types of news; and, specifically, the effect was predicted to be larger for false relative to true news. We also conducted a post hoc analysis using a linear regression with robust standard errors clustered on participant and headline to examine the potential moderating role of a dummy for the participant's partisanship (preference for the Democratic versus Republican party) and a dummy for the headline's ideological concordance (Pro-Democratic [Pro-Republican] headlines scored as concordant for participants who preferred the Democratic [Republican] party; Pro-Republican [Pro-Democratic] headlines 
scored as discordant for participants who preferred the Democratic [Republican] party). For ease of interpretation, we z-scored the partisanship and concordance dummies, and then included all possible interactions in the regression model. To maximize statistical power for these moderation analyses, we pooled the data from Studies 3 and 4.

In Study 5, the first preregistered test was to compare whether the active and passive control conditions differed, by testing for significant a main effect of condition $(0=$ passive, $1=$ active $)$, or significant interaction between condition and news veracity $(0=$ fake, $1=$ real $)$. If these did not differ, we preregistered that we would combine the two control conditions for subsequent analyses. We would then test whether the two treatment conditions differ from the control condition( $\mathrm{s})$ by testing for an interaction between dummies for each treatment $(0=$ passive or active control, $1=$ treatment being tested) and news veracity. This was to be followed-up by tests for simple effects of news veracity in each of the conditions; and, specifically, the effect was predicted to be larger in the treatment conditions. We also planned to test for simple effects of condition for each of the two types of news; and, specifically, the effect was predicted to be larger for false relative to true news.

\section{Study 6}

Studies 3, 4, and 5 found that a subtle reminder of the concept of accuracy decreased sharing of false (but not true) news. In Study 6, we instead use a Full Attention Treatment that directly forces participants to consider the accuracy of each headline before deciding whether to share it. This allows us to determine - within this particular context - the maximum effect that can be obtained by focusing attention on accuracy. Furthermore, using the accuracy ratings elicited in the Full Attention Treatment, we can also determine what fraction of shared content was believed to be accurate versus inaccurate by the sharer. Together, these analyses allow us to infer the fraction of sharing of false content that is attributable to inattention, confusion about veracity, and purposeful sharing of falsehood.

This study was approved by the Yale University Committee for the Use of Human Subjects (IRB protocol \#1307012383).

\section{Participants}

We combine two rounds of data collection on MTurk, the first of which had 218 participants begin the study on August 11 $1^{\text {th }}, 2017$, and the second of which had 542 participants begin the study on August $24^{\text {th }}, 2017$, for a total of 760 participants. However, 14 participants did not report having a Facebook profile and 33 participants did not finish the survey. The full sample (Mean age $=34.0$ ) included 331 males, 376 females, and 4 who did not answer the question. Participants were asked if they "would ever consider sharing something political on Facebook" and were given the following response options: Yes, No, I don't use social media. Only participants who selected 'Yes' to this question were included in our main analysis, as in our other studies (there was no significant difference in responses between conditions, $\chi^{2}(2)=1.07$, $p=0.585$ ). This excluded 313 people and the final sample (Mean age $=35.2$ ) included 181 males, 213 females, and 4 who did not answer the gender question. For robustness, we also report analyses including all participants. 


\section{Materials}

We presented participants with the same 24 headlines used in Study 3.

\section{Procedure}

Participants were first asked if they have a Facebook account and those who did not were not permitted to complete the study. Participants were then randomly assigned to one of two conditions. In the Full Attention Treatment condition, participants were given the following instructions: "You will be presented with a series of news headlines from 2016 and 2017 (24 in total). We are interested in two things: 1) Whether you think the headlines are accurate or not. 2) Whether you would be willing to share the story on Facebook. Note: The images may take a moment to load." In the Control condition, participants were told: "You will be presented with a series of news headlines from 2016 and 2017 (24 in total). We are interested in whether you would be willing to share the story on Facebook. Note: The images may take a moment to load." Participants in both conditions were asked "If you were to see the above article on Facebook, how likely would you be to share it" and given the following response scale: "Extremely unlikely, Moderately unlikely, Slightly unlikely, Slightly likely, Moderately likely, Extremely likely". Crucially, in the Treatment condition, prior to being asked the social media sharing question, participants were asked: "To the best of your knowledge, how accurate is the claim in the above headline?" and given the following response scale: "Not at all accurate, Not very accurate, Somewhat accurate, Very accurate."

Analysis

The goal of our analyses is the estimate what fraction of sharing of false headlines is attributable to confusion (incorrectly believing the headlines are accurate), inattention (forgetting to consider the headlines' accuracy; as per the inattention-based account), and purposeful sharing of false content (as per the preference-based account). We can do so by utilizing the sharing intentions in both conditions, and the accuracy judgments in the Full Attention Treatment (no accuracy judgments were collected in the control). Because participants in the Full Attention Treatment are forced to consider the accuracy of each headline before deciding whether they would share it, inattention to accuracy is entirely eliminated in the Full Attention Treatment. Thus, the difference in sharing of false headlines between Control and Full Attention Treatment indicates the fraction of sharing in Control that was attributable to inattention. We can then use the accuracy judgments to determine how much of the sharing of false headlines in the Full Attention Treatment was attributable to confusion (indicated by the fraction of shared headlines that participants rated as accurate) versus purposeful sharing (indicated by the fraction of shared headlines that participants rated as inaccurate).

Concretely, we do the analysis as follows. First, we dichotomize responses, classifying sharing intentions of "Extremely unlikely", "Moderately unlikely", and "Slightly unlikely" as "Unlikely to share" and "Slightly likely", "Moderately likely", and "Extremely likely" as "Likely to share"; and classifying accuracy ratings of "Not at all accurate" and "Not very accurate" as "Not 
accurate" and "Somewhat accurate" and "Very accurate" as "Accurate". Then we define the fraction of sharing of false content due to each factor as follows:

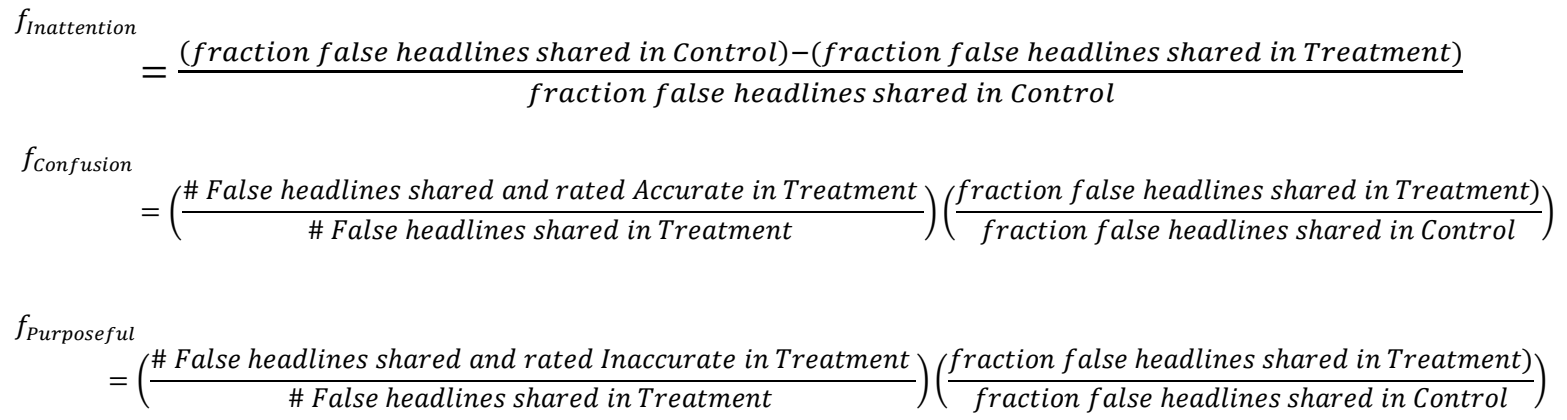

For an intuitive visualization of these expressions, see Figure $2 \mathrm{~d}$.

To calculate confidence intervals on our estimates of the relative impact of inattention, confusion, and purposeful sharing, we use bootstrapping simulations. We create 10,000 bootstrap samples by sampling with replacement at the level of the subject. For each sample, we calculate the difference in fraction of sharing of false information explained by each of the three factors (i.e. the three pairwise comparisons). We then determine a two-tailed $p$-value for each comparison by doubling the fraction of samples in which the factor that explains less of the sharing in the actual data is found to explain more of the sharing.

\section{Preregistration}

Although we did complete a preregistration in connection with this experiment, we do not follow it here. The analyses we preregistered simply tested for an effect of the manipulation on sharing discernment, as in Studies 3-5. After conducting the experiment, we realized that we could analyze the data in an alternative way to gain insight into the relevant impact of the three reasons for sharing misinformation described in this paper. It is these (post hoc) analyses that we focus on in the current paper. Importantly, Extended Data Table 2 shows that equivalent results are obtained when analyzing the two samples separately (the first being a pilot for the pre-registered experiment, and the second being the pre-registered experiment), helping to address the post hoc nature of these analyses.

\section{Study 7}

In Study 7 we set out to test whether the results of the survey experiments in Studies 3-5 would generalize to real sharing decisions "in the wild", and to misleading but not blatantly false news. Thus, we conducted a digital field experiment on Twitter in which we delivered the same intervention from the Treatment condition of the survey experiments to users who had previously shared links to unreliable news sites. We then examined the impact of receiving the intervention on the quality of the news they subsequently shared. The experiment was approved by Yale University Committee of the Use of Human Subjects IRB protocol \#2000022539 and MIT COUHES Protocol \#1806393160. While all analysis code is posted online, we did not publicly post the data due to privacy concerns (even with de-identified data, it is likely possible to back 
out which Twitter user corresponds with many of the users in the dataset). Researchers interested in accessing the data are asked to contact the corresponding author.

Study 7 is an aggregation of three different waves of data collection, the details of which are summarized in Extended Data Table 3. (These are all of the data we collected - nothing was left "in the file drawer"; and the decision to conclude data collection was made prior to running any of the analyses reported in this paper.)

\section{Participants}

The basic experimental design involved sending a private direct message (DM) to users asking them to rate the accuracy of a headline (as in the Treatment condition of the survey experiments). Twitter only allows DMs to be sent from account X to account $Y$ if account $Y$ follows account X. Thus, our first task was to assemble a set of accounts with a substantial number of followers (who we could then send DMs to). In particular, we needed followers who were likely to share misinformation. Our approach was as follows.

First, we created a list of tweets with links to one of two news sites that professional fact-checkers rated as extremely untrustworthy ${ }^{25}$ but that are nonetheless fairly popular: Breitbart.com and infowars.com. We identified these tweets by (i) retrieving the timeline of the Breitbart Twitter account using the Twitter REST API (Infowars has been banned from Twitter and thus has no Twitter account) and (ii) searching for tweets containing a link to the corresponding domain using the Twitter advanced search feature and either collecting the tweet IDs manually (wave 1) or via scraping (waves 2 and 3). Next, we used the Twitter API to retrieve lists of users who retweeted each of those tweets (we periodically fetched the list of "retweeters" since the Twitter API only provides the last 100 users "retweeters" of a given tweet). As shown in Table S9, across the three waves this process yielded a potential participant list of 136,379 total Twitter users with some history of retweeting links to misleading news sites.

Next, we created a series of accounts with innocuous names (e.g. "CookingBot"); we created new accounts for each experimental wave. Each of the users in the potential participant list was then randomly assigned to be followed by one of our accounts. We relied on the tendency of Twitter users to reciprocally follow-back to create our set of followers. Indeed, $8.3 \%$ of the users that were followed by one of our accounts chose to follow our account back. This yielded a total of 11,364 followers across the three waves. (After the completion of our experiments, Twitter has made it substantially harder to follow large numbers of accounts without getting suspended, which creates a challenge for using this approach in future work; a solution is to use Twitter's targeted advertising to target ads whose goal is the accruing of followers at the set of users one would like to have in one's subject pool.)

To determine eligibility and to allow blocked randomization, we then identified (i) users' political ideology using the algorithm from Barberá, Jost, Nagler, Tucker, and Bonneau (2015); (ii) their probability of being a bot, using the bot-or-not algorithm ${ }^{37}$; (iii) the number of tweets to one of the 60 websites with fact-checker ratings that will form our quality measure; and (iv) the average fact-checker rating (quality score) across those tweets. 
For waves 1 and 2, we excluded users who tweeted no links to any of the 60 sites in our list in the two weeks prior to the experiment; who could not be given an ideology score; who could not be given a bot score; or who had a bot score above 0.5 (in wave 1, we also excluded a small number of very high-frequency tweeters for whom we were unable to retrieve all relevant tweets due to the 3200-tweet limit of the Twitter API). In wave 3, we took a different approach to avoiding bots, namely avoiding high-frequency tweeters. Specifically, we excluded participants who tweeted more than 30 links to one of the 60 sites in our list in the two weeks prior to the experiment, as well as excluding those who tweeted fewer than 5 links to one of the 60 sites (to avoid lack of signal). This resulted in a total of 5,379 unique Twitter users across the three waves. (Note that these exclusions were applied ex ante, and excluded users were not included in the experiment, rather than implementing post hoc exclusions.)

One might be concerned about systematic differences between the users we included in our experiments versus those who we followed but either did not follow us back or we excluded prior to the experiment beginning. To gain some insight into this question, we compared the characteristics of the 5,379 users in our experiment to a random sample of 10,000 users that we followed but did follow us back (sampled proportional to the number of users in each wave). For each user we retrieved number of followers, number of accounts followed, number of favorites, and number of tweets. We also estimated political ideology as per Barberá, Jost, Nagler, Tucker, and Bonneau (2015), probability of being a bot (bot or not; ${ }^{37}$ ), and age and gender using based on profile pictures using the Face Plus Plus algorithm ${ }^{38-40}$. Finally, we checked whether the account had been suspended or deleted. As shown in Extended Data Figure 5, relative to users who did not follow us back, the users that wound up in our experiment followed more accounts, had more followers, favorited more tweets, were more conservative, were older, and were more likely to be bots $(p<.001$ for all); and were more likely to have had their accounts suspended or deleted $(p=.012)$. These observations suggest that to the extent that our recruitment process induced selection, it is in a direction that works against the effectiveness of our treatment: the users in our experiment are likely to be less receptive to the intervention than users more generally, and therefore our effect size is likely an underestimate of the effect we would have observed in the full sample.

\section{Materials \& procedure}

The treatment in Study 7 was very similar to the survey experiments: Users were sent a DM asking them to rate the accuracy of a single non-political headline (see Figure 4b). An advantage of our design is that this DM is coming from an account that the user has themselves opted in to following, rather than from a totally unknown account. Furthermore, the DM begins by saying "Thanks for following me!" and sending such thank-you DMs is a common practice on Twitter. These factors should substantially mitigate any possibility of the users feeling suspicious or like they are being surveilled by our account, and instead make the DM feel like a typical interaction on Twitter.

We did not expect users to respond to our message - our intervention was based on the idea that merely reading the opening line ("How accurate is this headline?") would make the concept of accuracy more salient. And because we could not reliably observe whether (or when) users read the message, we performed intent-to-treat analyses that included all subjects. Furthermore, to avoid demand effects, users were not informed that the message was being sent as part of a research 
study, and the accounts from which we sent the messages had innocuous descriptions (e.g., "Cooking Bot"). Not informing users about the study was essential for ecological validity, and we felt that the scientific and practical benefits justified this approach given that the potential harm to participants was minimal, and the tweet data were all publicly available. See SI Section 4 for more discussion on the ethics of digital field experimentation.

Because of DM rate limits imposed by Twitter, we could only send DMs to roughly 20 users per account per day. Thus, we conducted each wave in a series of 24-hour blocks in which a small subset of users was DM'd on each day. All tweets and retweets posted by all users in the experiment were collected on each day of the experiment. All links in these tweets were extracted (including expanding shortened URLs). The dataset was then composed of the subset of these links that linked to one of 60 sites whose trustworthiness had been rated by professional fact-checker in prior work $^{25}$ (with the data entry being the trust score of the linked site).

To allow for causal inference, we used a randomized roll-out (also called stepped-wedge) design in which users were randomly assigned to a treatment date. This allows us to analyze all tweets made during all of the 24-hour treatment blocks, comparing tweets from users who received the $\mathrm{DM}$ at the start of a given block (Treated) to tweets from users who had not yet been DM'd (Control). Because treatment date is randomly assigned, it can be inferred that any systematic difference revealed by this comparison was caused by the treatment. (Wave 2 also included a subset of users who were randomly assigned to never receive the DM.) To improve the precision of our estimate, random assignment to treatment date was approximately balanced across bot accounts in all waves, and across political ideology, number of tweets to rated sites in the two weeks before the experiment, and average quality of those tweets across treatment dates in waves 2 and 3.

Because our treatment was delivered via the Twitter API, we were vulnerable to unpredictable changes to, and unstated rules of, the API. These gave rise to several deviations from our planned procedure. On day 2 of wave 1, fewer than planned DMs were sent as our accounts were blocked part way thru the day; and no DMs were sent on day 3 of wave 1 (hence, that day is not included in the experimental dataset). On day 2 of wave 2, Twitter disabled the DM feature of the API for the day, so we were unable to send the DMs in an automated fashion as planned. Instead, all 370 DMs sent on that day were sent manually over the course of several hours (rather than simultaneously). On day 3 of wave 2, the API was once again functional, but partway through sending the DMs, the credentials for our accounts were revoked and no further DMs were sent. As a result, only 184 of the planned 369 DMs were sent on that day. Furthermore, because we did not randomize the order of users across stratification blocks, the users on day 3 who were not DM'd were systematically different from those who were DM'd. (As discussed in detail below, we consider analyses that use an intent-to-treat approach for wave 2 day 3 - treating the data as if all 369 DMs had indeed been sent - as well as analyses that exclude the data from wave 2 day 3.)

\section{Analysis plan}

As the experimental design and the data were substantially more complex than the survey experiment studies and we lacked well-established models to follow, it was not straightforward to determine the optimal way to analyze the data in Study 7. This is reflected, for example, in the 
998 999

1000

1001

1002

1003

1004

1005

1006

1007

1008

1009

1010

1011

1012

1013

1014

1015

1016

1017

1018

1019

1020

1021

1022

1023

1024

1025

1026

1027

1028

1029

1030

1031

1032

1033

1034

1035

1036

1037

1038

1039

1040

1041

1042

1043

fact that wave 1 was not preregistered, two different preregistrations were submitted for wave 2 (one prior to data collection and one following data collection but prior to analyzing the data), and one preregistration was submitted for wave 3 , and each of the preregistrations stipulated a different analysis plan. Moreover, after completing all three waves, we realized that all of the analyses proposed in the preregistrations do not actually yield valid causal inferences because of issues involving missing data (as discussed in more detail below in the "Dependent variable" section). Therefore, instead of conducting a particular preregistered analysis, we consider the pattern of results across a range of reasonable analyses.

All analyses are conducted at the user-day level using linear regression with heteroscedasticityrobust standard errors clustered on user. All analyses include all users on a given day who have not yet received the DM as well as users who received the DM on that day (users who received the DM more than 24 hours before the given day are not included). All analyses use a posttreatment dummy $(0=$ user has not yet been DM'd, $1=$ user received the DM that day) as the key independent variable. We note that this is an intent-to-treat approach that assumes that all DMs on a given day are sent at exactly the same time, and counts all tweets in the subsequent 24-hour block as post-DM. Thus, to the extent that technical issues caused tweets on a given day to be sent somewhat earlier or later than the specified time, this approach may somewhat underestimate the treatment effect.

The analyses we consider differ in the following ways: dependent variable, model specification, type of tweets considered, approach to handling randomization failure, and approach to determining statistical significance. We now discuss each of these dimensions in more detail.

1. Dependent variable: We consider three different ways of quantifying tweet quality. Across approaches, a key issue is how to deal with missing data. Specifically, on days when a given user does not tweet any links to rated sites, the quality of their tweeted links is undefined. The approach implied in our preregistrations was to simply omit missing userdays (or to conduct analyses at the level of the tweet). Because the treatment is expected to influence the probability of tweeting, however, omitting missing user-days has the potential to create selection and thus undermine causal inference (and tweet-level analyses are even more problematic). For example, if a user tweets as a result of being treated but would not have tweeted had they been in the control (or does not tweet as a result of treatment but would have tweeted have they been in the control), then omitting the missing user-days breaks the independence between treatment and potential outcomes ensured by random assignment. Given that only $47.0 \%$ of user-days contained at least one tweeted link to a rated site, such issues are potentially quite problematic. We therefore consider three approaches to tweet quality that avoid this missing data problem.

The first measure is the average relative quality score. This measure assigns each tweeted link a relative quality score by taking Pennycook $\&$ Rand's fact-checker trust rating (quality score, $[0,1]$ ) of the domain ${ }^{(24)}$ being linked to, and subtracting the baseline quality score of 0.34 (this corresponds to the average quality score of all pre-treatment tweets across all users in all of the experimental days of Study 4). Each user-day is then assigned an average relative quality score by averaging the relative quality score of all tweets made by the user in question on the day in question; and users who did not tweet on a given day 
are assigned an average relative quality score of 0 (thus avoiding the missing data problem). The average relative quality score is thus defined over the interval $[-0.34,0.66]$. Importantly, this measure is quite conservative because the (roughly half of) post-treatment user-days where data is missing are scored as $0 \mathrm{~s}$. Thus, this measure assumes that the treatment had no effect on users who did not tweet on the treatment day. If, instead, nontweeting users would have shown the same effect had they actually tweeted, the estimated effect size would be roughly twice as large as what we observe here. We note that this measure is equivalent to using average quality scores (rather than relative quality score) and imputing the baseline quality score to fill missing data (so assuming that on missing days, the user's behavior matches the subject pool average).

The second measure is the summed relative quality score. This measure assigns each tweeted link a relative quality score in the same manner described above. A given userday's summed relative quality score is then 0 plus the sum of the relative quality scores of each link tweeted by that user on that day. Thus, the summed relative quality score increases as a user tweets more and higher quality links, and decreases as the user tweets more and lower quality links; and, as for the average relative quality score, users who tweet no rated links received a score of 0 . As this measure is unbounded in both the positive and negative directions, and the distribution contains extreme values in both directions, we winsorize summed relative quality scores by replacing values above the $95^{\text {th }}$ percentile with the $95^{\text {th }}$ percentile, and replacing values below the $5^{\text {th }}$ percentile with values below the $5^{\text {th }}$ percentile (our results are qualitatively robust to alternative choices of threshold at which to winsorize).

The third measure is discernment, or the difference in the number of links to mainstream sites versus misinformation sites shared on a given user-day. This measure is mostly closely analogous to the analytic approach taken in Studies 2-4. To assess the impact of the intervention on discernment, we transform the data into long format such that there are two observations per user-day, one indicating the number of tweets to mainstream sites and the other indicating the number of tweets to misinformation sites (as defined in Pennycook \& Rand, 2019). We then include a source type dummy $(0=$ misinformation, $1=$ mainstream $)$ in the regression, and interact this dummy with each independent variable. The treatment increases discernment if there is a significant positive interaction between the posttreatment dummy and the source type dummy. As these count measures are unbounded in the positive direction, and the distributions contain extreme values, we winsorize by replacing values above the $95^{\text {th }}$ percentile of all values with the $95^{\text {th }}$ percentile of all values (our results are qualitatively robust to alternative choices of threshold at which to winsorize).

Finally, as a control analysis, we also consider the treatment effect on the number of tweets in each user-day that did not contain links to any of the 60 rated news sites. As this count measure is unbounded in the positive direction, and the distribution contains extreme values, we winsorize by replacing values above the $95^{\text {th }}$ percentile of all values with the $95^{\text {th }}$ percentile of all values (our results are qualitatively robust to alternative choices of threshold at which to winsorize). 
2. Determining statistical significance: We consider the results of two different methods for computing $\mathrm{p}$-values for each model. The first is the standard regression approach, in which robust standard errors clustered on user are used to calculate $p$-values. The second employs Fisherian Randomization Inference (FRI) to compute a p-value that is exact (i.e., has no more than the nominal Type I error rate) in finite samples ${ }^{26,41-43}$. FRI is non-parametric and thus does not require any modeling assumptions about potential outcomes. Rather, the stochastic assignment mechanism determined by redrawing the treatment schedule, exactly as done in the original experiment, determines the distribution of the test statistic under the null hypothesis ${ }^{43}$. Based on our stepped-wedge design, our treatment corresponds to the day on which the user receives the DM. Thus, to perform FRI, we create over 10,000 permutations of the assigned treatment day for each user by re-running the random assignment procedure used in each wave, and recompute the t-statistic for the coefficient of interest in each model in each permutation. We then determine $p$-values for each model by computing the fraction of permutations that yielded t-statistics with absolute value larger than the t-statistic observed in the actual data. Note that therefore, FRI takes into account the details of the randomization procedure that approximately balanced treatment date across bots in all waves, and across ideology, tweet frequency, and tweet quality in waves 2 and 3.

3. Model specification: We consider four different model specifications. The first includes wave dummies. The second post-stratifies on wave by interacting centered wave dummies with the post-treatment dummy. This specification also allows us to assess whether any observed treatment effect significantly differs across waves by performing a joint significance test on the interaction terms. The third includes date dummies. The fourth post-stratifies on date by interacting centered date dummies with the post-treatment dummy. (We note that the estimates produced by the first two specifications may be problematic if there are secular trends in quality and they are used in conjunction with linear regression rather than FRI, but we include them for completeness; excluding them does not qualitatively change our conclusions.)

4. Tweet type: The analysis can include all tweets, or can focus only on cases where the user retweets the tweet containing the link without adding any comment. The former approach is more inclusive, but may contain cases in which the user is not endorsing the shared link (e.g., someone debunking an incorrect story may still link to the original story). Thus, the latter case might more clearly identify tweets that are uncritically sharing the link in question. More importantly, retweeting without comment (low-engagement sharing) exemplifies the kind of fast, low-attention action that is our focus (wherein we argue that people share misinformation despite a desire to only share accurate information - because the attentional spotlight is focused on other content dimensions). Primary tweets are much more deliberate actions, ones in which it is more likely that the user did consider their action before posting (and thus where our accuracy nudge would be expected to be ineffective).

5. Article type: The analysis can include all links, or can exclude (as much as possible) links to opinion articles. While the hyperpartisan and fake news sites in our list do not typically demarcate opinion pieces, nearly all of the mainstream sites include "opinion" in the URL 
of opinion pieces. Thus, for our analyses that minimize opinion articles, we exclude the $3.5 \%$ of links (6.8\% of links to mainstream sources) that contained "/opinion/" or "/opinions/" in the URL.

6. Approach to randomization failure: As described above, due to issues with the Twitter API on day 3 of wave 2, there was a partial randomization failure on that day (many of the users assigned to treatment received no DM). We consider two different ways of dealing with this randomization failure. In the intent-to-treat approach, we include all users from the randomization-failure day (with the post-treatment dummy taking on the value 1 for all users who were assigned to be DM'd on that day, regardless of whether they actually received a DM). In the exclusion approach, we instead drop all data from that day.

In the main text, we present the results of the specification in which we analyze retweets without comment, include links to both opinion and non-opinion articles, include wave fixed effects, calculate $p$-values using FRI, and exclude data from one day on which a technical issue led to a randomization failure. Extended Data Table 4 presents the results of all specifications.

The primary tests of effects of the treatment compare differences in tweet quality for all eligible user-days. However, this includes many user-days for which there are no tweets to rated sites, which can occur, for example, because that user does not even log on to Twitter on that day. To quantify effect sizes on a more relevant subpopulation, we employ the principal stratification framework whereby each unit belongs to one of four latent type ${ }^{27,28}$ : never-taker user-days (which would not have any rated tweets in either treatment or control), always-taker user-days (user-days where the user tweets rated links that day in both treatment and control), complier user-days (where treatment causes tweeting of rated links that day, which would not have occurred otherwise), and defier user-days (where treatment prevents tweeting of rated links). Since the estimated treatment effects on whether a user tweets on a given day are mostly positive (although not statistically significant; see SI Table S9), we assume the absence of defier userdays. Under this assumption, we can estimate the fraction of user-days that are not never-taker user-days (i.e., are complier or always-taker user-days). This is then the only population on which treatment effects on rated tweet quality can occur, as the never-taker user-days are by definition unaffected by treatment with respect to rated tweets. We can then estimate treatment effects on quality and discernment on this possibly affected subpopulation by rescaling the estimates for the full population by dividing by the estimated fraction of non-never-taker userdays. These estimates are then larger in magnitude since they account for the dilution due to the presence of units that are not affected by treatment since they do not produce tweets whether in treatment or control.

Moreover, it is important to remember that our estimates of the effect size for our subtle, one-off treatment are conservative: While our intent-to-treat approach necessarily assumes that the message was seen immediately - and thus counts all tweets in the 24 hours after the message was sent as "treated" - we cannot reliably tell when (or even if) any given user saw our message. Thus, 
1179 it is likely that many of the tweets we are counting as post-treatment were not actually treated, and

that we are underestimating the true treatment effect as a result.

\section{Additional References (Methods)}

33. Watson, D., Clark, L. A. \& Tellegen, A. Development and Validation of Brief Measures of Positive and Negative Affect - the Panas Scales. J. Pers. Soc. Psychol. 54, 1063-1070 (1988).

34. Mosleh, M., Pennycook, G. \& Rand, D. Self-reported willingness to share political news articles in online surveys correlates with actual sharing on Twitter. PLoS One 15, e0228882 (2020).

35. Montgomery, J. M., Nyhan, B. \& Torres, M. How Conditioning on Posttreatment Variables Can Ruin Your Experiment and What to Do about It. Am. J. Pol. Sci. 62, 760-775 (2018).

36. Barberá, P., Jost, J. T., Nagler, J., Tucker, J. A. \& Bonneau, R. Tweeting From Left to Right: Is Online Political Communication More Than an Echo Chamber? Psychol. Sci. 26, 1531-1542 (2015).

37. Davis, C. A., Varol, O., Ferrara, E., Flammini, A. \& Menczer, F. BotOrNot: A System to Evaluate Social Bots. in Proceedings of the 25th International Conference Companion on World Wide Web 273-274 (Association for Computing Machinery (ACM), 2016). doi:10.1145/2872518.2889302

38. Chakraborty, A. et al. Who Makes Trends? Understanding Demographic Biases in Crowdsourced Recommendations. Proc. 11th Int. Conf. Web Soc. Media, ICWSM 2017 22-31 (2017).

39. Kteily, N. S., Rocklage, M. D., McClanahan, K. \& Ho, A. K. Political ideology shapes the amplification of the accomplishments of disadvantaged vs. Advantaged group members. Proc. Natl. Acad. Sci. U. S. A. 116, 1559-1568 (2019).

40. An, J. \& Weber, I. \#greysanatomy vs. \#yankees: Demographics and Hashtag Use on Twitter. AAAI Conf. Web Soc. Media Icwsm 523-526 (2016).

41. Rubin, D. B. Randomization Analysis of Experimental Data: The Fisher Randomization Test Comment. J. Am. Stat. Assoc. 75, 591 (1980).

42. Rosenbaum, P. R. Overt bias in observational studies. in Observational Studies 71-104 (Springer New York, 2002). doi:10.1007/978-1-4757-3692-2

43. Imbens, G. W. \& Rubin, D. B. Causal inference: For statistics, social, and biomedical sciences an introduction. Causal Inference: For Statistics, Social, and Biomedical Sciences an Introduction (Cambridge University Press, 2015). doi:10.1017/CBO9781139025751 
Study 1: How important is it to you that you only share news articles on social media (such as Facebook and Twitter) if they are accurate?"

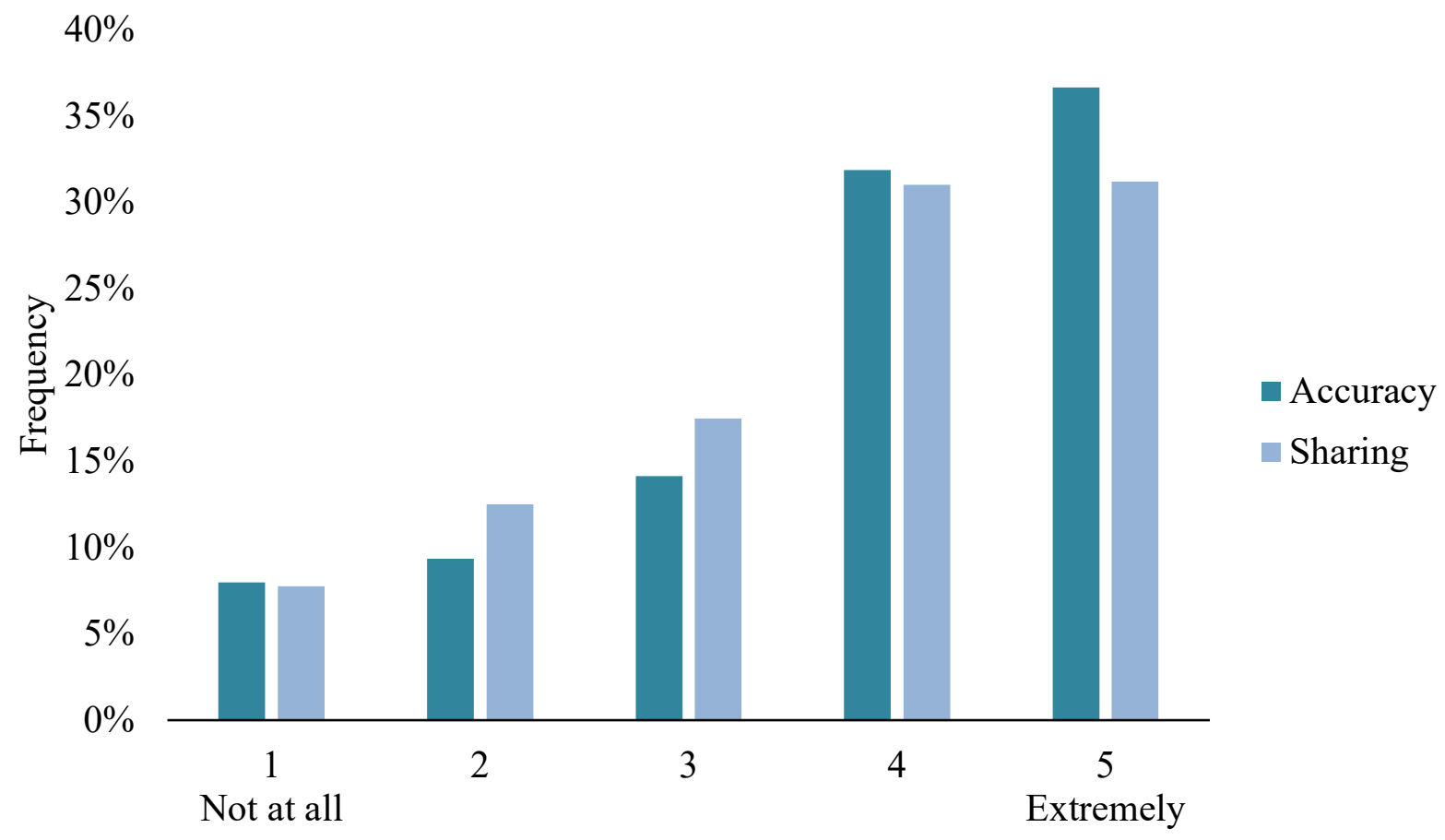

Extended Data Figure 1. Distribution of responses to the post-experimental question "How important is it to you that you only share news articles on social media (such as Facebook and Twitter) if they are accurate" in 1214 Study 1. Responses were directionally lower in the Sharing condition $(\mathrm{M}=3.65, \mathrm{SD}=1.25)$ compared to the Accuracy condition $(\mathrm{M}=3.80, \mathrm{SD}=1.25)$, but the difference was not significant, $\mathrm{t}(1003)=1.83, \mathrm{p}=.067$. 


\begin{tabular}{|l|r|r|r|r|r|r|}
\hline & \multicolumn{3}{|c|}{ Study 4 } & \multicolumn{3}{c|}{ Study 5 } \\
\hline Parameter & Estimate & \multicolumn{2}{|c|}{$\mathbf{9 5 \%}$ CI } & \multicolumn{2}{c|}{ Estimate } & \multicolumn{2}{|c|}{ 95\% CI } \\
\hline$\beta_{P}$ & 0.35 & 0.25 & 0.51 & 1.22 & 0.97 & 1.45 \\
\hline$\beta_{H}$ & -0.12 & -0.21 & 0.12 & 0.57 & 0.40 & 0.87 \\
\hline$p_{1 c}$ & 0.18 & 0.04 & 0.33 & 0.12 & 0.08 & 0.17 \\
\hline$p_{2 c}$ & 0.22 & 0.09 & 0.47 & 0.48 & 0.42 & 0.52 \\
\hline$p_{1 t}$ & 0.51 & 0.30 & 0.57 & 0.18 & 0.14 & 0.22 \\
\hline$p_{2 t}$ & 0.00 & 0.00 & 0.34 & 0.51 & 0.46 & 0.55 \\
\hline$\theta$ & 5.28 & 3.91 & 10.73 & 54.17 & 21.16 & 4091.50 \\
\hline$k$ & -0.12 & -0.20 & -0.05 & -0.03 & -0.18 & 0.05 \\
\hline
\end{tabular}

Overall probability considered in Control:

\begin{tabular}{|l|r|r|r|r|r|r|}
\hline Accuracy & 0.40 & 0.33 & 0.59 & 0.60 & 0.54 & 0.65 \\
\hline Political Concordance & 0.78 & 0.53 & 0.91 & 0.53 & 0.48 & 0.58 \\
\hline Humorousness & 0.82 & 0.67 & 0.96 & 0.88 & 0.83 & 0.92 \\
\hline
\end{tabular}

Overall probability considered in Treatment:

\begin{tabular}{|l|r|r|r|r|r|r|}
\hline Accuracy & 0.51 & 0.46 & 0.64 & 0.68 & 0.63 & 0.73 \\
\hline Political Concordance & 1.00 & 0.66 & 1.00 & 0.49 & 0.45 & 0.54 \\
\hline Humorousness & 0.49 & 0.43 & 0.70 & 0.82 & 0.78 & 0.86 \\
\hline
\end{tabular}

Treatment effect on probability of being considered:

\begin{tabular}{|l|r|r|r|r|r|r|}
\hline Accuracy & 0.11 & 0.03 & 0.20 & 0.09 & 0.01 & 0.16 \\
\hline Political Concordance & 0.22 & 0.07 & 0.35 & -0.03 & -0.10 & 0.03 \\
\hline Humorousness & -0.33 & -0.48 & -0.14 & -0.06 & -0.12 & 0.00 \\
\hline
\end{tabular}

\section{Extended Data Table 1. Best-fit parameter values and quantities of interest for the limited-attention utility} model described in SI Section 3 fit to experimental data from Study 4 and Study 5 . The parameters $\beta_{\mathrm{P}}$ and $\beta_{\mathrm{H}}$ indicate preference for partisan alignment and humorousness, respectively, relative to accuracy; $\mathrm{p}_{1 \mathrm{c}}, \mathrm{p}_{2 \mathrm{c}}, \mathrm{p}_{1 \mathrm{t}}$, and $\mathrm{p}_{2 \mathrm{t}}$ indicate probabilities of attending to various pairs of preference terms in each condition (which are then used to construct the probabilities indicated lower in the table); and $\theta$ and $\mathrm{k}$ parameterize the sigmoid function that translates utility into choice. The key prediction of the preference-based account is that people care substantially less about accuracy than one or more of the other dimensions - that is, that $\beta_{\mathrm{P}}>1$ and/or $\beta_{\mathrm{H}}>1$. In contrast to this prediction, we see that $\beta_{\mathrm{H}}$ is significantly smaller than 1 in both studies (Study $2 \mathrm{~b}, \mathrm{p}<0.001$; Study $2 \mathrm{c}, \mathrm{p}=0.001$ ), such that participants value accuracy more than humorousness; and $\beta_{\mathrm{P}}$ is significantly less than 1 in Study $2 \mathrm{~b}(\mathrm{p}<$ $0.001)$, and not significantly different from 1 is Study $2 \mathrm{c}(\mathrm{p}=0.065)$, such that participants value accuracy as much or more than political concordance. Thus, we find no evidence that participants care more about partisanship than accuracy. Conversely, this observation is consistent with the inattention-based account's prediction that participants value accuracy as much or more than other dimensions. The results also confirm the inattention-based account's second prediction that by default (i.e. in the control), participants will often fail to consider accuracy. Accordingly, we see that the probability of considering accuracy in the control is substantially lower than 1 (Study $2 \mathrm{~b}, 0.40$ $[0.33,0.59]$; Study $2 c, 0.60[0.54,0.65])$. The confirmation of these two predictions provides quantitative support for the claim that inattention to accuracy plays an important role in the share of misinformation in the control condition. Finally, the results confirm the inattention-based account's third prediction, namely that priming accuracy in the treatment will increase attention to accuracy; the probability that participants consider accuracy is significantly higher in the treatment compared to the control (Study 2b, $\mathrm{p}=0.005$; Study 2c, $\mathrm{p}=0.016$ ). 
Study 3

False Headlines

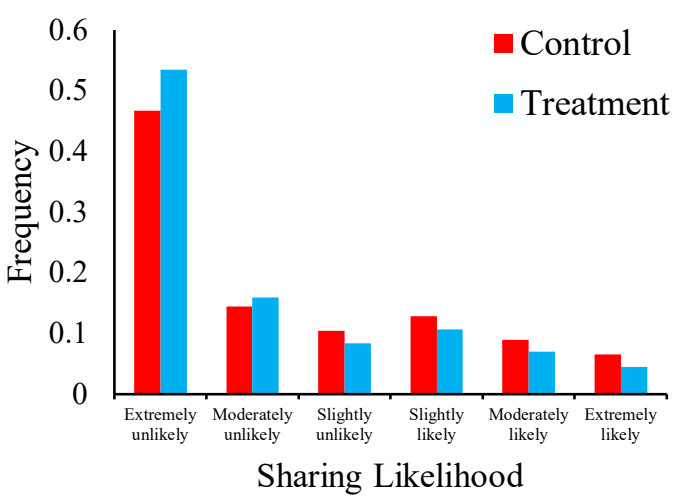

Study 4

False Headlines

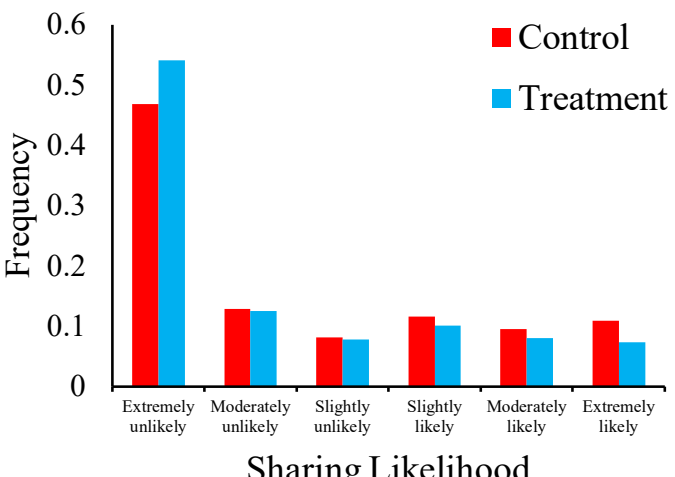

Study 3

True Headlines

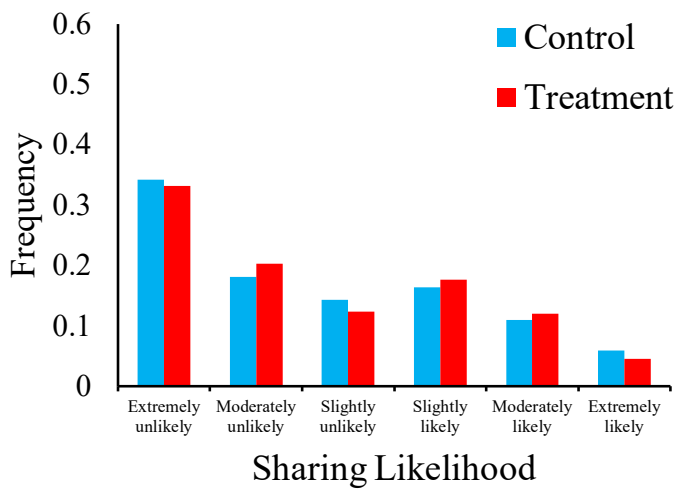

Study 4

True Headlines

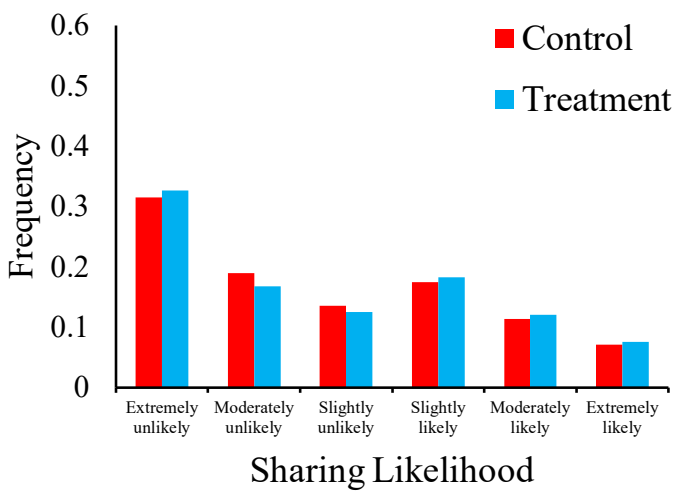

Extended Data Figure 2. Distribution of sharing intentions in Study 3 and Study 4, by condition and headline veracity. Whereas Figure 2 discretizes the sharing intention variable for ease of interpretation such that all "unlikely" responses are scored as 0 and all "likely" responses are scored as 1, here the full distributions are shown. The regression models use these non-discretized values (scored from 1 to 6). 
Study 5

False Headlines

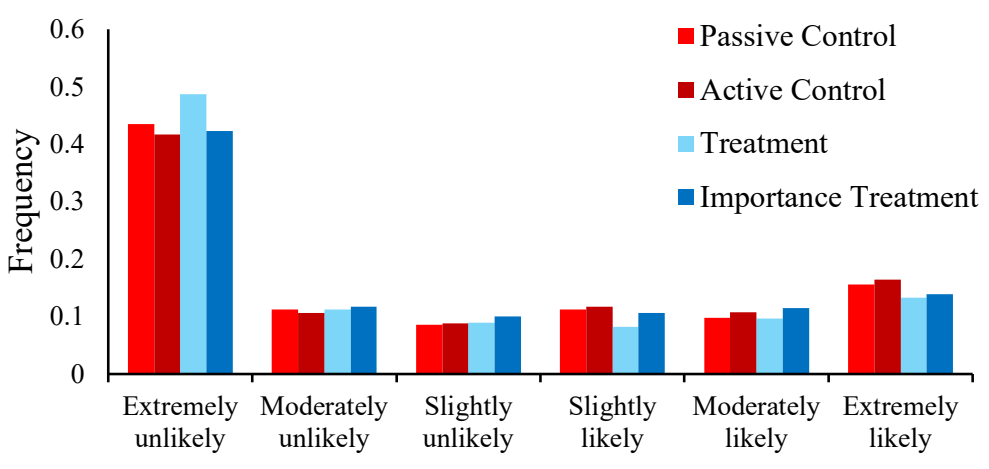

Sharing Likelihood

Study 5

True Headlines

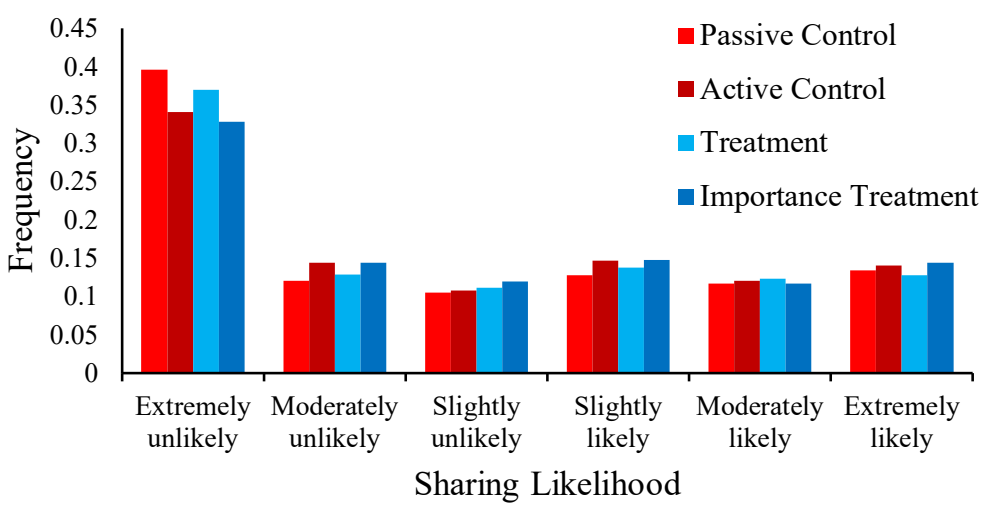

Extended Data Figure 3. Distribution of sharing intentions in Study 5, by condition and headline veracity.

Whereas Figure 2 discretizes the sharing intention variable for ease of interpretation such that all "unlikely" responses are scored as 0 and all "likely" responses are scored as 1 , here the full distributions are shown. The regression models use these non-discretized values (scored from 1 to 6 ). 

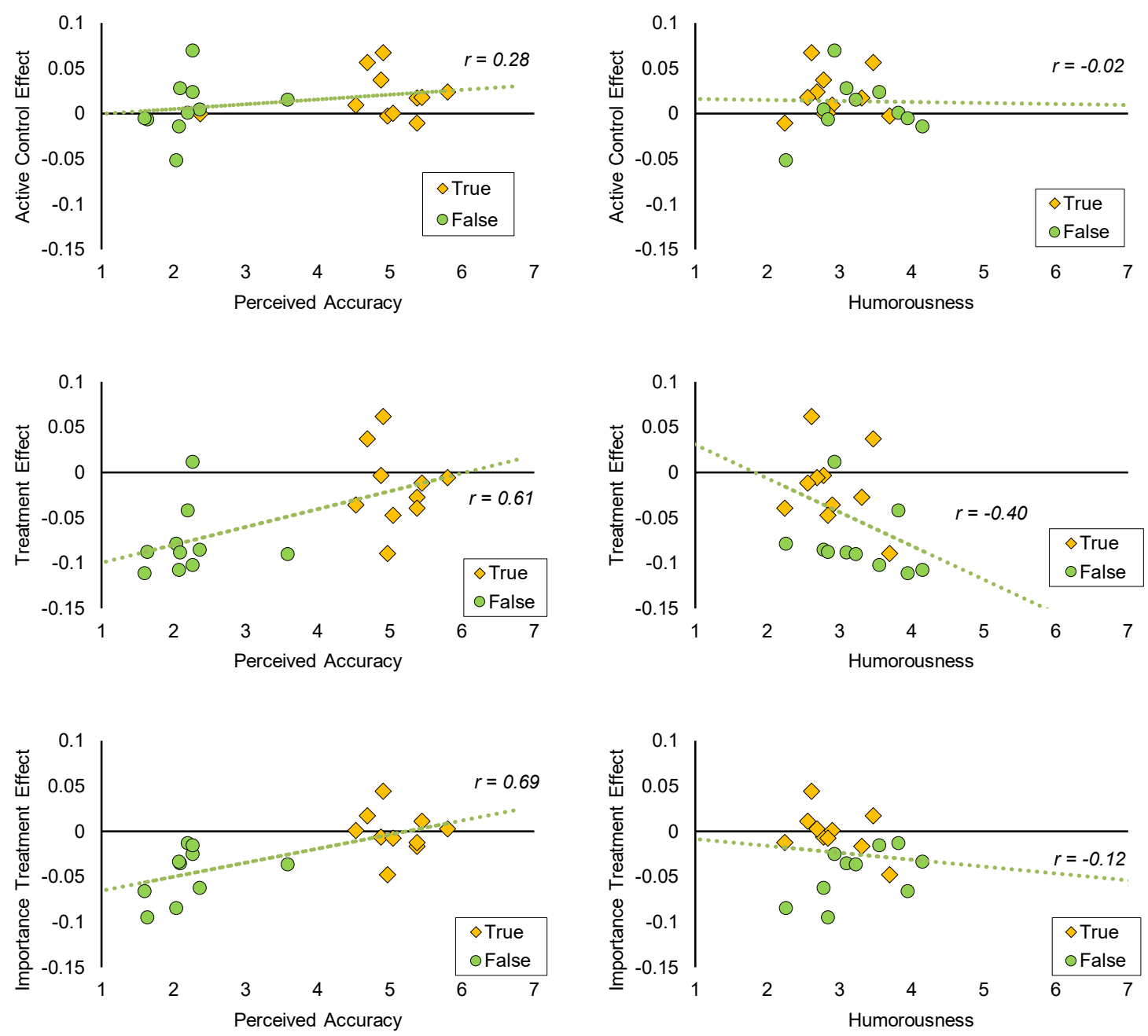

1258
Extended Data Figure 4. Headline-level analyses for Study 5 showing the effect of each condition relative to control as a function of the headlines' perceived accuracy and humorousness. For each headline, we calculate the effect size as the mean sharing intention in the condition in question minus the control (among users who indicate that they sometimes share political content); and we then plot this difference against the headline's pre-test rating of perceived accuracy and humorousness. As can be seen, both the effect of both treatments is strongly correlated with the perceived accuracy of headline (Treatment, $r(18)=0.61, p=0.005$; Importance Treatment, $r(18)=.69, p=0.0008)$, such that both treatments reduce sharing intentions to a greater extent as the headline becomes more inaccurate seeming. This supports our proposed mechanism whereby the treatments operate through drawing attention to the concept of accuracy. Importantly, we see no such analogous effect for the active control: Drawing attention to the concept of humorousness does not make people significantly less likely to share less humorous headlines (or more likely to share more humorous headlines), $r(18)=-0.02, p=.93$. This confirms the prediction generated by our model fitting in SI Section 3.6 - because our participants do not have a preference for sharing humorous news headlines, drawing their attention to humorousness does not influence their choices. This also demonstrates the importance of our theoretical approach that incorporates the role of preferences, relative to how priming is often conceptualized in psychology: drawing attention to a concept does not automatically lead to a greater impact of that concept on behavior. 


\begin{tabular}{|l|c|c|c|c|c|c|}
\hline & \multicolumn{3}{|c|}{ Political content sharers } & \multicolumn{3}{c|}{ All participants } \\
\hline & Aggregate & Round 1 & Round 2 & Aggregate & Round 1 & Round 2 \\
\hline Inattention & $51.2 \%$ & $53.7 \%$ & $50.2 \%$ & $50.8 \%$ & $48.7 \%$ & $51.6 \%$ \\
\hline Confusion & $33.1 \%$ & $28.1 \%$ & $35.0 \%$ & $33.2 \%$ & $31.3 \%$ & $34.1 \%$ \\
\hline Purposeful sharing & $15.8 \%$ & $18.2 \%$ & $14.8 \%$ & $16.0 \%$ & $20.0 \%$ & $14.3 \%$ \\
\hline
\end{tabular}

1278 Extended Data Table 2. Fraction of sharing of false content attributable to inattention, confusion, and 1279 purposeful sharing in Study 6. The results are extremely similar across rounds of data collection, and when 1280

1281

1282

1283

1284

\begin{tabular}{|l|l|l|l|l|l|l|l|l|l|l|l|}
\hline Wave & Date Range & $\begin{array}{l}\text { Treatment } \\
\text { Time }\end{array}$ & $\begin{array}{l}\text { Treatment } \\
\text { Days }\end{array}$ & Bots & $\begin{array}{l}\text { Users } \\
\text { Followed }\end{array}$ & $\begin{array}{l}\text { Follow- } \\
\text { backs }\end{array}$ & $\begin{array}{l}\text { Qualified } \\
\text { Users }\end{array}$ & $\begin{array}{l}\text { DMs } \\
\text { sent }\end{array}$ & $\begin{array}{l}\text { Link } \\
\text { clicks }\end{array}$ & $\begin{array}{l}\text { Rated } \\
\text { tweets } \\
\text { analyzed }\end{array}$ & $\begin{array}{l}\text { Total tweets } \\
\text { analyzed }\end{array}$ \\
\hline 1 & $\begin{array}{l}4 / 20 / 2018- \\
4 / 27 / 2018\end{array}$ & $\begin{array}{l}7: 43 \mathrm{pm} \\
\text { EST }\end{array}$ & $\begin{array}{l}7 \\
\text { (no 4/25) }\end{array}$ & 6 & 19,913 & 821 & 705 & 705 & 80 & 12,912 & 231,162 \\
\hline 2 & $\begin{array}{l}9 / 12 / 2018- \\
9 / 14 / 2018\end{array}$ & $\begin{array}{l}5: 00 \mathrm{pm} \\
\text { EST }\end{array}$ & 3 & 7 & 23,673 & 3,111 & 2,153 & 1,060 & 60 & 24,912 & 387,993 \\
\hline 3 & $\begin{array}{l}1 / 28 / 2019- \\
2 / 08 / 2019\end{array}$ & $\begin{array}{l}7: 00 \mathrm{pm} \\
\text { EST }\end{array}$ & 12 & 13 & 92,793 & 7,432 & 2,521 & 2,330 & 169 & 15,918 & 564,843 \\
\hline Total & & & 23 & 13 & 136,379 & 11,364 & 5,379 & 4,095 & 309 & 53,742 & $1,183,998$ \\
\hline
\end{tabular}

Extended Data Table 3. Details for the three waves of Study 7 data collection.
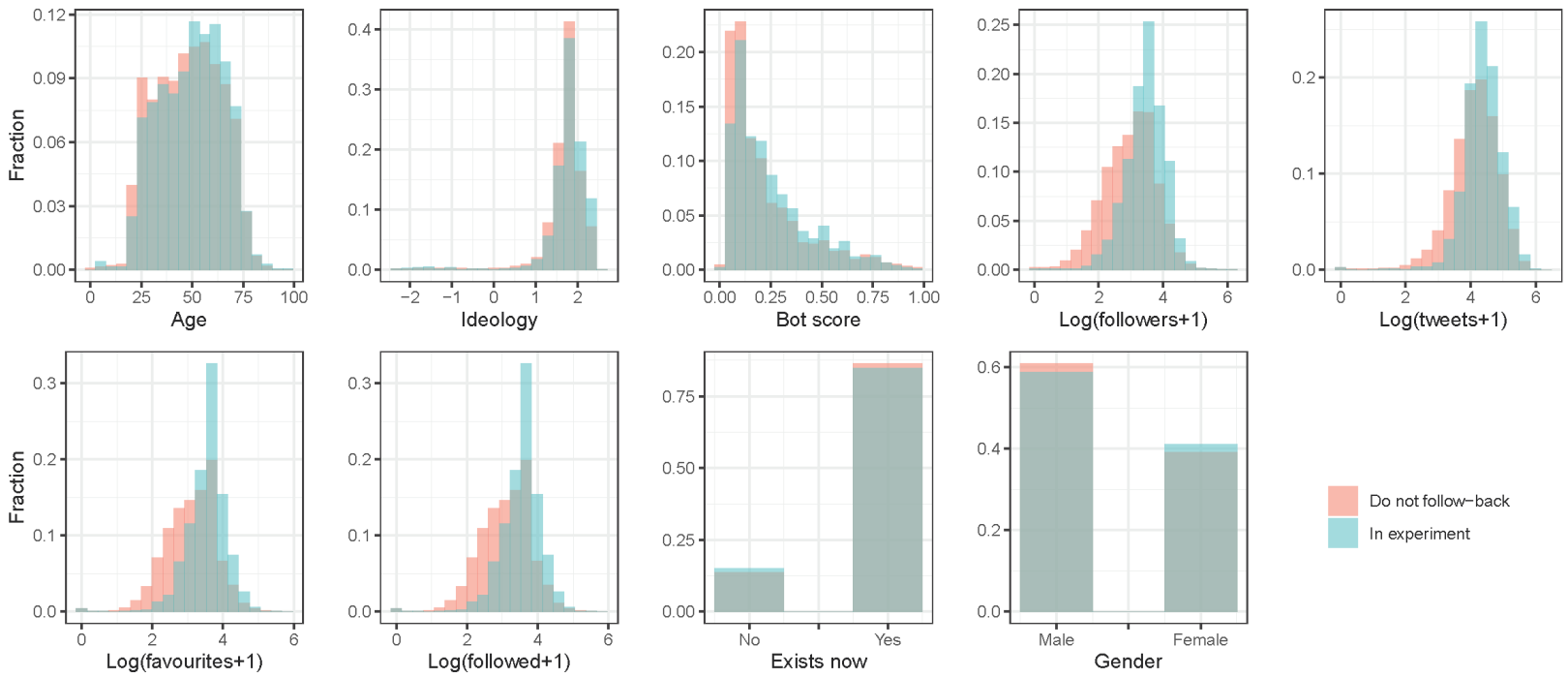

Do not follow-back

In experiment

Extended Data Figure 5. Characteristics of the users in the Study 7 Twitter field experiment (blue) compared to a 


\begin{tabular}{|c|c|c|c|c|c|c|c|c|c|c|c|c|}
\hline \multirow{2}{*}{$\begin{array}{l}\text { Tweet } \\
\text { Type }\end{array}$} & \multirow{2}{*}{$\begin{array}{l}\text { Article } \\
\text { Type }\end{array}$} & \multirow{2}{*}{$\begin{array}{c}\text { Randomization- } \\
\text { Failure }\end{array}$} & \multirow{2}{*}{$\begin{array}{l}\text { Model } \\
\text { Spec }\end{array}$} & \multicolumn{3}{|c|}{ Average Relative Quality } & \multicolumn{3}{|c|}{ Summed Relative Quality } & \multicolumn{3}{|c|}{ Discernment } \\
\hline & & & & Coeff & $\operatorname{Reg} \mathbf{p}$ & FRI p & Coeff & $\operatorname{Reg} \mathbf{p}$ & FRI p & Coeff & $\operatorname{Reg} \mathbf{p}$ & FRI p \\
\hline All & All & ITT & Wave FE & 0.007 & 0.004 & 0.009 & 0.011 & 0.022 & 0.117 & 0.061 & 0.004 & 0.016 \\
\hline All & All & ITT & Wave PS & 0.007 & 0.006 & 0.009 & 0.010 & 0.020 & 0.098 & 0.059 & 0.004 & 0.018 \\
\hline All & All & ITT & Date FE & 0.006 & 0.019 & 0.040 & 0.009 & 0.070 & 0.267 & 0.053 & 0.019 & 0.055 \\
\hline All & All & ITT & Date PS & 0.006 & 0.041 & 0.035 & 0.008 & 0.087 & 0.179 & 0.050 & 0.028 & 0.052 \\
\hline All & All & Exclude & Wave FE & 0.007 & 0.008 & 0.027 & 0.013 & 0.007 & 0.074 & 0.065 & 0.003 & 0.016 \\
\hline All & All & Exclude & Wave PS & 0.007 & 0.011 & 0.024 & 0.012 & 0.009 & 0.068 & 0.062 & 0.003 & 0.019 \\
\hline All & All & Exclude & Date FE & 0.005 & 0.045 & 0.102 & 0.010 & 0.044 & 0.213 & 0.053 & 0.020 & 0.062 \\
\hline All & All & Exclude & Date PS & 0.005 & 0.069 & 0.067 & 0.009 & 0.071 & 0.159 & 0.051 & 0.032 & 0.062 \\
\hline RT & All & ITT & Wave FE & 0.007 & 0.003 & 0.004 & 0.012 & 0.007 & 0.029 & 0.058 & 0.001 & 0.003 \\
\hline RT & All & ITT & Wave PS & 0.007 & 0.004 & 0.004 & 0.011 & 0.006 & 0.020 & 0.055 & 0.001 & 0.003 \\
\hline $\mathrm{RT}$ & All & ITT & Date FE & 0.006 & 0.017 & 0.014 & 0.010 & 0.032 & 0.060 & 0.050 & 0.008 & 0.006 \\
\hline $\mathrm{RT}$ & All & ITT & Date PS & 0.006 & 0.027 & 0.012 & 0.009 & 0.042 & 0.035 & 0.047 & 0.016 & 0.013 \\
\hline RT & All & Exclude & Wave FE & 0.007 & 0.004 & 0.009 & 0.014 & 0.002 & 0.011 & 0.059 & 0.001 & 0.003 \\
\hline RT & All & Exclude & Wave PS & 0.007 & 0.005 & 0.008 & 0.013 & 0.002 & 0.011 & 0.057 & 0.001 & 0.004 \\
\hline RT & All & Exclude & Date FE & 0.006 & 0.032 & 0.032 & 0.011 & 0.018 & 0.038 & 0.049 & 0.010 & 0.008 \\
\hline RT & All & Exclude & Date PS & 0.006 & 0.042 & 0.023 & 0.010 & 0.033 & 0.027 & 0.047 & 0.021 & 0.017 \\
\hline All & No Opinion & ITT & Wave FE & 0.007 & 0.002 & 0.015 & 0.012 & 0.012 & 0.115 & 0.061 & 0.004 & 0.017 \\
\hline All & No Opinion & ITT & Wave PS & 0.007 & 0.004 & 0.016 & 0.011 & 0.011 & 0.100 & 0.058 & 0.004 & 0.021 \\
\hline All & No Opinion & ITT & Date FE & 0.006 & 0.015 & 0.057 & 0.010 & 0.051 & 0.271 & 0.054 & 0.016 & 0.047 \\
\hline All & No Opinion & ITT & Date PS & 0.006 & 0.031 & 0.044 & 0.009 & 0.063 & 0.179 & 0.054 & 0.018 & 0.034 \\
\hline All & No Opinion & Exclude & Wave FE & 0.007 & 0.005 & 0.037 & 0.014 & 0.003 & 0.067 & 0.064 & 0.003 & 0.015 \\
\hline All & No Opinion & Exclude & Wave PS & 0.007 & 0.008 & 0.035 & 0.013 & 0.005 & 0.066 & 0.060 & 0.003 & 0.019 \\
\hline All & No Opinion & Exclude & Date FE & 0.006 & 0.033 & 0.130 & 0.011 & 0.027 & 0.205 & 0.056 & 0.015 & 0.047 \\
\hline All & No Opinion & Exclude & Date PS & 0.006 & 0.051 & 0.080 & 0.010 & 0.047 & 0.149 & 0.055 & 0.019 & 0.036 \\
\hline RT & No Opinion & ITT & Wave FE & 0.008 & 0.001 & 0.003 & 0.012 & 0.003 & 0.023 & 0.057 & 0.001 & 0.004 \\
\hline RT & No Opinion & ITT & Wave PS & 0.008 & 0.002 & 0.004 & 0.012 & 0.003 & 0.019 & 0.054 & 0.001 & 0.004 \\
\hline RT & No Opinion & ITT & Date FE & 0.007 & 0.009 & 0.013 & 0.010 & 0.022 & 0.059 & 0.051 & 0.006 & 0.007 \\
\hline RT & No Opinion & ITT & Date PS & 0.007 & 0.013 & 0.007 & 0.010 & 0.026 & 0.028 & 0.050 & 0.010 & 0.008 \\
\hline $\mathrm{RT}$ & No Opinion & Exclude & Wave FE & 0.008 & 0.001 & 0.009 & 0.014 & 0.001 & 0.008 & 0.058 & 0.001 & 0.004 \\
\hline RT & No Opinion & Exclude & Wave PS & 0.008 & 0.003 & 0.008 & 0.013 & 0.001 & 0.009 & 0.056 & 0.001 & 0.005 \\
\hline RT & No Opinion & Exclude & Date FE & 0.006 & 0.017 & 0.029 & 0.011 & 0.010 & 0.030 & 0.051 & 0.007 & 0.008 \\
\hline RT & No Opinion & Exclude & Date PS & 0.006 & 0.021 & 0.014 & 0.011 & 0.018 & 0.019 & 0.050 & 0.013 & 0.011 \\
\hline
\end{tabular}

Extended Data Table 4. Coefficients and p-values associated with each model of quality for Study 7. In the model specification column, FE represents fixed effects (i.e. just dummies) and PS represents post-stratification (i.e. centered dummies interacted with the post-treatment dummy). For Discernment, the p-value associated with the interaction between the post-treatment dummy and the source type dummy is reported; for all other DVs, the p-value associated with the post-treatment dummy is reported. P-values below 0.05 are bolded. Taken together, the results support the conclusion that the treatment significantly increased the quality of news shared. For the average relative quality score, virtually all (57 out of 64) analyses found a significant effect. For the summed relative quality score, most analyses found a significant effect, except for the FRI-derived p-values when including all tweets which were all nonsignificant. For discernment, 60 out of 64 analyses found a significant effect. Reassuringly, there was little qualitative difference between the two approaches for handling randomization failure, or across the four model specifications; and $98 \%$ of results were significant when only considering retweets without comment (which are the low-engagement sharing decisions that our theory predicts should respond to the treatment). 

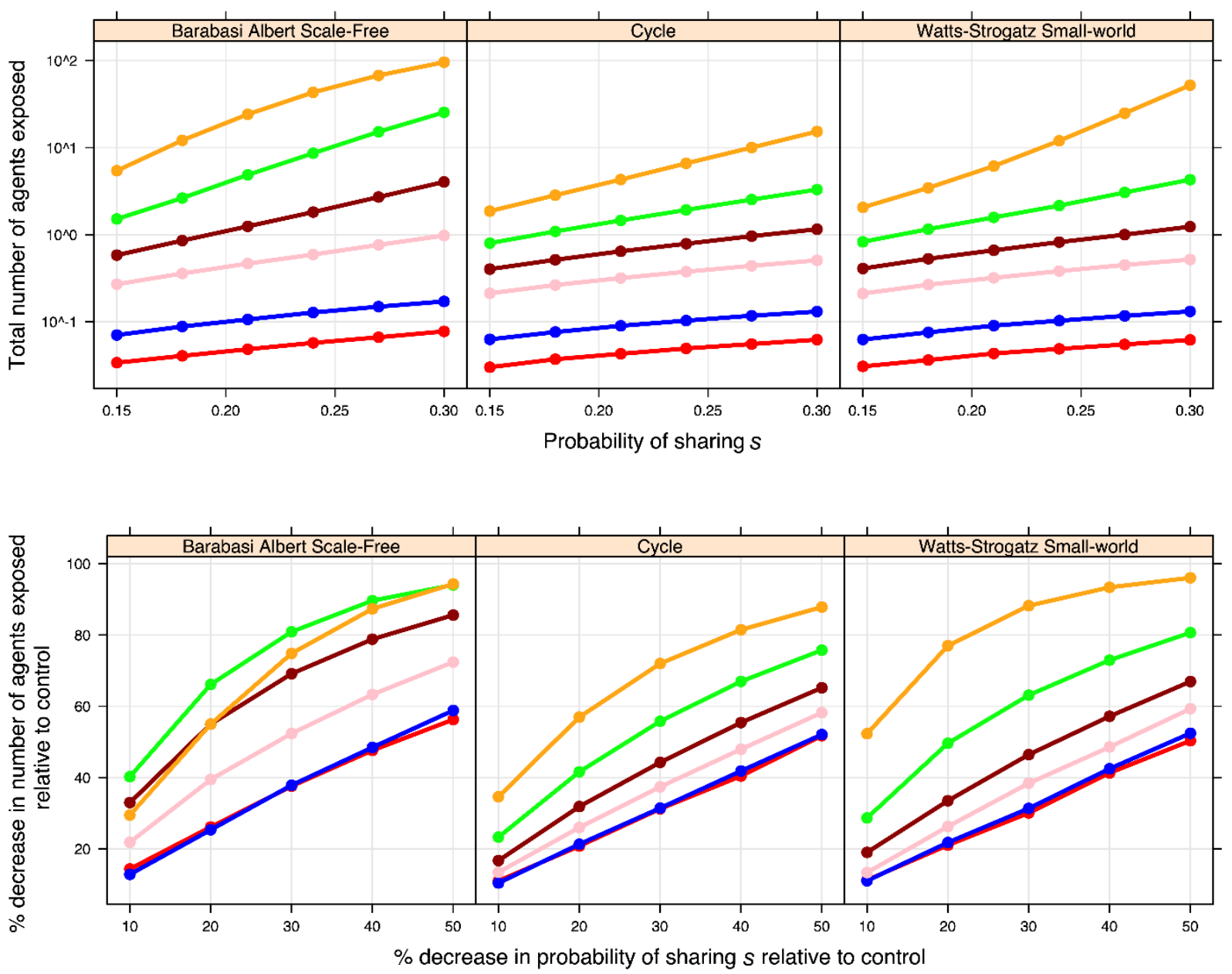

Fraction of population each agent is connected to $(\mathrm{k} / \mathrm{N})$

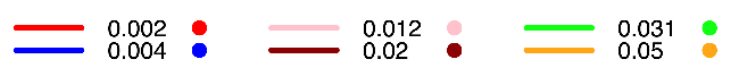

Extended Data Figure 6. Results of agent-based simulations of news sharing on social networks from SI Section 6. Shown is the relationship between individual-level probability of sharing misinformation and populationlevel exposure rates, for various levels of network density (fraction of the population that the average agent is connected to, $k / N$ ). Top row shows the raw number of agents exposed to the misinformation (y-axis) as a function of the agents' raw probability of misinformation sharing (x-axis). Bottom row shows the percent reduction in the fraction of the population exposed to the piece of misinformation relative to control (y-axis) as a function of the percent reduction in individuals' probability of sharing the misinformation relative to control (x-axis). As can be seen, a robust pattern emerges across network structures. First, we see that the network dynamics never suppress the individual-level intervention effect: a decrease in sharing probability of $\mathrm{X} \%$ always decreases the fraction of the population exposed to the misinformation by at least $\mathrm{X} \%$. Second, in some cases the network dynamics can dramatically amplify the impact of the individual-level intervention: for example, a $10 \%$ decrease in sharing probability can lead to up to a $40 \%$ decrease in the fraction of the population that is exposed, and a $50 \%$ decrease in sharing probability can lead to over a $95 \%$ reduction in the fraction of the population that is exposed. These simulation results help to connect our findings about individual-level sharing to the resulting impacts on populationlevel spreading dynamics of misinformation. They demonstrate the potential for individual-level interventions, such as the accuracy nudges that we propose here, to meaningfully improve the quality of the information that is spread 
1326 via social media. These simulations also lay the groundwork for future theoretical work that can investigate a range

1327 of issues, including which agents to target if only a limited number of agents can be intervened on, the optimal

1328 spatiotemporal intervention schedule to minimize the frequency of any individual agent receiving the intervention

1329 (to minimize adaption/familiarity effects), and the inclusion of strategic sharing considerations (by introducing game 1330 theory). 
Acknowledgments The authors gratefully acknowledge helpful feedback and comments from Adam Bear, Jillian Jordan, David Lazer, and Tage Rai, Bjarke Mønsted, as well as funding from the Ethics and Governance of Artificial Intelligence Initiative of the Miami Foundation, the William and Flora Hewlett Foundation, the Omidyar Network, the John Templeton Foundation grant \#61061, the Canadian Institutes of Health Research, and the Social Sciences and Humanities Research Council of Canada.

\section{Author Contribution Statement}

GP and DR conceived of the research; GP and DR designed the survey experiments; AA and GP conducted the survey experiments; GP and DR analyzed the survey experiments; ZE, MM, and DR designed the Twitter experiment; ZE, MM, and AA conducted the Twitter experiment; ZE, MM, DE, and DR analyzed the Twitter experiment; DR designed and analyzed the limit-attention utility model; MM and DR designed and analyzed the network simulations; GP and DR wrote the paper, with input from ZE, MM, AA, and DE. All authors approved the final manuscript.

\section{Competing Interest}

Other research by D.E. is funded by Facebook, which has also sponsored a conference he co-organizes. D.E. previously had a significant financial interest in Facebook while contributing to this research. Other research by D.R. is funded by a gift from Google.

\section{Additional Information}

Correspondence and requests for materials should be addressed to Gordon Pennycook or David Rand.

\section{Data Availability Statement}

Data and materials for Studies 1 through 6 are available at https://osf.io/p6u8k/. Due to privacy concerns, data from Study 7 are available upon request.

\section{Code Availability Statement}

Code for all studies is available at https://osf.io/p6u $8 \mathrm{k} /$. 


\title{
Supplementary Materials
}

\author{
for \\ Simple accuracy nudges can reduce misinformation online
}

\section{Pre-tests}

\section{Study 1}

The pretest asked participants ( $N=2,008$ from MTurk, $N=1,988$ from Lucid) to rate 10 randomly selected news headlines (from a corpus of 70 false, or 70 misleading/hyperpartisan, or 70 true) on a number of dimensions. Of primary interest, participants were asked the following question: "Assuming the above headline is entirely accurate, how favorable would it be to Democrats versus Republicans" $-1=$ More favorable for Democrats, $5=$ More favorable for Republicans). We used data from this question to selected the items used in Study 1 such that the Pro-Democratic items were equally different from the scale midpoint as the Pro-Republican items within the true and false categories. Participants were also asked to rate the headlines on the following dimensions: Plausibility ("What is the likelihood that the above headline is true" $1=$ Extremely unlikely, 7 = Extremely likely), Importance ("Assuming the headline is entirely accurate, how important would this news be?" - $1=$ Extremely unimportant, $5=$ Extremely important), Excitingness ("How exciting is this headline" - $1=$ not at all, $5=$ extremely), Worryingness ("How worrying is this headline?" - $1=$ not at all, $5=$ extremely), and Familiarity ("Are you familiar with the above headline (have you seen or heard about it before)?" Yes/Unsure/No). Participants were also asked to indicate whether they would be willing to share each presented headline ("If you were to see the above article on social media, how likely would you be to share it?" - 1 = Extremely unlikely, 7 = Extremely likely). The pretest was run on June $24^{\text {th }}, 2019$.

\section{Studies 3 and 6}

For the pretest (completed on June $\left.1^{\text {st }}, 2017\right)$, participants $(N=209$ from MTurk) rated 25 false headlines or 25 true headlines on the following dimensions: Plausibility ("What is the likelihood that the above headline is true" - 1 = Extremely unlikely, 7 = Extremely likely), Partisanship ("Assuming the above headline is entirely accurate, how favorable would it be to Democrats versus Republicans" - 1 = More favorable for Democrats, 5 = More favorable for Republicans), and Familiarity ("Are you familiar with the above headline (have you seen or heard about it before)?” -Yes/ Unsure/ No).

\section{Study 4}

For the pretest (completed on November 22 $\left.{ }^{\text {nd }}, 2017\right)$, participants ( $N=269$ from MTurk) rated 36 false headlines or 36 true headlines on the following dimensions: Plausibility ("What is the likelihood that the above headline is true" - 1 = Extremely unlikely, 7 = Extremely likely), Partisanship ("Assuming the above headline is entirely accurate, how favorable would it be to Democrats versus Republicans" - 1 = More favorable for Democrats, $5=$ More favorable for Republicans), Familiarity ("Are you familiar with the above headline (have you seen or heard about it before)?" -Yes/Unsure/No), and Humorousness ("In your opinion, is the above headline funny, amusing, or entertaining" 1 = extremely unfunny, 7 = extremely funny). 


\section{Study 5}

The pretest asked participants ( $N=516$ from MTurk) to rate a random subset of 30 headlines from a larger set of false, hyperpartisan, and true headlines (there were 40 headlines in total in each category) on the following dimensions: Plausibility ("What is the likelihood that the above headline is true" - 1 = Extremely unlikely, 7 = Extremely likely), Partisanship ("Assuming the above headline is entirely accurate, how favorable would it be to Democrats versus Republicans" - 1 = More favorable for Democrats, 5 = More favorable for Republicans), Familiarity ("Are you familiar with the above headline (have you seen or heard about it before)?" - Yes/Unsure/No), Funniness ("In your opinion, is the above headline funny, amusing, or entertaining" $1=$ extremely unfunny, 7 = extremely funny). The pretest was completed on May $23^{\text {rd }}, 2018$. 


\section{Regression tables}

The full regression models are shown for Study 1 analyses in Table S1, for Studies 3 and 4 in Tables S2 and S3, and for Study 5 in Tables S4 and S5. 


\begin{tabular}{|c|c|c|c|}
\hline & $\begin{array}{c}(1) \\
\text { Linear } \\
\text { Rating }\end{array}$ & $\begin{array}{c}(2) \\
\text { Logistic } \\
\text { Rating }\end{array}$ & $\begin{array}{c}(3) \\
\text { Linear } \\
z \text {-Rating }\end{array}$ \\
\hline Condition $($ Accuracy $=-0.5$, Sharing $=0.5$ ) & $\begin{array}{c}-0.109 * * * \\
(0.0181) \\
1.65 \mathrm{e}-09\end{array}$ & $\begin{array}{c}-0.381 * * * \\
(0.102) \\
0.000186\end{array}$ & $\begin{array}{c}-0.000407 \\
(0.0377) \\
0.991\end{array}$ \\
\hline Veracity $($ False $=-0.5$, True $=0.5)$ & $\begin{array}{c}0.309 * * * \\
(0.0204) \\
<1 \mathrm{e}-10\end{array}$ & $\begin{array}{c}1.460 * * * \\
(0.109) \\
<1 \mathrm{e}-10\end{array}$ & $\begin{array}{c}0.627 * * * * \\
(0.0422) \\
<1 \mathrm{e}-10\end{array}$ \\
\hline $\begin{array}{l}\text { Concordance of headline }(-0.5=\text { discordant, } \\
0.5=\text { concordant })\end{array}$ & $0.147^{* * *}$ & $0.741^{* * *}$ & $0.308 * * *$ \\
\hline & $\begin{array}{c}(0.0180) \\
<1 \mathrm{e}-10\end{array}$ & $\begin{array}{c}(0.0992) \\
<1 \mathrm{e}-10\end{array}$ & $\begin{array}{c}(0.0376) \\
<1 \mathrm{e}-10\end{array}$ \\
\hline Condition X Veracity & $\begin{array}{c}-0.500 * * * \\
(0.0310) \\
<1 \mathrm{e}-10\end{array}$ & $\begin{array}{c}-2.394 * * * \\
(0.181) \\
<1 \mathrm{e}-10\end{array}$ & $\begin{array}{c}-1.001 * * * \\
(0.0637) \\
<1 \mathrm{e}-10\end{array}$ \\
\hline Condition X Concordance & $\begin{array}{c}0.0917 * * * \\
(0.0221) \\
3.31 \mathrm{e}-05\end{array}$ & $\begin{array}{c}0.317 * * \\
(0.115) \\
0.00569\end{array}$ & $\begin{array}{c}0.208 * * * \\
(0.0462) \\
6.97 \mathrm{e}-06\end{array}$ \\
\hline Veracity X Concordance & $\begin{array}{c}0.0766^{*} \\
(0.0348) \\
0.0274\end{array}$ & $\begin{array}{c}0.252 \\
(0.191) \\
0.188\end{array}$ & $\begin{array}{c}0.159 * \\
(0.0723)\end{array}$ \\
\hline Condition X Veracity X Concordance & $\begin{array}{c}-0.0207 \\
(0.0396) \\
0.601\end{array}$ & $\begin{array}{c}-0.0394 \\
(0.203) \\
0.846\end{array}$ & $\begin{array}{c}-0.0340 \\
(0.0827) \\
0.681\end{array}$ \\
\hline Constant & $\begin{array}{c}0.379 * * * \\
(0.0113) \\
<1 \mathrm{e}-10\end{array}$ & $\begin{array}{c}-0.583 * * * \\
(0.0596) \\
<1 \mathrm{e}-10\end{array}$ & $\begin{array}{c}0.000203 \\
(0.0234) \\
0.993\end{array}$ \\
\hline Observations & 36,180 & 36,180 & 36,180 \\
\hline Participant clusters & 1005 & 1005 & 1005 \\
\hline Headline clusters & 36 & 36 & 36 \\
\hline R-squared & 0.207 & & 0.189 \\
\hline
\end{tabular}

Table S1. Regressions with robust standard errors clustered on participant and headline predicting responses $(0$ or 1) in Study 1. Models 1 and 3 use linear regression; Model 2 uses logistic regression. Models 1 and 2 use the raw responses; Model 3 uses responses that are z-scored within condition. We observe a significant main effect of condition in Models 1 and 2, such that overall, participants were more likely to rate headlines as true than to say they would consider sharing them (this difference is eliminated by design in Model 3 because responses are z-scored within condition). Across all 3 models, we unsurprisingly observe significant positive main effects of veracity and concordance ( $p<.001$ for both main effects in all models). Critically, as predicted, across all models we observe a significant negative interaction between condition and veracity, and a significant positive interaction between condition and headline concordance ( $p<.001$ for both interactions in all models). Thus, participants are less sensitive to veracity, and more sensitive to concordance, when making sharing decisions than accuracy judgments. We also observe no significant 3-way interaction ( $p>.100$ in all models). Finally, we see inconsistent evidence regarding a positive interaction between veracity and concordance, such that veracity may or may not play a bigger role among concordant headlines than discordant headlines. 


\begin{tabular}{|c|c|c|c|c|c|c|}
\hline & \multicolumn{3}{|c|}{$\begin{array}{l}(1) \quad(2) \\
\text { Participants that share political content }\end{array}$} & \multicolumn{3}{|c|}{$\begin{array}{c}\text { (5) } \\
\text { All participants }\end{array}$} \\
\hline & S3 & S4 & $\mathrm{S} 3+\mathrm{S} 4$ & S3 & S4 & $\mathrm{S} 3+\mathrm{S} 4$ \\
\hline Treatment & $\begin{array}{c}-0.0545 * * * \\
(0.0145) \\
0.000176\end{array}$ & $\begin{array}{c}-0.0582 * * * \\
(0.0168) \\
0.000536\end{array}$ & $\begin{array}{c}-0.0557 * * * \\
(0.0110) \\
3.71 \mathrm{e}-07\end{array}$ & $\begin{array}{c}-0.0294 * \\
(0.0117) \\
0.0117\end{array}$ & $\begin{array}{c}-0.0457 * * * \\
(0.0139) \\
0.000977\end{array}$ & $\begin{array}{c}-0.0372 * * * \\
(0.00902) \\
3.79 \mathrm{e}-05\end{array}$ \\
\hline Veracity $(0=$ False, $1=$ True $)$ & $\begin{array}{c}0.0540 * * \\
(0.0205) \\
0.00832\end{array}$ & $\begin{array}{c}0.0455 \\
(0.0271) \\
0.0934\end{array}$ & $\begin{array}{c}0.0494 * * \\
(0.0161) \\
0.00212\end{array}$ & $\begin{array}{c}0.0383 * \\
(0.0169) \\
0.0237\end{array}$ & $\begin{array}{c}0.0378 \\
(0.0225) \\
0.0935\end{array}$ & $\begin{array}{c}0.0380 * * \\
(0.0138) \\
0.00590\end{array}$ \\
\hline Treatment X Veracity & $\begin{array}{c}0.0529 * * * \\
(0.0108) \\
8.74 \mathrm{e}-07\end{array}$ & $\begin{array}{c}0.0648^{* * *} \\
(0.0147) \\
9.97 \mathrm{e}-06\end{array}$ & $\begin{array}{c}0.0589 * * * \\
(0.00857) \\
<1 \mathrm{e}-10\end{array}$ & $\begin{array}{c}0.0475 * * * \\
(0.00818) \\
6.69 \mathrm{e}-09\end{array}$ & $\begin{array}{c}0.0635^{* * *} \\
(0.0117) \\
5.12 \mathrm{e}-08\end{array}$ & $\begin{array}{c}0.0557 * * * \\
(0.00681) \\
<1 \mathrm{e}-10\end{array}$ \\
\hline $\begin{array}{l}z \text {-Party (Prefer Republicans to } \\
\text { Democrats) }\end{array}$ & & & $\begin{array}{c}0.0169 \\
(0.00939) \\
0.0722\end{array}$ & & & $\begin{array}{c}0.00902 \\
(0.00804) \\
0.262\end{array}$ \\
\hline Veracity X Party & & & $\begin{array}{c}0.00322 \\
(0.00930) \\
0.729\end{array}$ & & & $\begin{array}{c}0.00249 \\
(0.00792) \\
0.753\end{array}$ \\
\hline Treatment X Party & & & $\begin{array}{c}0.00508 \\
(0.0106) \\
0.632\end{array}$ & & & $\begin{array}{c}0.0111 \\
(0.00809) \\
0.170\end{array}$ \\
\hline Treatment X Veracity X Party & & & $\begin{array}{c}-0.0159 \\
(0.00864) \\
0.0663\end{array}$ & & & $\begin{array}{c}-0.0113 * \\
(0.00573) \\
0.0495\end{array}$ \\
\hline$z$-Concordance of Headline & & & $\begin{array}{c}0.0684 * * * \\
(0.00723) \\
<1 \mathrm{e}-10\end{array}$ & & & $\begin{array}{c}0.0524 * * * \\
(0.00625) \\
<1 \mathrm{e}-10\end{array}$ \\
\hline Veracity X Concordance & & & $\begin{array}{c}0.00351 \\
(0.0107) \\
0.743\end{array}$ & & & $\begin{array}{c}0.00396 \\
(0.00897) \\
0.659\end{array}$ \\
\hline Treatment X Concordance & & & $\begin{array}{c}-0.0156 * * * \\
(0.00462) \\
0.000760\end{array}$ & & & $\begin{array}{c}-0.00723 * \\
(0.00315) \\
0.0219\end{array}$ \\
\hline Treatment X Veracity X Concordance & & & $\begin{array}{c}0.0224 * * * \\
(0.00527) \\
2.12 \mathrm{e}-05\end{array}$ & & & $\begin{array}{c}0.0163 * * * \\
(0.00290) \\
1.90 \mathrm{e}-08\end{array}$ \\
\hline Party X Concordance & & & $\begin{array}{c}-0.00352 \\
(0.00928) \\
0.704\end{array}$ & & & $\begin{array}{c}-0.00547 \\
(0.00834) \\
0.511\end{array}$ \\
\hline Treatment X Party X Concordance & & & $\begin{array}{c}0.00725 \\
(0.00471)\end{array}$ & & & $\begin{array}{l}0.00930^{*} \\
(0.00440)\end{array}$ \\
\hline
\end{tabular}




\begin{tabular}{|c|c|c|c|c|c|c|}
\hline \multirow[b]{2}{*}{ Veracity X Party X Concordance } & & & 0.124 & & & 0.0347 \\
\hline & & & $\begin{array}{c}0.0157 \\
(0.0135)\end{array}$ & & & $\begin{array}{c}0.0159 \\
(0.0123)\end{array}$ \\
\hline & & & 0.244 & & & 0.194 \\
\hline \multicolumn{7}{|l|}{ Treatment X Veracity X Party X } \\
\hline \multirow[t]{3}{*}{ Concordance } & & & $-0.0136^{* *}$ & & & $-0.0132 * * *$ \\
\hline & & & $(0.00448)$ & & & $(0.00382)$ \\
\hline & & & 0.00241 & & & 0.000562 \\
\hline \multirow[t]{3}{*}{ Constant } & $0.285 * * *$ & $0.314 * * *$ & $0.300 * * *$ & $0.234 * * *$ & $0.263 * * *$ & $0.249 * * *$ \\
\hline & $(0.0152)$ & $(0.0221)$ & $(0.0125)$ & $(0.0128)$ & $(0.0182)$ & $(0.0106)$ \\
\hline & $<1 \mathrm{e}-10$ & $<1 \mathrm{e}-10$ & $<1 \mathrm{e}-10$ & $<1 \mathrm{e}-10$ & $<1 \mathrm{e}-10$ & $<1 \mathrm{e}-10$ \\
\hline \multirow[t]{3}{*}{ Observations } & 17,417 & 18,677 & 36,094 & 27,732 & 29,885 & 57,617 \\
\hline & 727 & 780 & 1,507 & 1,158 & 1,248 & 2,406 \\
\hline & 24 & 24 & 48 & 24 & 24 & 48 \\
\hline R-squared & 0.019 & 0.016 & 0.063 & 0.012 & 0.014 & 0.045 \\
\hline
\end{tabular}

Table S2. Linear regressions predicting sharing intentions (1-6 Likert scale rescaled to [0,1]) in Studies 3 and 4. Robust standard errors clustered on participant and headline. In all cases, we observe (i) the predicted significant positive interaction between treatment and news veracity, such that sharing discernment was higher in the Treatment compared to the Control; (ii) a negative simple effect of condition for false headlines, such that participants were less likely to consider sharing false headlines in the Treatment compared to the Control; and (iii) no significant simple effect of condition for true headlines, such that participants were no less likely to consider sharing true headlines in the Treatment compared to the Control. Turning to potential moderation effects, we examine the regression models in columns 3 and 6 . We see that the Treatment has a significantly larger effect on sharing discernment for concordant headlines (significant positive 3-way Treatment $\times$ Veracity $\times$ Concordance interaction); but that this moderation effect is driven by Democrats more so than Republicans (significant negative 4-way Treatment $\times$ Veracity $\times$ Concordance $\times$ Party interaction).

\begin{tabular}{|c|c|c|c|c|c|}
\hline \multirow[t]{2}{*}{ Simple effect } & \multirow[t]{2}{*}{ Net coefficient } & \multicolumn{2}{|c|}{$\begin{array}{l}\text { Participants that share political } \\
\text { content }\end{array}$} & \multicolumn{2}{|c|}{ All participants } \\
\hline & & S3 & S4 & S3 & S4 \\
\hline Treatment on false headlines & Treatment & 0.0002 & 0.0005 & 0.0117 & 0.0010 \\
\hline Treatment on true headlines & Treatment+Treatment $\times$ Veracity & 0.9185 & 0.6280 & 0.1535 & 0.1149 \\
\hline Veracity in Control & Veracity & 0.0083 & 0.0934 & 0.0237 & 0.0935 \\
\hline Veracity in Treatment & Veracity + Treatment $\times$ Veracity & $<.0001$ & 0.0001 & $<.0001$ & $<.0001$ \\
\hline
\end{tabular}

Table S3. P-values associated with the various simple effects from the regression models in Table S2. Despite the significant interactions with concordance and partisanship, sharing of false headlines was significantly lower in the Treatment than the Control for every combination of participant partisanship and headline concordance $(p<.05$ for all), with the exception of Republicans sharing concordant headlines when including all participants $(p=.36)$. 


\begin{tabular}{|c|c|c|c|c|}
\hline & \multicolumn{2}{|c|}{$\begin{array}{cc}(1) & (2) \\
\text { Participants that share political content }\end{array}$} & \multicolumn{2}{|c|}{$\begin{array}{l}(3) \\
\text { All participants }\end{array}$} \\
\hline & $\begin{array}{c}\begin{array}{c}\text { Controls } \\
\text { only }\end{array} \\
\end{array}$ & All conditions & $\begin{array}{c}\begin{array}{c}\text { Controls } \\
\text { only }\end{array} \\
\end{array}$ & $\begin{array}{c}\text { All } \\
\text { conditions } \\
\end{array}$ \\
\hline \multirow[t]{3}{*}{ Veracity $(0=$ False, $1=$ True $)$} & 0.00812 & 0.0163 & 0.0111 & 0.0154 \\
\hline & $(0.0262)$ & $(0.0234)$ & $(0.0206)$ & $(0.0212)$ \\
\hline & 0.756 & 0.486 & 0.589 & 0.466 \\
\hline \multirow[t]{3}{*}{ Active Control } & 0.00606 & & 0.0179 & \\
\hline & $(0.0303)$ & & $(0.0223)$ & \\
\hline & 0.841 & & 0.421 & \\
\hline \multirow[t]{3}{*}{ Active Control X Veracity } & 0.0155 & & 0.00856 & \\
\hline & $(0.0120)$ & & $(0.00660)$ & \\
\hline & 0.199 & & 0.195 & \\
\hline \multirow[t]{2}{*}{ Treatment } & & $\begin{array}{l}-0.0815^{* *} \\
(0.0261)\end{array}$ & & $\begin{array}{r}-0.0500^{* *} \\
(0.0185)\end{array}$ \\
\hline & & 0.00178 & & 0.00685 \\
\hline \multirow[t]{2}{*}{ Treatment X Veracity } & & $\begin{array}{l}0.0542 * * * \\
(0.0157)\end{array}$ & & $\begin{array}{r}0.0466^{* * *} \\
(0.00914)\end{array}$ \\
\hline & & 0.000538 & & $3.31 \mathrm{e}-07$ \\
\hline \multirow[t]{2}{*}{ Importance Treatment } & & -0.0504 & & -0.00966 \\
\hline & & $\begin{array}{c}(0.0274) \\
0.0660\end{array}$ & & $\begin{array}{c}(0.0193) \\
0.617\end{array}$ \\
\hline \multirow[t]{3}{*}{ Importance Treatment X Veracity } & & $0.0376 * *$ & & $0.0291 * * *$ \\
\hline & & $(0.0120)$ & & $(0.00634)$ \\
\hline & & 0.00178 & & $4.39 \mathrm{e}-06$ \\
\hline \multirow[t]{3}{*}{ Constant } & $0.477 * * *$ & $0.480 * * *$ & $0.359 * * *$ & $0.368 * * *$ \\
\hline & $(0.0227)$ & $(0.0160)$ & $(0.0166)$ & $(0.0127)$ \\
\hline & $<1 \mathrm{e}-10$ & $<1 \mathrm{e}-10$ & $<1 \mathrm{e}-10$ & $<1 \mathrm{e}-10$ \\
\hline Observations & 6,776 & 13,340 & 12,847 & 25,587 \\
\hline Participant clusters & 341 & 671 & 646 & 1286 \\
\hline Headline clusters & 20 & 20 & 20 & 20 \\
\hline R-squared & 0.001 & 0.007 & 0.001 & 0.004 \\
\hline
\end{tabular}

Table S4. Linear regressions predicting sharing intentions (1-6 Likert scale rescaled to [0,1]) in Study 5. Robust standard errors clustered on participant and headline. When comparing the passive and active controls, we see no significant main effect of condition or interaction with veracity, whether considering only participants who indicated that they sometimes consider sharing political content (Col 1) or all participants (Col 3). Therefore, as per our preregistered analysis plan, we collapse across control conditions for our main analysis. When comparing our main Treatment to the collapsed controls, we observed the predicted significant positive interaction between Treatment and news veracity, such that sharing discernment was higher in the Treatment compared to the controls, whether considering only participants who indicated that they sometimes consider sharing political content (Col 2) or considering all participants (Col 4). (Equivalent results are observed if comparing the Treatment only to the Active control.) When comparing our alternative Importance Treatment to the collapsed controls, we observed the predicted significant positive interaction between Importance Treatment and news veracity, such that sharing discernment was higher in the Importance Treatment compared to the controls, whether considering only participants who indicated that they sometimes consider sharing political content ( $\mathrm{Col} 2$ ) or considering all participants (Col 4). 


\begin{tabular}{|c|c|c|c|}
\hline Simple effect & Net coefficient & $\begin{array}{l}\text { Participants that } \\
\text { share political } \\
\text { content }\end{array}$ & $\begin{array}{c}\text { All } \\
\text { participants }\end{array}$ \\
\hline Treatment on false headlines & Treatment & 0.0018 & 0.0068 \\
\hline Treatment on true headlines & Treatment+Treatment $\times$ Veracity & 0.2411 & 0.8473 \\
\hline headlines & Importance Treatment & 0.0660 & 0.6166 \\
\hline $\begin{array}{l}\text { Importance Treatment on true } \\
\text { headlines }\end{array}$ & ImportanceTreatment+ImportanceTreatment $\times$ Veracity & 0.5883 & \\
\hline Veracity in Controls & Veracity & 0.4860 & 0.4665 \\
\hline Veracity in Treatment & Veracity + Treatment $\times$ Veracity & 0.0032 & 0.0027 \\
\hline Veracity in Importance Treatment & Veracity+ImportanceTreatment $\times$ Veracity & 0.0242 & 0.0470 \\
\hline
\end{tabular}

Table S5. P-values associated with the various simple effects from the regression models in Table S4. We observe the predicted significant negative simple effect of Treatment for false headlines, such that participants were less likely to consider sharing false headlines in the Treatment compared to the controls; and no significant simple effect of Treatment for true headlines, such that participants were no less likely to consider sharing true headlines in the Treatment compared to the controls. The negative simple effect of the Importance Treatment for false headlines was only marginally significant when considering sharer participants and non-significant when considering all participants, and the simple effect of Importance Treatment for true headlines was non-significant in both cases. Thus the results for the Importance Treatment are somewhat weaker than for the main Treatment. 


\section{Formal model of social media sharing based on limited attention and preferences}

Here we present a formal model to clearly articulate the competing hypotheses that we are examining. We then use this model to demonstrate the effectiveness of our experimental approach. Finally, we fit the model to our data in order to quantitatively support our inattentionbased account of misinformation sharing.

The modeling framework we develop here combines three lines of theory. The first is utility theory, which is the cornerstone of economic models of choice ${ }^{1-4}$. When people are choosing across a set of options (in our case, whether or not to share a given piece of content), they preferentially choose the option which gives them more utility, and the utility they gain for a given choice is defined by their preferences. In virtually all such models, preferences are assumed to be fixed (or at least to change over much longer timescales than that of any specific decision, e.g. months or years). The second line of theorizing involves importance of attention. A core tenet of psychological theory is that when attention is drawn to a particular dimension of the environment (broadly construed), that dimension tends to receive more weight in subsequent decisions ${ }^{5-8}$. While attention has been a primary focus in psychology, it has only recently begun to be integrated with utility theory models - such that attention can increase the weight put on certain preferences over others when making decisions ${ }^{9,10}$. Another major body of work documents how our cognitive capacities are limited (and our rationality is bounded) such that we are not able to bring all relevant pieces of information to bear on a given decision ${ }^{11-17}$. While the integration of cognitive constraints and utility theory is a core topic in behavioral economics, this approach has typically not been applied to attention and the implementation of preferences. Thus, we develop a model in which attention operates via cognitive constraints: agents are limited to only considering a subset of their preferences in any given decision, and attention determines which preferences are considered.

\subsection{Basic modeling framework}

Consider a piece of content $x$ which is defined by $k$ different characteristic dimensions; one of these dimensions is whether the content is false/misleading $F(x)$, and the other $k-1$ dimensions are non-accuracy-related (e.g. partisan alignment, humorousness, etc) defined as $C_{2}(x) \ldots C_{k}(x)$. In our model, the utility a given person expects to derive from sharing content $x$ is given by

$$
U(x)=-a_{1} \beta_{F} F(x)+\sum_{i=2}^{k} a_{i} \beta_{i} C_{i}(x)
$$

where $\beta_{F}$ indicates how much they dislike sharing misleading content and $\beta_{2} \ldots \beta_{k}$ indicate how much they care about each of the other dimensions (i.e. $\beta$ s indicate preferences); while $a_{1}$ indicates how much the person is paying attention to accuracy, and $a_{2} \ldots a_{k}$ indicate how much the person is paying attention to each of the other dimensions. The probability that the person chooses to share the piece of content $x$ is then increasing in $U(x)$. In the simplest decision rule, they will share if and only if $U(x)>0$; for a more realistic decision rule, one could use the logistic function, such that 


$$
p(\text { Share })=\frac{1}{1+e^{-\theta(U(x)+k)}}
$$

where $k$ determines the value of $U(x)$ at which the person is equally likely to share versus not share, and $\theta$ determines the steepness of the transition around that point from sharing to not sharing (the simple decision rule described in the previous sentence corresponds to $k=0, \theta \rightarrow$ Inf).

In the standard utility theory model, $a_{i}=1$ for all $i$ (all preferences are considered in every decision). In prior work on attention and preferences, $a$ values are continuous, and are determined by some feature of the choice - for example, in the context of economic decisions, the difference between minimum and maximum possible payoffs ${ }^{10}$, or the difference in percentage terms from the payoffs of other available lotteries ${ }^{9}$. Thus, all features are considered, but to differing degrees depending on how attention is focused.

In our limited-attention account, conversely, we incorporate cognitive constraints: we stipulate that people can consider only a subset of characteristic dimensions when making decisions. Specifically, agents can only attend to $m$ out of the $k$ utility terms in a given decision. That is, each value of $a$ is either 0 or $1, a_{i} \in\{0,1\}$; and because only $m$ terms can be considered at once, the $a$ values must sum to $k, \sum_{i=1}^{k} a_{i}=m$. Critically, the probability that any specific set of preference terms is attended to (i.e. which $a$ values are equal to 1) is heavily influenced by the situation, and (unlike preferences) can change from moment to moment - in response, for example, to the application of a prime. As described below in Section 3.7, we provide evidence that our limited-attention formulation fits the experimental data better than the framework used in prior models of attention and preferences where all preferences are considered but with differing weights (despite our model having an equal number of free parameters). It is also important to note that our basic formulation takes attention (i.e. the probability that a given set of $a_{i}$ values equal 1) as exogenously determined (e.g. by the context). However, in Section 3.7 we show that the results are virtually identical when using a more complex formulation where attention is also influenced by preferences, such that a person is more likely to pay attention to dimensions that they care more about (i.e. that have larger $\beta$ values).

\subsection{Preference-based versus inattention-based accounts}

Within this framework, we can articulate the preference-based versus inattention-based accounts. The preference-based account stipulates that people care less about accuracy than other factors when deciding what to share. This idea reflects that argument that many people have a low regard for the truth when deciding what to share on social media (e.g., Lewandowsky, Ecker, \& Cook, 2017). In terms of our model, this translates into the hypothesis that $\beta_{F}$ is small compared to one or more of the other $\beta$ terms - such that veracity has little impact on what content people decide to share (regardless of whether they are paying attention to it or not). Note that if the $\beta$ values on accuracy and political concordance, for example, were equal, then people would only be likely to share content that they judged to be both accurate and politically concordant. The preference-based sharing of false, politically concordant content thus requires a substantially higher $\beta$ on political concordance than on accuracy. 
Our inattention-based account, conversely, builds off the contention that people often consider only a subset of characteristic dimensions when making decisions. Thus, even if people do have a strong preference for accuracy (i.e. $\beta_{F}$ is as large, or larger than, other $\beta$ values), how accurate content is may still have little impact on what people decide to share if the context focuses their limited attention on other dimensions. The accuracy-based account of misinformation sharing, then, is the hypothesis that (i) $\beta_{F}$ is not appreciably smaller than the other $\beta$ values (e.g. the $\beta$ for political concordance), but that people nonetheless sometimes share misinformation because (ii) the probability of observing $a_{l}=1$ is far less than $1\left(p\left(a_{l}=1\right)<<1\right)$, such that people often fail to consider accuracy. As a result, the inattention-based account (but not the preference-based account) predicts that (iii) nudges that cause people to attend to accuracy can increase veracity's role in sharing by increasing the probability that $a_{l}=1\left(p\left(a_{l}=1\right) \mid\right.$ treatment $>p\left(a_{l}=1\right) \mid$ control $)$. That is, the accuracy nudge "shines an attentional spotlight" on the accuracy motive, increasing its chance to influence judgments.

\subsection{Application of model to our setting}

Next, we apply the general model presented in the previous section to the specific decision setting of our experiments. To do so, we consider $k=3$ content dimensions: to what extent the content seems inaccurate $(F ; 0=$ totally true to $1=$ totally false $)$, aligned with the user's partisanship ( $P$; from $0=$ totally misaligned to $1=$ totally aligned) or humorous $(H$, from $0=$ totally unfunny to $1=$ totally funny). There are, of course, numerous other relevant content dimensions that likely influence sharing which we do not include here; but in the name of tractability we focus on these dimensions as they are the dimensions that are manipulated in Studies 3 through 5 (article accuracy and partisanship are manipulated within-subjects in all experiments, accuracy focus is manipulated between-subjects in all experiments, and humor focus is manipulated between-subjects in Study 5). Below, we will demonstrate that modeling only these three dimensions allows us to characterize a large share of the variance in how often each headline gets shared; and look forward to future work building on the theoretical and experimental framework introduced here to explore a wider range of content dimensions.

We further stipulate that people are cognitively constrained to consider only $m=2$ of these dimensions in any given decision. We choose $m=2$ for the following reasons. First, the essence of the inattention-based account is that attention is limited, such that not all dimensions can be considered; thus, give that there are $k=3$ total dimensions, we necessarily choose a value of $m<3$ ( $m=3$ gives the standard utility theory model, which by definition cannot account for the accuracy priming effects we demonstrate in our experiments). We choose $m=2$ over $m=1$ because it seems overly restrictive to assume that people can only consider a single dimension in any given situation. Furthermore, below we demonstrate that $m=2$ yields a better fit to our experimental data than $m=1$.

\subsection{Analytic treatment of the effect of accuracy priming}

In this section, we determine the impact of priming accuracy predicted by the preference-based versus inattention-based accounts. We define $p$ as the probability that people do consider accuracy $\left(p\left(a_{l}=1\right)=p\right)$. For simplicity, we assume that the two cases in which accuracy is considered are equally likely, such that people consider accuracy and partisanship $\left(a_{1}=a_{2}=1\right.$ and 
$\left.a_{3}=0\right)$ with probability $p / 2$, and people consider accuracy and humor $\left(a_{1}=a_{3}=1\right.$ and $\left.a_{2}=0\right)$ with probability $p / 2$. Finally, with probability $1-p$, people do not consider accuracy and instead consider partisanship and humor $\left(a_{1}=0\right.$ and $\left.a_{2}=a_{3}=1\right)$. Also for simplicity, we use the simple decision rule whereby a piece of content $x$ is shared if and only if $U(x)>0$.

Within this setting, we can determine the probability that a given user (defined by her preferences $\beta_{F}, \beta_{P}$, and $\beta_{H}$, each of which is defined over the interval [-Inf,Inf]) shares a given piece of content $x$ (defined by its characteristics $F(x), C_{2}(x)$, and $C_{3}(x)$ ):

$$
\begin{aligned}
& \frac{p}{2} \mathbf{I}_{-\beta_{F} F(x)+\beta_{P} P(x)>0}+\frac{p}{2} \mathbf{I}_{-\beta_{F} F(x)+\beta_{H} H(x)>0}+(1-p) \mathbf{I}_{\beta_{P} P(x)+\beta_{H} H(x)>0} \\
& \begin{array}{ccc}
\text { (a) Considers accuracy \& partisanship } & \text { (b) Considers accuracy \& humor } & \text { (c) Considers partisanship \& humor } \\
\mathrm{a}_{1}=1, \mathrm{a}_{2}=1, \mathrm{a}_{3}=0 & \mathrm{a}_{1}=1, \mathrm{a}_{2}=0, \mathrm{a}_{3}=1 & \mathrm{a}_{1}=0, \mathrm{a}_{2}=1, \mathrm{a}_{3}=1
\end{array}
\end{aligned}
$$

The key question, then, is how the users' sharing decisions vary with $p$, the probability that users' attentional spotlight is directed at accuracy. In particular, imagine a piece of content that is aligned with the users' partisanship $P(x)=1$ and humorous $H(x)=1$, but false $F(x)=1$. When the user does not consider accuracy (term $c$ above, which occurs with probability 1- $p$ ), she will choose to share. When the user does consider accuracy (with probability $p$ ), her choice depends on her preferences. If $\beta_{F}<\beta_{P}$ and $\beta_{F}<\beta_{H}$ - that is, if the user cares about partisanship and humor more than accuracy, as per the preference-based account - she will still choose to share the misinformation. This is because the content's partisan alignment humorousness trumps its lack of accuracy, and therefore $p$ does not impact sharing. Thus, if the sharing of misinformation is driven by a true lack of concern about veracity relative to other factors - as per the preferencebased account - a manipulation that focuses attention on accuracy (and thereby increases $p$ ) will have no impact on the sharing of such misinformation.

If, on the other hand, $\beta_{F}>\beta_{P}$ and/or $\beta_{F}>\beta_{H}-$ that is, if the user cares about accuracy more than partisanship and/or humor - then directing attention at accuracy (and thereby increasing $p$ ) can influence sharing. If $\beta_{F}>\beta_{P}$, the user will choose not to share when considering accuracy and partisanship; and if $\beta_{F}>\beta_{H}$ the user will choose not to share when considering accuracy and humor. As a result, increasing $p$ will therefore decrease sharing. This scenario captures the essence of the inattention-based account.

Together, then, these two cases demonstrate how a manipulation that focuses attention on accuracy (increases $p$ ) - such as the manipulation in Studies 3 through 7 in the main text - will have differential impacts based on the relative importance the user places on accuracy. This illustrates how our experiments effectively disambiguate between the preference-based and inattention-based accounts of misinformation sharing.

This analysis also illustrates how drawing attention to a given dimension (e.g. priming it) need not translate into that dimension playing a bigger role in subsequent decisions. If the preference associated with that dimension is weak relative to the other dimensions (small $\beta$ ), then it will not drive choices even when attention is drawn to it. We will return to this observation when considering the lack of effect of the Active Control (priming humor) in Study 5. 


\subsection{Fitting the model to experimental data}

In the previous section, we provided a conceptual demonstration of how the accuracy priming effect we observe empirically in Studies 3 through 7 is consistent with the inattention-based account and inconsistent with the preference-based account. Here, we take this further by fitting the model to experimental data. This allows us to directly test the predictions of the two accounts regarding various model parameters described above in Section 3.2, and thus to provide direct evidence for the role of inattention versus preferences in the sharing of misinformation. Fitting the model to the data also allows us to test how well our model can account for the observed patterns of sharing.

To perform the fitting, we use the pretest data for Studies 4 and 5 to calculate the average perceived accuracy ("What is the likelihood that the above headline is true", from $1=$ Extremely unlikely to 7 = Extremely likely), political slant ("Assuming the above headline is entirely accurate, how favorable would it be to Democrats versus Republicans" from $1=$ More favorable for Democrats to $5=$ More favorable for Republicans), and humorousness ("In your opinion, is the above headline funny, amusing, or entertaining" from $1=$ Extremely unfunny to $7=$ Extremely funny) of each article. The pretest for the headlines in Studies 3 and 6 (both studies used the same items) did not include humorousness, and thus we cannot use those studies in the model fitting.

We must use the headline-level pretest ratings as a proxy for the ratings each individual would have of each article, because the participants in Studies 4 and 5 only made sharing decisions and did not rate each of the articles on perceived accuracy, political slant, or humorousness. Therefore, rather than separately estimating a model for every participant, we take a "representative agent" approach and estimate a single set of parameter values for the data averaged across subjects.

In order to define the political concordance $P(x)$ of headline $x$, however, it is necessary to consider Democrats and Republicans separately. This is because the extent to which a given headline is concordant for Democrats corresponds to the extent to which it is discordant for Republicans, and vice versa. Therefore, to create the dataset for fitting the model, the 44 total headlines (24 from Study 4 and 20 from Study 5) were each entered twice - once using the perceived accuracy ratings, humorousness ratings, and political slant ratings of Republicanleaning participants; and once using the perceived accuracy ratings, humorousness ratings, and 6 minus the political slant ratings (flipping the ratings to make them a measure of concordance) of Democratic-leaning participants. Each variable was scaled such that the minimum possible (rather than observed) value is 0 and the maximum possible (rather than observed) value is 1 . This therefore yielded a set of $88\{F(x), P(x), H(x)\}$ value triples. For each of these 88 data points, we also calculated the corresponding average sharing intention in the control and in the treatment. (For maximum comparability across the two studies, we used the passive control not the active control, and the treatment not the importance treatment, in Study 5.)

We then determined the set of parameter values that minimized the mean-squared error (difference between the observed data and the model predictions), using a somewhat more complicated formulation that uses the more realistic logistic function for the decision rule 
mapping from utility to choice, and allows each attentional case to have its own probability (rather than forcing the two cases that include accuracy to have the same probability):

$$
\begin{aligned}
& p \text { (share } \mid \text { control) } \\
& =p_{1 c} \frac{1}{1+\exp \left(-\theta\left(-\beta_{F} F(x)+\beta_{P} P(x)+k\right)\right)} \\
& +p_{2 c} \frac{1}{1+\exp \left(-\theta\left(-\beta_{F} F(x)+\beta_{H} H(x)+k\right)\right)}+\left(1-p_{1 c}\right. \\
& -p_{2 c)} \frac{1}{1+\exp \left(-\theta\left(\beta_{P} P(x)+\beta_{H} H(x)+k\right)\right)}
\end{aligned}
$$

and

$$
\begin{aligned}
& p(\text { share|treatment }) \\
& \qquad p_{1 t} \frac{1}{1+\exp \left(-\theta\left(-\beta_{F} F(x)+\beta_{P} P(x)+k\right)\right)} \\
& \quad+p_{2 t} \frac{1}{1+\exp \left(-\theta\left(-\beta_{F} F(x)+\beta_{H} H(x)+k\right)\right.}+\left(1-p_{1 t}\right. \\
& -p_{2 t)} \frac{1}{1+\exp \left(-\theta\left(\beta_{P} P(x)+\beta_{H} H(x)+k\right)\right)}
\end{aligned}
$$

Without loss of generality, as it is only the relative magnitude of the preference values that matters for choice, we fixed $\beta_{F}=1$ and determined the best-fitting values of the remaining 8 parameters $\left\{\beta_{P}, \beta_{H}, p_{1 c}, p_{2 c}, p_{1 t}, p_{2 t}, \theta, k\right\}$, subject to the constraints $p_{1 c}, p_{2 c}, p_{1 t}, p_{2 t} \geq 0$, $p_{1 c}, p_{2 c}, p_{1 t}, p_{2 t} \leq 1, p_{1 c}+p_{2 c} \leq 1$, and $p_{1 t}+p_{2 t} \leq 1$. We did this by comparing the predicted probability of sharing from the model with the average sharing intention for each of the headlinelevel data points, and minimizing the MSE using the interior-point algorithm (as implemented by the function fmincon in Matlab R2018b). We performed this optimization beginning from 100 randomly selected initial parameter sets, and kept the solution with the lowest MSE.

We use the comparison of treatment and control data to disentangle preferences ( $\beta$ values) from attention ( $p$-values). The key to our estimation strategy is that we hold the preference parameters $\beta_{F}, \beta_{P}$ and $\beta_{H}$ fixed across conditions while estimating different attention parameters in the control $\left(p_{1 c}, p_{2 c}\right)$ and the treatment $\left(p_{1 t}, p_{2 t}\right)$. As described above, fixed preferences is the standard assumption in virtually all utility theory models. Further evidence supporting the stability of the specifically relevant preference in our experiments comes from the observation, reported in the main text, that the treatment does not change participants' response to the postexperimental question about the importance of only sharing accurate content: If the treatment changed how much participants valued accuracy (rather than simply redirecting their attention), this would be likely to manifest itself as a greater reported valuation of accuracy.

We estimated the best-fit parameters separately for Studies 4 and 5. We did so for two reasons. First, the two studies were run with different populations (MTurk convenience sample versus Lucid quota-matched sample), so there is no reason to expect the best-fit parameter values to be the same. Second, because this analysis approach was not preregistered, we want to ensure that the results are replicable. Thus, we test the replicability of the results across the two studies, which differ in both the participants and the headlines used. 
Finally, we estimate confidence intervals for the best-fit parameter values and associated quantities of interest, as well as p-values for relevant comparisons, using bootstrapping. Specifically, we construct bootstrap samples separately for each study by randomly resampling participants with replacement. For each bootstrap sample, we then use the rating-level data for the participants in the bootstrap sample to calculate mean sharing intentions for each headline in control and treatment, and then refit the model using these new sharing intentions values. We store the resulting best-fit parameters derived from 1500 bootstrap samples, and use the 2.5 th percentile and 97.5th percentile of observed values to constitute the $95 \%$ confidence interval.

\subsection{Results}

We begin by examining the goodness of fit of our models, as the parameter estimates are only meaningful insomuch as the model does a good job of predicting the data. As mean-squared error (Study 4, MSE = 0.0036; Study 5, MSE = 0.0046) is not easily interpretable, we also consider the correlation between the model predictions and the observed average sharing intentions for each headline within each partisanship group (Democrats vs Republicans) in each experimental condition. As shown in Figure S1, we observe a high correlation in both Study 4, $r=0.862$, and Study 5, $r=0.797$. This indicates that despite only considering three of the many possible content dimensions, our model specification is able to capture much of the dynamics of sharing intentions observed in our experiments.
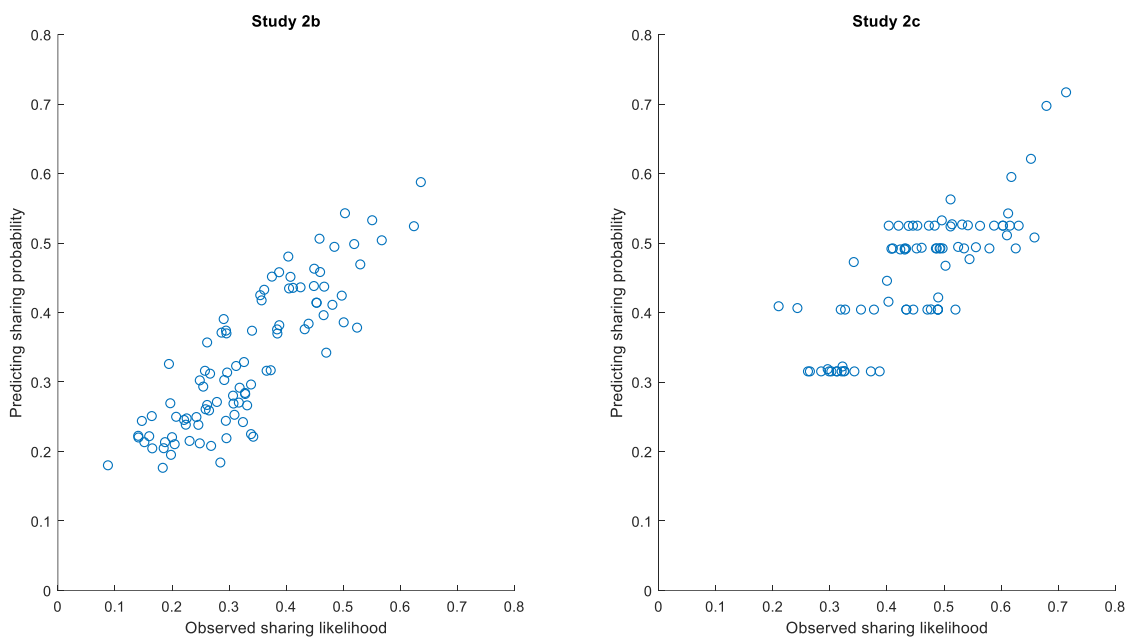

Figure S1. Observed and predicted sharing in Studies 4 and 5.

We now turn to the parameter estimates themselves. For each study, Extended Data Table 1 shows the best-fit parameter values; the overall probability that participants consider accuracy $\left(p_{1 c}+p_{2 c}\right)$, political concordance $\left(p_{1 c}+\left(1-p_{1 c}-p_{2 c}\right)\right)$, and humorousness $\left(p_{2 c}+\left(1-p_{1 c}-p_{2 c}\right)\right)$ in the control; the overall probability that participants consider accuracy $\left(p_{1 t}+p_{2 t}\right)$, political concordance $\left(p_{1 t}+\left(1-p_{1 t}-p_{2 t}\right)\right)$, and humorousness $\left(p_{2 t}+\left(1-p_{1 t}-p_{2 t}\right)\right)$ in the treatment; and the treatment effect on each of those quantities (probability in treatment minus probability in control). Note that because the best-fit values for $\beta_{\mathrm{H}}$ are substantially smaller than $\beta_{\mathrm{F}}(=1)$ and $\beta_{\mathrm{P}}-$ that is, because participants don't put much value on humorousness - the estimates for probability of considering humorousness are not particularly meaningful. This is because even if participants did pay attention to humorousness, it would always be outweighed by whichever other factor was being 
considered; and thus it is not possible from the choice data to precisely determine whether humorousness was attended to; this is not problematic for us, however, as none of the key predictions involve probability of attending to humorousness.

There are three key results in Extended Data Table 1. First, inconsistent with the preferencebased account, the best-fit preference parameters indicate that participants value accuracy as much as or more than partisanship. Thus, they would be unlikely to share false but politically concordant content if they were attending to accuracy and partisanship. (This is not to say that partisanship is unimportant, but rather that partisanship does not override accuracy ideologically aligned content must also be sufficiently accurate in order to have a high sharing probability). Second, the best-fit attention parameters indicate participants often fail to consider accuracy because their attention is directed to other content dimensions. This can lead them to share content that they would have assessed as inaccurate (and chosen not to share), had they considered accuracy. And finally, the Treatment increases participants' likelihood of considering accuracy (and thereby reduces the sharing of false statements).

\subsection{Alternative model specifications}

In this section, we compare the performance of our model to various alternative specifications. First, we contrast our assumption that participants can attend to $m=2$ of the $k=3$ content dimensions in any given decision with a model in which $m=1$ (i.e. where participants can only consider one dimension per decision). This yields the following formulation:

$$
\begin{aligned}
& p(\text { share } \mid \text { control }) \\
& =p_{1 c} \frac{1}{1+\exp \left(-\theta\left(-\beta_{F} F(x)+k\right)\right)}+p_{2 c} \frac{1}{1+\exp \left(-\theta\left(\beta_{P} P(x)+k\right)\right)}+\left(1-p_{1 c}\right. \\
& -p_{2 c)} \frac{1}{1+\exp \left(-\theta\left(\beta_{H} H(x)+k\right)\right)}
\end{aligned}
$$

and

$$
\begin{aligned}
& p(\text { share|treatment }))) \\
& \qquad p_{1 t} \frac{1}{1+\exp \left(-\theta\left(-\beta_{F} F(x)+k\right)\right)}+p_{2 t} \frac{1}{1+\exp \left(-\theta\left(\beta_{P} P(x)+k\right)\right)}+\left(1-p_{1 t}\right. \\
& -p_{2 t)} \frac{1}{1+\exp \left(-\theta\left(\beta_{H} H(x)+k\right)\right)}
\end{aligned}
$$

Since this formulation has the same number of free parameters as the main $m=2$ model, it is straightforward to compare model fit by simply asking which model fits the data better. Fitting this model to the data yields a higher mean-squared error than our main model with $m=2$ in both Study 4 (MSE $=0.0043$ vs MSE=0.0036 in the $m=2$ model) and Study 5 (MSE=0.0056 vs $\mathrm{MSE}=0.0046$ in the $m=2$ model), indicating that the $m=2$ model is preferable.

Next, we contrast our model - based on cognitive constraints - with the formulation used in prior models of attention and preferences ${ }^{9,10}$ in which all preferences are considered in every decision, but are differentially weighted by attention. This alternative approach yields the following formulation: 
$p($ share $\mid$ control $)=\frac{1}{1+\exp \left(-\theta\left(-p_{1 c} \beta_{F} F(x)+p_{2 c} \beta_{P} P(x)+\left(1-p_{1 c}-p_{2 c}\right) \beta_{H} H(x)+k\right)\right)}$

and

$p($ share $\mid$ treatment $)=\frac{1}{1+\exp \left(-\theta\left(-p_{1 t} \beta_{F} F(x)+p_{2 t} \beta_{P} P(x)+\left(1-p_{1 t}-p_{2 t}\right) \beta_{H} H(x)+k\right)\right)}$

Once again, this alternative formulation has the same number of free parameters as our main model, allowing for straightforward model comparison. Fitting this model to the data yields a higher mean-squared error than our main model in both Study 4 (MSE=0.0039 vs MSE=0.0036 in the main model) and Study $5(\mathrm{MSE}=0.0057 \mathrm{vs} \mathrm{MSE}=0.0046$ in the main model), indicating that the main model is preferable.

Next, we examine the simplifying assumption in our main model that attention (i.e. the probability that any given content dimension is considered) is exogenously determine (e.g. by the context). In reality, one's preferences may also influence how one allocates one's attention. For example, a person who cares a great deal about accuracy may be more likely to attend to accuracy. To consider the consequences of such a dependence, we additionally weight each attention scenario not just be its associated value of $p\left(p_{1 c}, p_{2 c}\right.$, etc.) but also by the relative preference weight put on the two dimensions considered in that scenario. This yields the following formulation:

$p($ share $\mid$ control $)$

$$
\begin{aligned}
& =p_{1 c} \frac{\beta_{F}+\beta_{P}}{\pi_{c}} \frac{1}{1+\exp \left(-\theta\left(-\beta_{F} F(x)+k\right)\right)} \\
& +p_{2 c} \frac{\beta_{F}+\beta_{H}}{\pi_{c}} \frac{1}{1+\exp \left(-\theta\left(\beta_{P} P(x)+k\right)\right)}+\left(1-p_{1 c}\right. \\
& \left.-p_{2 c}\right) \frac{\beta_{P}+\beta_{H}}{\pi_{c}} \frac{1}{1+\exp \left(-\theta\left(\beta_{H} H(x)+k\right)\right)}
\end{aligned}
$$

and

$$
\begin{aligned}
& p(\text { share|treatment }) \\
& \qquad p_{1 t} \frac{\beta_{F}+\beta_{P}}{\pi_{t}} \frac{1}{1+\exp \left(-\theta\left(-\beta_{F} F(x)+k\right)\right)} \\
& +p_{2 t} \frac{\beta_{F}+\beta_{H}}{\pi_{t}} \frac{1}{1+\exp \left(-\theta\left(\beta_{P} P(x)+k\right)\right)}+\left(1-p_{1 t}\right. \\
& \left.-p_{2 t}\right) \frac{\beta_{P}+\beta_{H}}{\pi_{t}} \frac{1}{1+\exp \left(-\theta\left(\beta_{H} H(x)+k\right)\right)}
\end{aligned}
$$

where $\pi_{c}$ and $\pi_{t}$ are normalization constants that force the probabilities to sum to one, such that

$$
\begin{aligned}
& \pi_{c}=p_{1 c}\left(\beta_{F}+\beta_{P}\right)+p_{2 c}\left(\beta_{F}+\beta_{H}\right)+\left(1-p_{1 c}-p_{2 c}\right)\left(\beta_{P}+\beta_{H}\right) \\
& \pi_{t}=p_{1 t}\left(\beta_{F}+\beta_{P}\right)+p_{2 c}\left(\beta_{F}+\beta_{H}\right)+\left(1-p_{1 c}-p_{2 c}\right)\left(\beta_{P}+\beta_{H}\right)
\end{aligned}
$$

Once again, this model has the same number of free parameters as the main model. Unlike the previous alternative models, this model of endogenous attention fits the data exactly as well as 
the main (exogenous attention) model (identical MSE to 4 decimal places in both studies). The resulting fits have identical preference values of $\beta_{P}$ and $\beta_{H}$ (and therefore contradict the preference-based account in the same way as the main model) and qualitatively similar results regarding the attention parameters: in the control, participants often fail to consider accuracy (overall probability of considering accuracy $=0.07$ in Study 4, 0.61 in Study 5), and the treatment increases participants' probability of considering accuracy (by 0.02 in Study 4, and 0.08 in Study 5). Furthermore, an analytic treatment of this model provides equivalent results to the analysis of the exogenous attention model presented above in Section 3.4. 


\section{Ethics of Digital Field Experimentation}

Field experimentation, such as our Study 7, necessarily involves engaging in people's natural activities to assess the effect of a treatment in situ. As digital experimentation on social media becomes more attractive to social scientists, there are increasing ethical considerations that must be taken into account ${ }^{19-21}$.

One such consideration is the nature of the interaction between Twitter users and our bot accounts. As discussed above, this involved following individuals who shared links to misinformation sites, and then sending a DM to those individuals who followed our bot accounts back. We believe that the potential harm of an account following and sending a DM to an individual is minimal; and that the potential benefits of scientific understanding and an increase in shared news quality outweigh that negligible risk. Both the Yale University Committee for the Use of Human Subjects (IRB protocol \#2000022539) and the MIT COUHES (Protocol \#1806393160) agreed with our assessment. With regard to informed consent, it is standard practice in field experiments to eschew informed consent because much of the value of field experiments comes from participants not knowing they are in an experiment (thus providing ecological validity). As obtaining informed consent would disrupt the user's normal experience using Twitter, and greatly reduce the validity of the design - and the risks were minimal - both institutional review boards waived the need for informed consent. A final consideration is the ethical collection of individuals' tweet histories for analysis. Since we are only considering publicly available tweets, and hence any collated dataset would be the product of secondary research, we believe this to be an acceptable practice.

There is the open question of how these considerations interact, and if practices that are separately appropriate can create ethically ambiguous situations when conducted conjointly. Data rights on social media are a complicated and ever-changing social issue with no clear answers. We hope Study 7 highlights some principles and frameworks for considering these issues in the context of digital experimentation, and helps create more discussion and future work on concretely establishing norms of engagement.

There has been some discussion about the ethics of nudges, primes, modifications to choice architectures, and other interventions for digital behavior change. Some worry that these interventions can be paternalistic, and favor the priorities of platform designers over users. Our intervention - making the concept of accuracy salient - does not prescribe any agenda or normative stance to users. We do not tell users what is accurate versus inaccurate, or even tell them that they should be taking accuracy into account when sharing. Rather, the intervention simply moves the spotlight of attention towards accuracy, and then allows the user to make their own determination of accuracy and make their own choice about how to act on that determination.

While we believe this intervention is ethically sound, we also acknowledge the fact that if this methodology was universalized as a new standard for social science research, it could further dilute and destabilize the Twitter ecosystem, which already suffers from fake accounts, spam, and misinformation. Future work should invest in new frameworks for digital experimentation that maintains social media's standing as a town square for communities to genuinely engage in communication, while also allowing researchers to causally understand user behavior on the platform. These frameworks may involve, for example, external software libraries built on top of publicly available APIs, or explicit partnerships with the social media companies themselves. 


\section{Additional Analysis for Study 7}

Table S6 shows a consistent significant interaction between treatment and the tweet being an RTwithout-comment, such that the treatment consistently increases the average quality of RTswithout-comment but has no significant effect on primary tweets. (We do not conduct this interaction analysis for summed relative quality or discernment, because the differences in tweet volume between RTs-without-comment and primary tweets makes those measures not comparable.)

\begin{tabular}{|c|c|c|c|c|c|c|c|c|c|c|c|}
\hline \multirow{2}{*}{$\begin{array}{c}\text { Randomization- } \\
\text { Failure }\end{array}$} & Article & Model & \multicolumn{3}{|c|}{ Interaction } & \multicolumn{3}{|c|}{ Simple effect on NRT } & \multicolumn{2}{|c|}{ Simple effect on RT } \\
\cline { 6 - 11 } & Type & Spec & Coeff & Reg p & FRI p & Coeff & Reg p & FRI p & Coeff & Reg p & FRI p \\
\hline ITT & All & Wave FE & 0.008 & $\mathbf{0 . 0 0 4}$ & $\mathbf{0 . 0 0 3}$ & 0.000 & 0.741 & 0.701 & 0.007 & $\mathbf{0 . 0 0 3}$ & $\mathbf{0 . 0 0 4}$ \\
\hline ITT & All & Wave PS & 0.008 & $\mathbf{0 . 0 0 6}$ & $\mathbf{0 . 0 0 3}$ & 0.000 & 0.761 & 0.756 & 0.007 & $\mathbf{0 . 0 0 4}$ & $\mathbf{0 . 0 0 4}$ \\
\hline ITT & All & Date FE & 0.007 & $\mathbf{0 . 0 2 2}$ & $\mathbf{0 . 0 0 4}$ & -0.001 & 0.725 & 0.800 & 0.006 & $\mathbf{0 . 0 1 7}$ & $\mathbf{0 . 0 1 4}$ \\
\hline ITT & All & Date PS & 0.007 & $\mathbf{0 . 0 3 1}$ & $\mathbf{0 . 0 0 6}$ & -0.001 & 0.725 & 0.703 & 0.006 & $\mathbf{0 . 0 2 7}$ & $\mathbf{0 . 0 1 2}$ \\
\hline Exclude & All & Wave FE & 0.008 & $\mathbf{0 . 0 0 6}$ & $\mathbf{0 . 0 0 4}$ & -0.001 & 0.676 & 0.635 & 0.007 & $\mathbf{0 . 0 0 4}$ & $\mathbf{0 . 0 0 9}$ \\
\hline Exclude & All & Wave PS & 0.008 & $\mathbf{0 . 0 0 7}$ & $\mathbf{0 . 0 0 4}$ & -0.001 & 0.690 & 0.687 & 0.007 & $\mathbf{0 . 0 0 5}$ & $\mathbf{0 . 0 0 8}$ \\
\hline Exclude & All & Date FE & 0.006 & $\mathbf{0 . 0 3 3}$ & $\mathbf{0 . 0 0 7}$ & -0.001 & 0.629 & 0.740 & 0.006 & $\mathbf{0 . 0 3 2}$ & $\mathbf{0 . 0 3 2}$ \\
\hline Exclude & All & Date PS & 0.006 & $\mathbf{0 . 0 4 0}$ & $\mathbf{0 . 0 0 9}$ & -0.001 & 0.629 & 0.653 & 0.006 & $\mathbf{0 . 0 4 2}$ & $\mathbf{0 . 0 2 3}$ \\
\hline ITT & No Opinion & Wave FE & 0.009 & $\mathbf{0 . 0 0 1}$ & $\mathbf{0 . 0 0 1}$ & -0.001 & 0.466 & 0.453 & 0.008 & $\mathbf{0 . 0 0 1}$ & $\mathbf{0 . 0 0 3}$ \\
\hline ITT & No Opinion & Wave PS & 0.009 & $\mathbf{0 . 0 0 1}$ & $\mathbf{0 . 0 0 1}$ & -0.001 & 0.454 & 0.491 & 0.008 & $\mathbf{0 . 0 0 2}$ & $\mathbf{0 . 0 0 4}$ \\
\hline ITT & No Opinion & Date FE & 0.008 & $\mathbf{0 . 0 0 7}$ & $\mathbf{0 . 0 0 1}$ & -0.001 & 0.458 & 0.646 & 0.007 & $\mathbf{0 . 0 0 9}$ & $\mathbf{0 . 0 1 3}$ \\
\hline ITT & No Opinion & Date PS & 0.008 & $\mathbf{0 . 0 0 9}$ & $\mathbf{0 . 0 0 1}$ & -0.001 & 0.458 & 0.493 & 0.007 & $\mathbf{0 . 0 1 3}$ & $\mathbf{0 . 0 0 7}$ \\
\hline Exclude & No Opinion & Wave FE & 0.009 & $\mathbf{0 . 0 0 1}$ & $\mathbf{0 . 0 0 2}$ & -0.001 & 0.476 & 0.486 & 0.008 & $\mathbf{0 . 0 0 1}$ & $\mathbf{0 . 0 0 9}$ \\
\hline Exclude & No Opinion & Wave PS & 0.009 & $\mathbf{0 . 0 0 2}$ & $\mathbf{0 . 0 0 1}$ & -0.001 & 0.450 & 0.505 & 0.008 & $\mathbf{0 . 0 0 3}$ & $\mathbf{0 . 0 0 8}$ \\
\hline Exclude & No Opinion & Date FE & 0.007 & $\mathbf{0 . 0 1 3}$ & $\mathbf{0 . 0 0 2}$ & -0.001 & 0.442 & 0.655 & 0.006 & $\mathbf{0 . 0 1 7}$ & $\mathbf{0 . 0 2 9}$ \\
\hline Exclude & No Opinion & Date PS & 0.008 & $\mathbf{0 . 0 1 5}$ & $\mathbf{0 . 0 0 1}$ & -0.001 & 0.442 & 0.495 & 0.006 & $\mathbf{0 . 0 2 1}$ & $\mathbf{0 . 0 1 4}$ \\
\hline
\end{tabular}

Table S6. Coefficients and p-values associated with the interaction between treatment and tweet type, and each simple effect of treatment, when predicting average relative quality for Study 7 . In the model specification column, FE represents fixed effects (i.e. just dummies) and PS represents post-stratification (i.e. centered dummies interacted with the post-treatment dummy). P-values below 0.05 are bolded. 
The analyses presented in Extended Data Table 4 collapse across waves to maximize statistical power. As evidence that this aggregation is justified, we examine the models in which the treatment effect is post-stratified on wave (i.e. the wave dummies are interacted with the posttreatment dummy). Table S7 shows the p-values generated by a joint significance test over the wave-post-treatment interactions (i.e. testing whether the treatment effect differed significantly in size across waves) for the four dependent variables crossed with the four possible inclusion criteria choices. As can be seen, in all cases the joint significance test is extremely far from significant. This lack of significant interaction between treatment and wave supports our decision to aggregate the data across waves.

\begin{tabular}{|l|l|l|l|r|}
\hline Tweet Type & $\begin{array}{l}\text { Randomization- } \\
\text { Failure }\end{array}$ & $\begin{array}{l}\text { Average } \\
\text { Relative } \\
\text { Quality }\end{array}$ & $\begin{array}{l}\text { Summed } \\
\text { Relative } \\
\text { Quality }\end{array}$ & Discernment \\
\hline All & Exclude & 0.685 & 0.378 & 0.559 \\
\hline All & ITT & 0.743 & 0.313 & 0.613 \\
\hline RT & Exclude & 0.710 & 0.508 & 0.578 \\
\hline RT & ITT & 0.722 & 0.535 & 0.687 \\
\hline
\end{tabular}

Table S7. P-values generated by a joint significant test of the interaction between wave 2 and post-treatment and wave 3 and post-treatment, from the models in Extended Data Table 4 where treatment effect is post-stratified on wave. 
Next, Table S8 shows models testing for an interaction between the treatment and the user's number of followers (log-transformed due to extreme right skew) when predicting average relative quality of tweets. As can be seen, none of the interactions are significant, and the sign of all interactions is positive. Thus, there is no evidence that the treatment is less effective for users with more followers. If anything, the effect is directionally in the opposite direction.

\begin{tabular}{|c|c|c|c|c|c|c|}
\hline $\begin{array}{l}\text { Tweet } \\
\text { Type }\end{array}$ & Article Type & $\begin{array}{l}\text { Randomization- } \\
\text { Failure }\end{array}$ & $\begin{array}{l}\text { Model } \\
\text { Spec } \\
\end{array}$ & Coeff & $\operatorname{Reg} \mathrm{p}$ & FRI $p$ \\
\hline All & All & ITT & Wave FE & 0.003 & 0.252 & 0.905 \\
\hline All & All & ITT & Wave PS & 0.003 & 0.200 & 0.123 \\
\hline All & All & ITT & Date FE & 0.002 & 0.360 & 0.301 \\
\hline All & All & ITT & Date PS & 0.002 & 0.468 & 0.441 \\
\hline RT & All & ITT & Wave FE & 0.002 & 0.364 & 0.919 \\
\hline RT & All & ITT & Wave PS & 0.002 & 0.319 & 0.201 \\
\hline RT & All & ITT & Date FE & 0.002 & 0.468 & 0.375 \\
\hline RT & All & ITT & Date PS & 0.002 & 0.452 & 0.455 \\
\hline All & All & Exclude & Wave FE & 0.004 & 0.143 & 0.977 \\
\hline All & All & Exclude & Wave PS & 0.004 & 0.124 & 0.066 \\
\hline All & All & Exclude & Date FE & 0.003 & 0.225 & 0.152 \\
\hline All & All & Exclude & Date PS & 0.003 & 0.357 & 0.324 \\
\hline RT & All & Exclude & Wave FE & 0.003 & 0.215 & 0.979 \\
\hline RT & All & Exclude & Wave PS & 0.003 & 0.204 & 0.111 \\
\hline RT & All & Exclude & Date FE & 0.003 & 0.307 & 0.200 \\
\hline RT & All & Exclude & Date PS & 0.003 & 0.345 & 0.354 \\
\hline All & No Opinion & ITT & Wave FE & 0.003 & 0.190 & 0.954 \\
\hline All & No Opinion & ITT & Wave PS & 0.004 & 0.167 & 0.121 \\
\hline All & No Opinion & ITT & Date FE & 0.003 & 0.285 & 0.216 \\
\hline All & No Opinion & ITT & Date PS & 0.002 & 0.380 & 0.386 \\
\hline RT & No Opinion & ITT & Wave FE & 0.002 & 0.296 & 0.956 \\
\hline RT & No Opinion & ITT & Wave PS & 0.003 & 0.269 & 0.202 \\
\hline RT & No Opinion & ITT & Date FE & 0.002 & 0.403 & 0.334 \\
\hline RT & No Opinion & ITT & Date PS & 0.002 & 0.371 & 0.458 \\
\hline All & No Opinion & Exclude & Wave FE & 0.004 & 0.098 & 0.986 \\
\hline All & No Opinion & Exclude & Wave PS & 0.004 & 0.097 & 0.064 \\
\hline All & No Opinion & Exclude & Date FE & 0.004 & 0.161 & 0.094 \\
\hline All & No Opinion & Exclude & Date PS & 0.003 & 0.275 & 0.286 \\
\hline RT & No Opinion & Exclude & Wave FE & 0.003 & 0.179 & 0.984 \\
\hline RT & No Opinion & Exclude & Wave PS & 0.003 & 0.178 & 0.128 \\
\hline RT & No Opinion & Exclude & Date FE & 0.003 & 0.264 & 0.173 \\
\hline RT & No Opinion & Exclude & Date PS & 0.003 & 0.283 & 0.375 \\
\hline
\end{tabular}

Table S8. Coefficients and p-values associated with the interaction between treatment and log(\# followers) each model predicting average relative quality for Study 3. In the model specification column, FE represents fixed effects (i.e. just dummies) and PS represents post-stratification (i.e. centered dummies interacted with the post-treatment dummy). All p-values are above 0.05. 
Finally, as shown in Table S9, we see no evidence of a treatment effect when considering tweets that did not contain links to any of the rated news sites, or when considering the probability that any rated tweets occurred.

\begin{tabular}{|l|l|l|l|l|l|l|l|r|}
\hline \multirow{2}{*}{ Tweet Type } & \multirow{2}{*}{$\begin{array}{c}\text { Randomization- } \\
\text { Failure }\end{array}$} & \multirow{2}{*}{$\begin{array}{c}\text { Model } \\
\text { Spec }\end{array}$} & \multicolumn{2}{|c|}{ Tweets without rated links } & \multicolumn{4}{c|}{ Any rated tweets } \\
\cline { 5 - 10 } & & Coeff & Reg p & \multicolumn{1}{l|}{ FRI p } & \multicolumn{1}{l|}{ Coeff } & Reg p & FRI p \\
\hline All & ITT & Wave FE & 0.492 & 0.483 & 0.342 & 0.004 & 0.600 & 0.602 \\
\hline All & ITT & Wave PS & 0.364 & 0.577 & 0.460 & 0.004 & 0.611 & 0.590 \\
\hline All & ITT & Date FE & 0.160 & 0.843 & 0.788 & 0.006 & 0.472 & 0.494 \\
\hline All & ITT & Date PS & 0.126 & 0.873 & 0.825 & 0.010 & 0.293 & 0.181 \\
\hline All & Exclude & Wave FE & 0.221 & 0.756 & 0.672 & -0.001 & 0.890 & 0.894 \\
\hline All & Exclude & Wave PS & 0.150 & 0.823 & 0.763 & 0.000 & 0.978 & 0.979 \\
\hline All & Exclude & Date FE & -0.232 & 0.779 & 0.697 & 0.001 & 0.929 & 0.929 \\
\hline All & Exclude & Date PS & -0.127 & 0.876 & 0.827 & 0.006 & 0.500 & 0.397 \\
\hline RT & ITT & Wave FE & 0.440 & 0.408 & 0.266 & -0.001 & 0.917 & 0.895 \\
\hline RT & ITT & Wave PS & 0.332 & 0.495 & 0.367 & -0.002 & 0.806 & 0.760 \\
\hline RT & ITT & Date FE & 0.246 & 0.687 & 0.569 & 0.001 & 0.943 & 0.927 \\
\hline RT & ITT & Date PS & 0.139 & 0.814 & 0.744 & 0.004 & 0.657 & 0.560 \\
\hline RT & Exclude & Wave FE & 0.266 & 0.620 & 0.505 & -0.006 & 0.455 & 0.338 \\
\hline RT & Exclude & Wave PS & 0.197 & 0.692 & 0.600 & -0.006 & 0.450 & 0.346 \\
\hline RT & Exclude & Date FE & -0.004 & 0.995 & 0.992 & -0.005 & 0.599 & 0.510 \\
\hline RT & Exclude & Date PS & -0.028 & 0.963 & 0.946 & 0.001 & 0.928 & 0.907 \\
\hline
\end{tabular}

Table S9. Coefficients and p-values associated with each model predicting number of unrated tweets and presence of any rated tweets for Study 7. In the model specification column, FE represents fixed effects (i.e. just dummies) and PS represents post-stratification (i.e. centered dummies interacted with the post-treatment dummy). 
Turning to visualization, in Figure $\mathrm{S} 2$ we show the results of domain-level analyses. These analyses compute the fraction of pre-treatment rated links that link to each of the 60 rated domains, and the fraction of rated links in the 24 hours post-treatment that link to each of the 60 rated domains. For each domain, we then plot the difference between these two fractions on the $\mathrm{y}$-axis, and the fact-checker trust rating from Pennycook \& Rand ${ }^{22}$ on the $\mathrm{x}$-axis.
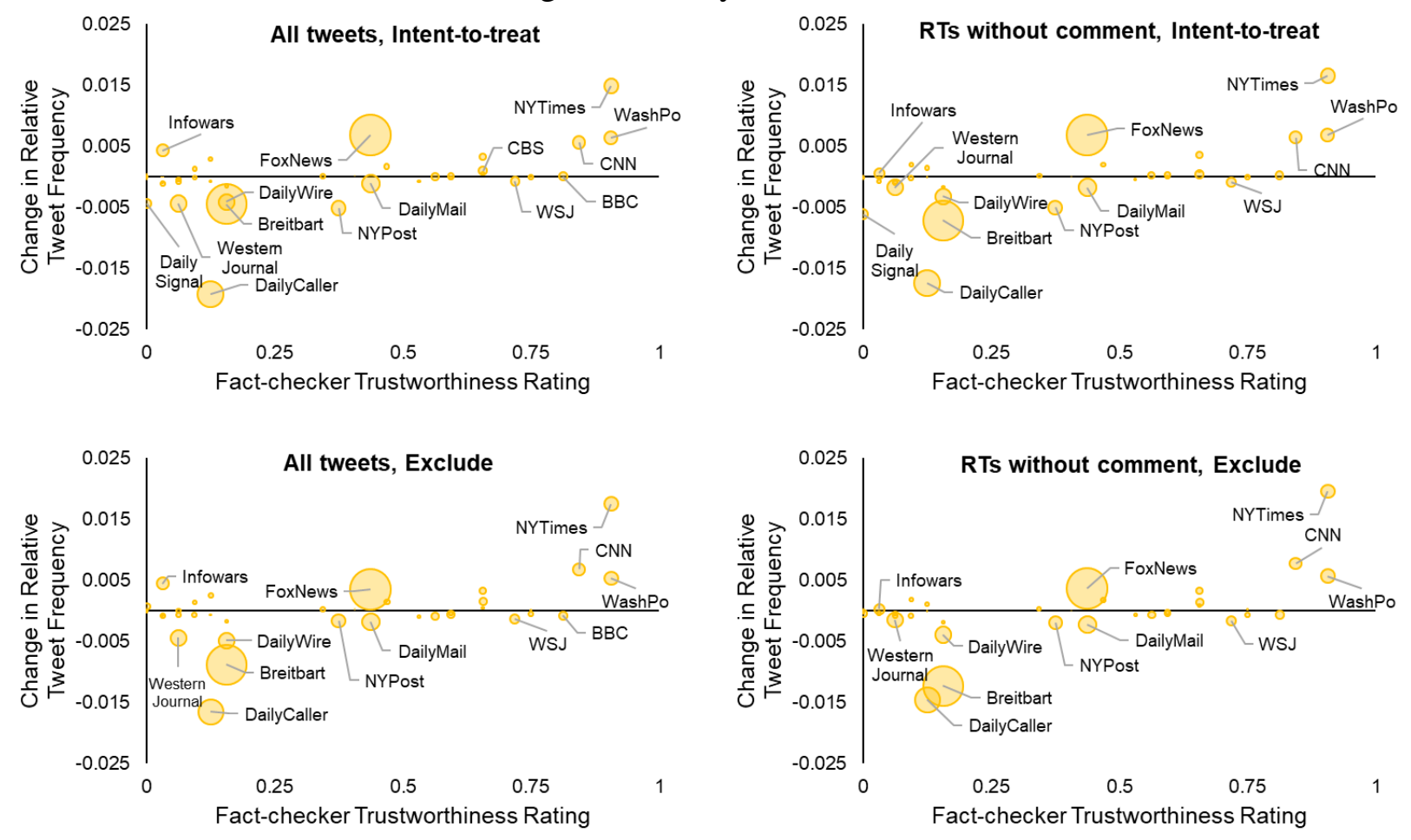

Figure S2. Domain-level analysis for each combination of approach to randomization failure (exclusion or intent-to-treat) and tweet type (all or only RTs-without-comment). Size of dots is proportional to pre-treatment tweet count. Outlets with at least 500 pre-treatment tweets are labeled. 


\section{Modeling the spread of misinformation}

Our paper theoretically and empirically investigates the role of accuracy and inattention in individuals' decisions about what to share online. To investigate how these individual-level choices - and the accuracy nudge interventions we introduce to improve such choices - translate into population-level outcomes regarding the spread of misinformation, we employ simulations of social spreading dynamics. The key goal of the simulations is to shed light on how network effects either suppress or amplify the impact of the accuracy intervention (which we have shown to improve individual choices).

In our simulations, a population of agents is embedded in a network. When an agent is first exposed to a piece of information, they share it with probability $s$. Based on the Control conditions of Study 3-6, we take the probability of sharing a piece of fake news at baseline (i.e., without intervention) to be approximately $s=0.3$. The Full Attention Treatment of Study 6 indicates that if an intervention was able to entirely eliminate inattention, the probability of sharing would be reduced by $50 \%$. Thus, we vary $s$ across the interval $[0.15,0.3]$ and examine the impact on the spread of misinformation. If an agent does choose to share a piece of information, each of their followers is exposed to that information with probability $p(p<<1$, as most shared content is never seen because it is quickly pushed down the newsfeed queue ${ }^{23}$; we use $p=0.1$ ).

In each run of the simulation, a piece of misinformation is seeded in the network by randomly selecting an initial user to be exposed to that misinformation. They then decide whether to share based on $s$, if they do then each of their followers is exposed with probability $p$; then each of the exposed followers shares with probability $s$, and if so then their followers are exposed with probability $p$, and so on. The simulation then runs until no new exposures occur, and the total fraction of the population exposed to the piece of information across the simulation run is calculated.

This procedure thus allows us to determine how a given decrease in individuals' probability of sharing misinformation impacts the population-level outcome of misinformation spread. We examine how the fraction of agents that get exposed varies with the magnitude of the intervention effect (extent to which $s$ is reduced), the type of network structure (cycle, WattsStrogatz small-world network with rewiring rate of 0.1, or Barabási-Albert scale-free network), and the density of the network (average number of neighbors $k$ ). Our simulations use a population of size $N=1000$, and we show the average result of 10,000 simulation runs for each set of parameter values.

Extended Data Figure 6 shows how a given percentage reduction in individual sharing probability (between 0 and 50\%) translates into a percentage reduction in the fraction of the population that is exposed to the piece of misinformation. 


\section{Supplementary references}

1. Fishburn, P. C. Utility Theory. Manage. Sci. 14, 335-378 (1968).

2. Stigler, G. J. The Development of Utility Theory. I. J. Polit. Econ. 58, 307-327 (1950).

3. Quiggin, J. A theory of anticipated utility. J. Econ. Behav. Organ. 3, 323-343 (1982).

4. Barberis, N. C. Thirty years of prospect theory in economics: A review and assessment. Journal of Economic Perspectives 27, 173-196 (2013).

5. Taylor, S. E. \& Thompson, S. C. Stalking the elusive 'vividness' effect. Psychol. Rev. 89, 155-181 (1982).

6. Ajzen, I. Nature and Operation of Attitudes. Annu. Rev. Psychol. 52, 27-58 (2001).

7. Simon, H. A. \& Newell, A. Human problem solving: The state of the theory in 1970. Am. Psychol. 26, 145-159 (1971).

8. Higgins, E. T. Knowledge activation: Accessibility, applicability, and salience. in Social Psychology: Handbook of Basic Principles (eds. Higgins, E. T. \& Kruglanski, A. W.) 133-168 (Guilford Press, 1996).

9. Bordalo, P., Gennaioli, N. \& Shleifer, A. Salience Theory of Choice Under Risk. Q. J. Econ. 127, 1243-1285 (2012).

10. Koszegi, B. \& Szeidl, A. A Model of Focusing in Economic Choice. Q. J. Econ. 128, 53104 (2012).

11. Camerer, C. F., Loewenstein, G. \& Rabin, M. Advances in Behavioral Economics. (Princeton University Press, 2004).

12. Evans, J. S. B. T. \& Stanovich, K. E. Dual-process theories of higher cognition: Advancing the debate. Perspect. Psychol. Sci. 8, 223-241 (2013).

13. Fiske, S. \& Taylor, S. Social cognition: From brains to culture. (McGraw-Hill, 2013).

14. Simon, H. Theories of bounded rationality. in Decision and Organization 161-176 (1972).

15. Stahl, D. O. \& Wilson, P. W. On players' models of other players: Theory and experimental evidence. Games Econ. Behav. 10, 218-254 (1995).

16. Stanovich, K. E. The robot's rebellion: Finding meaning in the age of Darwin. (Chicago University Press, 2005).

17. Pennycook, G., Fugelsang, J. A. \& Koehler, D. J. What makes us think? A three-stage dual-process model of analytic engagement. Cogn. Psychol. 80, 34-72 (2015).

18. Lewandowsky, S., Ecker, U. K. H. \& Cook, J. Beyond Misinformation: Understanding and Coping with the "Post-Truth" Era. J. Appl. Res. Mem. Cogn. 6, 353-369 (2017).

19. Gallego, J., Martínez, J. D., Munger, K. \& Vásquez-Cortés, M. Tweeting for peace: Experimental evidence from the 2016 Colombian Plebiscite. Elect. Stud. 62, 102072 (2019). 
20. Desposato, S. Ethics and experiments: Problems and solutions for social scientists and policy professionals. (Routledge, 2015).

21. Taylor, S. J. \& Eckles, D. Randomized experiments to detect and estimate social influence in networks. in Complex Spreading Phenomena in Social Systems (eds. Lehmann, S. \& Ahn, Y. Y.) 289-322 (Springer, 2018).

22. Pennycook, G. \& Rand, D. G. Fighting misinformation on social media using crowdsourced judgments of news source quality. Proc. Natl. Acad. Sci. (2019). doi:10.1073/pnas.1806781116

23. Hodas, N. O. \& Lerman, K. The simple rules of social contagion. Sci. Rep. 4, 1-7 (2014). 